\title{
PUPILLARY LIGHT REFLEX DEFICITS IN A CANINE MODEL OF NEURONAL CEROID LIPOFUSCINOSIS AND THE EFFECTS OF ENZYME REPLACEMENT THERAPY
}

A Dissertation
presented to
the Faculty of the Graduate School
at the University of Missouri
In Partial Fulfillment
of the Requirements for the Degree
Doctor of Philosophy
beBECCA E.H. WHITING
Drs. Kristina Narfström and Martin L. Katz, Dissertation Supervisors
MAY 2013


(C) Copyright by Rebecca Whiting 2013

All Rights Reserved 
The undersigned, appointed by the dean of the Graduate School, have examined the dissertation entitled

\section{PUPILLARY LIGHT REFLEX DEFICITS IN A CANINE MODEL OF NEURONAL CEROID LIPOFUSCINOSIS AND THE EFFECTS OF ENZYME REPLACEMENT THERAPY}

presented by Rebecca E.H. Whiting,

a candidate for the degree of doctor of philosophy,

and hereby certify that, in their opinion, it is worthy of acceptance.

Dr. Kristina Narfström, Biological Engineering and Veterinary Medicine \& Surgery

Dr. Martin L. Katz, Ophthalmology

Dr. Gang Yao, Biological Engineering

Dr. Jinglu Tan, Biological Engineering

Dr. Joan R. Coates, Veterinary Medicine \& Surgery 
For my brother, Bryan. 


\section{ACKNOWLEDGEMENTS}

This project has required extensive collaboration, and I truly appreciate all of the people that have contributed to its successful completion. I thank my advisors, Drs. Kristina Narfström and Martin Katz, for their continued guidance and support throughout my studies, the resources they have contributed, and the time they have devoted to these projects. I have learned immensely under their supervision, and I will always deeply appreciate their contributions to my development as a scientist.

The contributions of Dr. Gang Yao and members of his lab, Xiaofei Fan and Chathuri Daluwatte, were essential in the development of the pupillography system. Dr. Yao has also been a great source of knowledge, guidance and encouragement throughout my studies. I would like to thank Dr. Joan Coates for her participation and patience in my education of veterinary medicine and for her input in the development and execution of this project. I am grateful to Dr. Jacqueline Pearce for her contributions to the development of the PLR protocol, for her continued support in visual assessment of the dogs, and for her encouragement during my studies. I thank Dr. John Dodam for his much needed assistance in the development of an anesthesia protocol for these experiments. I also appreciate input from Dr. Jinglu Tan in discussions for development of these projects and his contributions to my education.

Members of the comparative neurology lab provided extensive support in the completion of these experiments. I cannot thank Leilani Castaner enough 
for the veterinary support, the problem solving discussions, and the numerous hours expended in PLR recording sessions. I also thank Drs. Camille Flournoy, Christine Sibigtroth, and Melissa Carpentier for their veterinary expertise and assistance with anesthesia during PLR and ERG recordings. I am very grateful to Molly Williams for her assistance with image processing and to all of the students that assist in the lab every day. 


\section{TABLE OF CONTENTS}

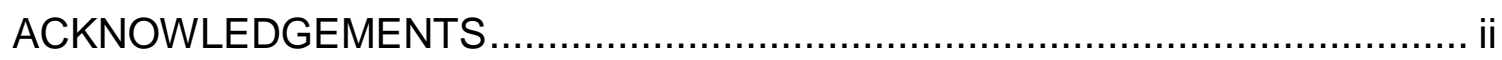

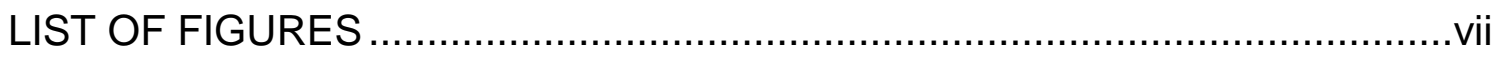

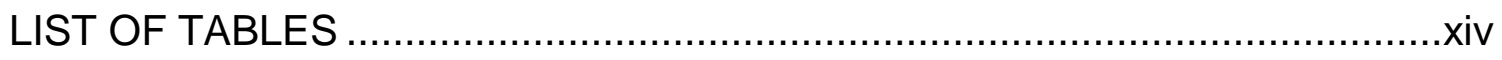

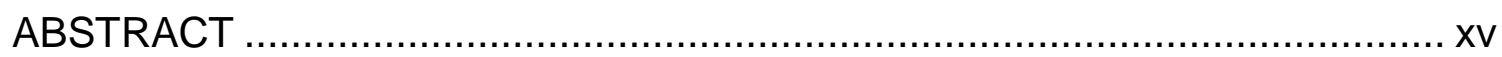

Chapter

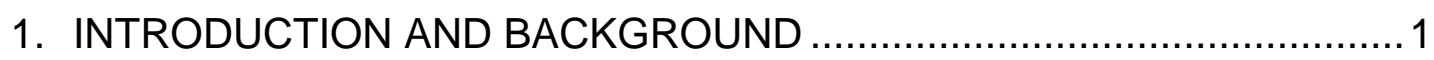

1.1. The Pupillary Light Reflex.......................................................

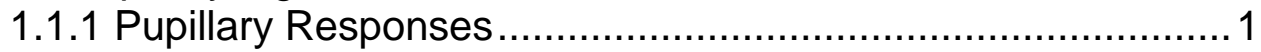

1.1.2 Retinal Input to the PLR ..............................................

1.1.3 PLR Neural Pathway ................................................. 4

1.2.Applications of PLR Quantification ..........................................

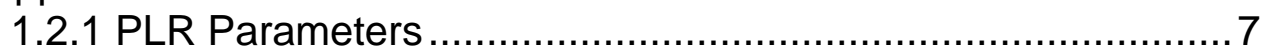

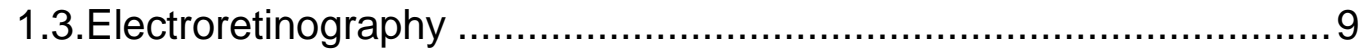

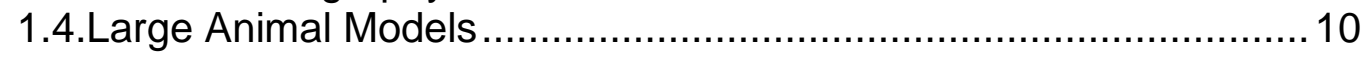

1.4.1 Canine Models.............................................................. 11

1.4.2 Differences in the Human and Canine Visual Systems ........12

1.5.Neuronal Ceroid Lipofuscinosis ................................................. 13

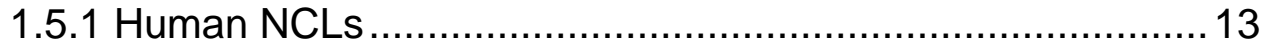

1.5.2 Canine Model of CLN2 …............................................. 14

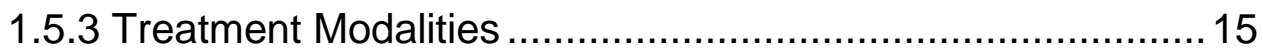

1.6.Study Objectives............................................................... 18

2. INSTRUMENTATION AND RECORDING METHODS

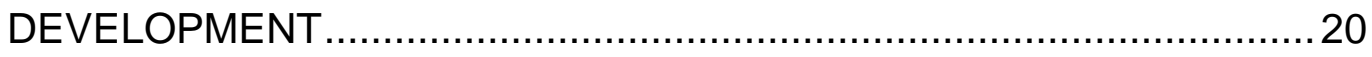

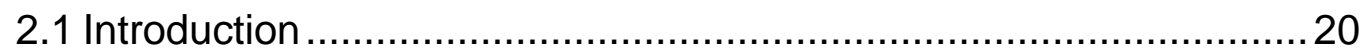

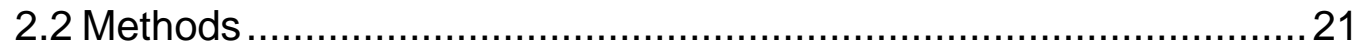

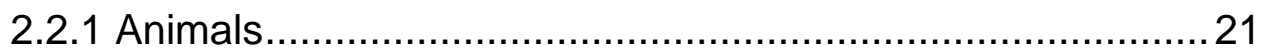

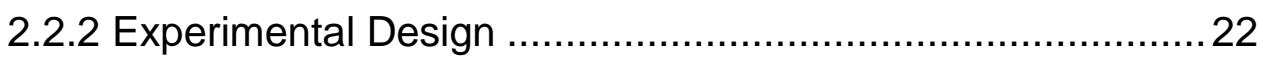

2.2.3 Chemical Restraint for PLR Recording ..............................22

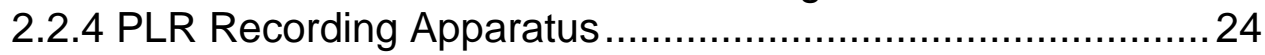

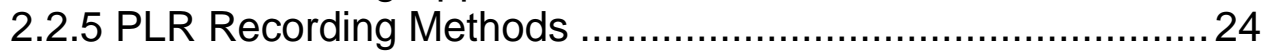

2.2.6 PLR Stimulus Protocols ..................................................26

2.2.7 Baseline Pupil Measurements .........................................2.

2.2.8 PLR Image Analysis .......................................................2

2.2.9 Chemical Restraint for Electroretinography .........................28 


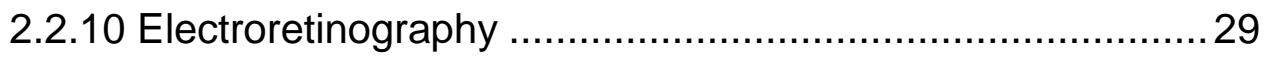

2.2.11 ERG Dog Diagnostic Protocol .......................................30

2.2.12 ERG Scotopic Intensity Series...................................... 32

2.2.13 Visually Evoked Potentials ............................................ 34

2.2.14 ERG and VEP Waveform Analysis ................................. 34

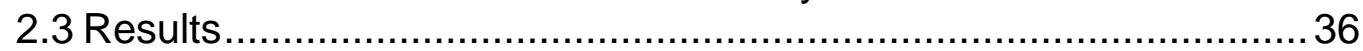

2.3.1 Effects of Sedation and General Anesthesia on the PLR .....36

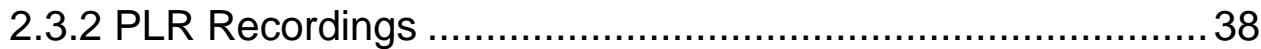

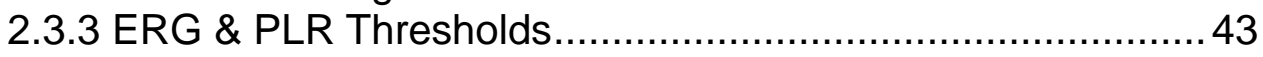

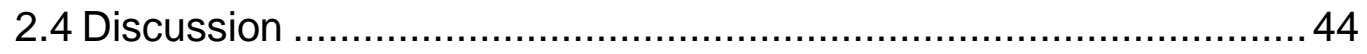

3. PLR DEFICITS IN A CANINE MODEL OF CLN2 …........................... 50

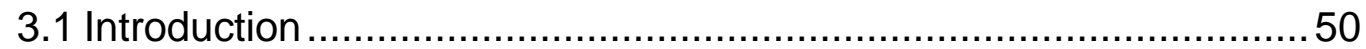

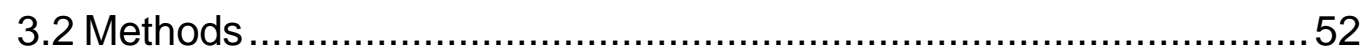

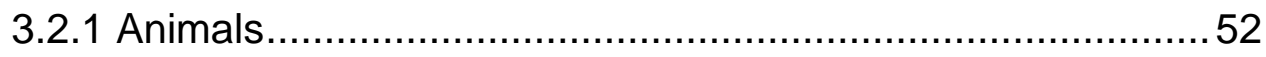

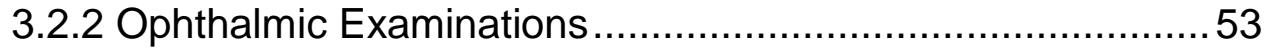

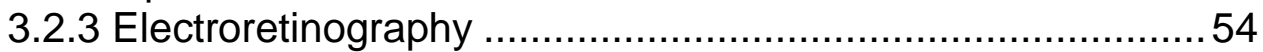

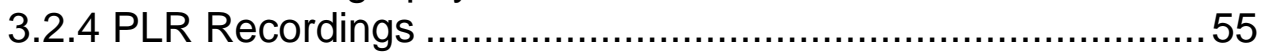

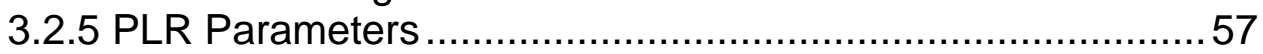

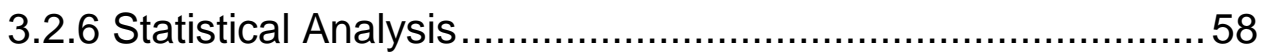

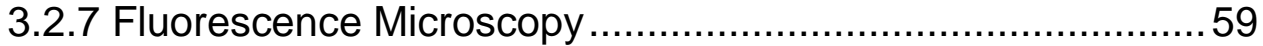

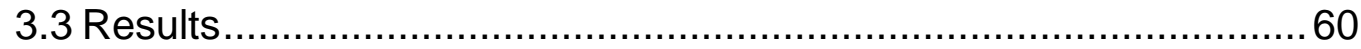

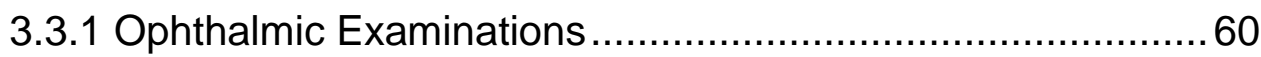

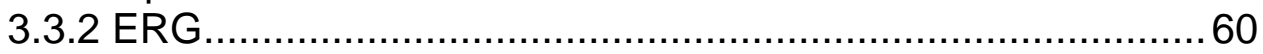

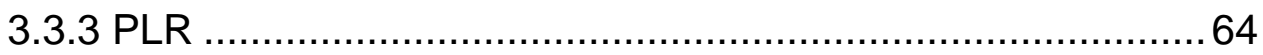

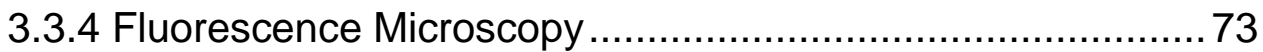

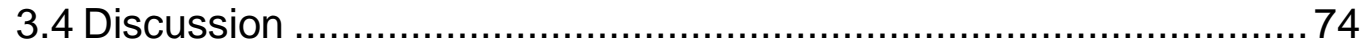

4. ENZYME REPLACEMENT THERAPY DELAYS CLN2-RELATED

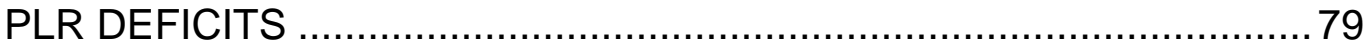

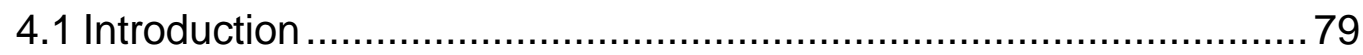

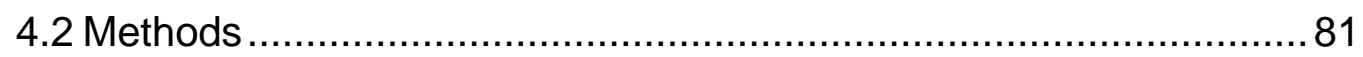

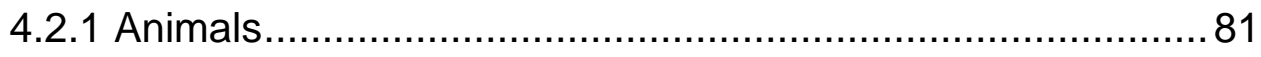

4.2.2 Delivery of Replacement Enzyme..................................... 81

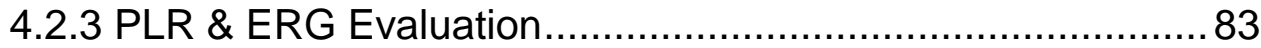

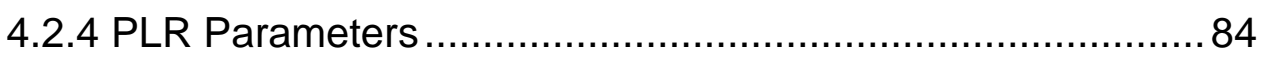

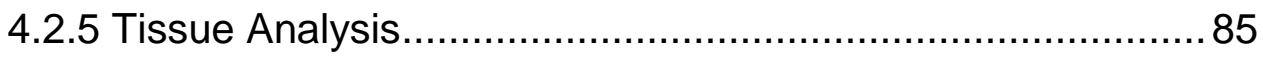

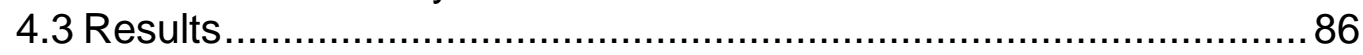

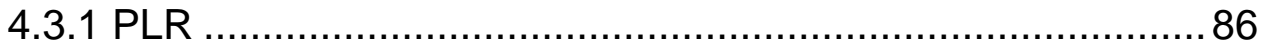

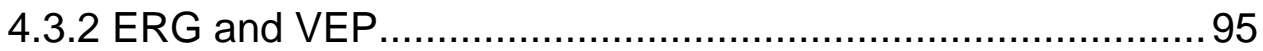

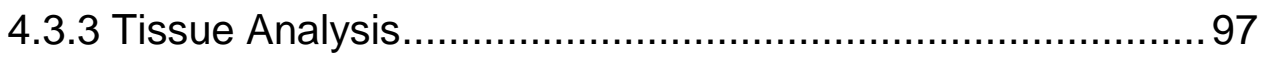

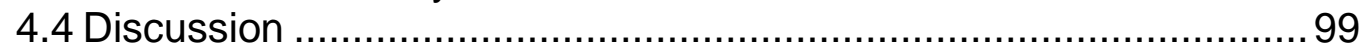


Appendix

A. PARTS LIST FOR THE PLR RECORDING SYSTEM ...................... 106

B. PHOTOSHOP IMAGE ANALYSIS “ACTION” STEPS ........................ 107

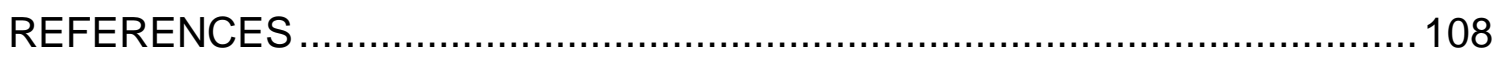

VITA 


\section{LIST OF FIGURES}

Figure

Page

1-1: Autonomic control of pupil size through innervation of the iris muscles. .....2

1-2: Parasympathetic pathway of the pupilliary light reflex. Input originates in the light sensitive elements of the retina, projects bilaterally to the pre-tectal nuclei, which each project bilaterally to the parasympathetic division of the oculomotor nuclei. Fibers project in the oculomotor nerve to the ciliary ganglion, and short ciliary nerves innervate the iris muscles. Modified figure from Dr. Dennis O’Brien.

1-3: Pathway for sympathetic input to the PLR in the dog.

1-4: Pupillogram showing the characteristic change in pupil area after a 100 millisecond stimulus. PLR parameters of interest can be calculated as indicated.

1-5: Characteristic ERG waveform components. The a-wave is the early negative component of the ERG and reflects activity of the retinal photoreceptor cells. The positive electrical potential of the ERG is called the b-wave, which reflects activity of cells in the inner retina. ...10

2-1: The camera and stimulus delivery portion of the PLR recording apparatus (left) and its schematic diagram (right). Photograph was taken in normal room light after the procedure.

2-2: Photoshop analysis showing selection of the pupil (red hash marks) based on pixel color (A). After expanding and contracting the selection by equal amounts, the hole in the selection caused by the infrared reflection is removed leaving an accurate outline of the pupil (B). 
2-3: ERG waveform analysis. The a-wave amplitude is measured as from the baseline to the trough of the a-wave, and the b-wave amplitude is measured from the trough of the a-wave to the peak of the b-wave. The a-wave and b-wave implicit times are measured as the time from onset of the flash stimulus to the trough of the a-wave or the peak of the b-wave, respectively.

2-4: VEP waveform analysis. The amplitude was measured for the peak occurring between 40 and 50 milliseconds after the flash. Implicit time was measured as the actual time from the flash to the measured peak.

2-5: Baseline pupil size with different chemical restraint protocols; dexmedetomidine and ketamine $(A)$ and dexmedetomidine and butorphanol (B). PLR traces from a dog under high-dose dexmedetomidine sedation $(C)$ and a dog under general anesthesia (D). Pupil responses are shown as percent of baseline pupil area. No stimulus was used in traces (A) and (B). A 100 millisecond flash stimulus of 11 log photons $/ \mathrm{cm}^{2} / \mathrm{s}$ was given 5 seconds into $(C)$ and $(D)$ recordings (dashed line) resulting in a large constriction. The substantial spontaneous fluctuation in pupil size observed under injectable chemical restraint $(A-C)$ was completely eliminated when the dog was anesthetized (D).

2-6: Baseline pupil behavior with different chemical restraint protocols. The dashed trace shows the fluctuating pupil size from a dog under dexmedetomidine sedation. The solid traces are from the same dog 3, 7, and 10 minutes after isoflurane anesthesia was initiated and show the gradual progression to a stable baseline pupil.

2-7: PLR traces illustrating the range of pupil constriction o,jbserved in anesthetized dogs with 100 millisecond white light stimuli of increasing intensity (from top to bottom: 8, 8.5, 9, 13, and 15 $\log$ photons $/ \mathrm{cm}^{2} / \mathrm{s}$ ). Pupil responses are shown as percent of baseline pupil area. The stimulus onset occurred 5 seconds into each recording. 
2-8: Blue and red traces show PLRs to 1 second stimuli of the corresponding color (top, 13 and $13.5 \mathrm{log}$ photons $/ \mathrm{cm}^{2} / \mathrm{s}$; bottom, 14.5 and $15 \mathrm{log}$ photons $/ \mathrm{cm}^{2} / \mathrm{s}$ ). The onset of each stimulus occurred 1 second into each recording. With stimuli of 13 and 13.5 log photons $/ \mathrm{cm}^{2} / \mathrm{s}$, the PLRs to red and blue light are very similar. The brighter blue stimulus (14.5 log photons $/ \mathrm{cm}^{2} / \mathrm{s}$ ) results in sustained pupil constriction, while the pupil quickly redilates after a red stimulus of comparable intensity. Recordings were performed in anesthetized dogs.

2-9: Average PLR constriction amplitude and PIPR $(n=7)$ and ERG $a$ - and $b$-wave amplitudes $(n=5)$ in response to white light stimuli of increasing intensity. Assuming a threshold for reliable detection of the PLR at 5\% pupil constriction and for the ERG b-wave an amplitude of $10 \mu \mathrm{V}$, the ERG threshold is more than 10 times higher than the PLR threshold. Pupil constriction amplitude is shown as percent of baseline pupil area. ERGs were recorded under dexmedetomidine sedation and PLRs were recorded under anesthesia with dexmedetomidine and isoflurane. Error bars represent standard error of the mean (SEM).

3-1: Representative ERG \& VEP tracings from a 10 month old normal dog (left), 6 month old affected dog (middle) and 10 month old affected dog (right). (A) Scotopic rod recordings and $10 \mathrm{~cd} . \mathrm{s} / \mathrm{m}^{2}$ mixed rod and cone recordings. (B) Photopic cone single flash and $30 \mathrm{~Hz}$ flicker recordings. (C) VEP recordings performed on light-adapted dogs.

3-2: ERG amplitudes as a function of age in normal and CLN2affected dogs. ERG a-wave amplitude from $10 \mathrm{~cd} . \mathrm{s} / \mathrm{m}^{2}$ scotopic (A) and photopic (B) recordings. Significant deficits in a-wave amplitude are not present until 10 months of age and only with scotopic recordings. ERG b-wave amplitude from scotopic rod responses (C), scotopic mixed responses from rods and cones at $10 \mathrm{~cd} . \mathrm{s} / \mathrm{m}^{2}(\mathrm{D})$, and photopic cone $(E)$ and $30 \mathrm{~Hz}$ flicker recordings $(F)$. Significant deficits in b-wave amplitude exist even in early stages of disease $\left(\dagger, p<0.001 ;{ }^{*}, p<0.005 ; \ddagger, p<0.05\right)$. Error bars represent SEM. 
3-3: VEP amplitude as a function of age in normal and CLN2-affected dogs. Results were variable with no statistical differences between normal and affected dogs. Data at 11 months of age reflects a single affected dog.

3-4: Baseline pupil diameter in anesthetized normal and CLN2-affected Dachshunds between 4 and 10 months of age. Baseline diameter is significantly reduced in 10 month old affected dogs $\left({ }^{*}, p<0.01\right)$.

3-5: Representative PLR traces from a normal dog at 10 months of age and a CLN2-affected dog between 4 and 10 months of age. Pupil responses are shown as percent of baseline pupil area. PLRs are in response to stimuli of $9,10,11$, and $15 \log$ photons $/ \mathrm{cm}^{2} / \mathrm{s}$ (A-D, respectively).

3-6: PLR constriction amplitude as a function of stimulus intensity in normal and CLN2-affected dogs. Amplitudes from normal dogs include combined data from dogs of all ages examined. For stimulus intensities between 9 and $11 \log$ photons $/ \mathrm{cm}^{2} / \mathrm{s}$, constriction amplitude of affected dogs was progressively reduced from normal; differences were significant beginning at 6 months of age $(\dagger, p<0.03)$. With a stimulus of $12 \mathrm{log}$ photons $/ \mathrm{cm}^{2} / \mathrm{s}$, constriction amplitude was not significantly reduced until 8 months of age $(\ddagger, p<0.02)$. Age was not a significant factor for stimuli of $8.5,8.75$, and $13 \log$ photons $/ \mathrm{cm}^{2} / \mathrm{s}$, but constriction amplitude of the CLN2-affected group as a whole was significantly reduced from normal $\left({ }^{*}, p<0.02\right)$. Error bars represent SEM.

3-7: PLR latency as a function of stimulus intensity. Latency is significantly longer in CLN2-affected dogs at 10 months of age for stimuli of $8.75,9$, and 11 log photons $/ \mathrm{cm}^{2} / \mathrm{s}$ $\left({ }^{*}, p<0.01\right)$ and by 8 months for a stimulus of $10 \mathrm{log}$ photons $/ \mathrm{cm}^{2} / \mathrm{s}(\dagger, p<0.02)$. In addition, the increase in PLR latency was significantly greater in the 10 month old CLN2affected dogs compared with 4 and 6 month old dogs for stimuli of 9 and $10 \log$ photons $/ \mathrm{cm}^{2} / \mathrm{s}$. Error bars represent SEM.

3-8: Average constriction velocity was significantly reduced in 10 month old CLN2-affected dogs with stimuli between 8.75 and $11 \log$ photons $/ \mathrm{cm}^{2} / \mathrm{s}\left({ }^{*}, \mathrm{p}<0.001\right)$. Error bars represent SEM 
3-9: Average redilation velocity as a function of stimulus intensity. Redilation velocity is significantly reduced in 10 month old CLN2-affected dogs with stimuli between 8.75 and $11 \mathrm{log}$ photons $/ \mathrm{cm}^{2} / \mathrm{s}\left({ }^{*}, p<0.005\right)$. Error bars represent SEM.

3-10: The secondary redilation phase is delayed in CLN2-affected dogs at 8 and 10 months of age. (A) Sample PLR traces from 10 month old dogs shown as percent of baseline pupil area. The normal dog pupil gradually redilates to its baseline size while the pupil from the affected dog maintains its constricted size for 85 seconds after light offset. (B) The rate of secondary redilation was decreased in older, affected dogs $\left({ }^{*}, p<0.025\right)$ indicating that constriction persists longer after light offset.

3-11: Fluorescence micrographs of unstained cryostat sections of pretectal neurons $(A, B)$ and the parasympathetic neurons of the oculomotor nucleus $(C, D)$ from 10.5 month old normal $(A, C)$ and $C L N 2$-affected $(B, D)$ Dachshunds. Yellow-emitting autofluorescent storage material was present in both brain regions of the CLN2-affected dogs.

4-1: Cellular transport of TPP1. TPP1 can be synthesized and transported to the lysosome for use within a single cell, or it can be excreted and taken up by a neighboring cell. With ERT, this results in the uptake and processing of infused TPP1 through normal cellular mechanisms.

4-2: Representative PLR traces from a normal dog, untreated CLN2affected dog, and an affected dog that received ERT between 8 and 15 months of age. Pupil responses are shown as percent of baseline pupil area. PLRs are in response to a stimulus of $10 \log$ photons $/ \mathrm{cm}^{2} / \mathrm{s}$.

4-3: PLR constriction amplitudes from 8 month old dogs. (A) Data from all CLN2-affected dogs treated with TPP1 enzyme replacement therapy is combined. While untreated dogs have significant deficits compared to normal $(\#, p<0.02)$, treated dogs are not significantly different from normal or untreated dogs. (B) Treated data is broken into 2 groups of dogs with Group 1 having normal constriction amplitudes that are significantly different from those of untreated, CLN2affected dogs $\left({ }^{*}, p<0.001 ; \dagger, p<0.01\right)$. 
4-4: PLR constriction amplitudes from 10 month old dogs. (A) Data from all CLN2-affected dogs treated with TPP1 enzyme replacement therapy is combined. Untreated dogs $(\#, p<0.02)$ and treated dogs $(\dagger, p<0.025)$ have significant deficits compared to normal. (B) Treated data is broken into 2 groups of dogs with Group 1 having normal constriction amplitudes that are significantly different from those of untreated, CLN2affected dogs $\left({ }^{*}, p \leq 0.001 ; \ddagger, p<0.025\right)$.

4-5: PLR constriction amplitudes from 12 month old CLN2-affected dogs. Amplitudes are reduced from normal in both Group 1 and Group 2, though Group 1 amplitudes are still significantly greater $(\ddagger, p<0.05)$ than those of Group 2 for stimuli of 9 and $10 \log$ photons $/ \mathrm{cm}^{2} / \mathrm{s}$.

4-6: PLR constriction amplitude changes with age for rhTPP1 treated dogs from Group 1 (A) and Group 2 (B). Group 1 shows a delay in PLR constriction amplitude deficits, but at 15 months of age, the dogs from each group exhibit very similar results.

4-7: PLR parameters in normal and 10 month old treated and untreated CLN2-affected Dachshunds. Maximum constriction velocity (A), maximum redilation velocity $(B)$, and latency $(C)$ are all normalized in Group 1 of the treated dogs and are significantly different than untreated dogs with dim intensity stimuli $\left({ }^{*}, p<0.05\right) \ldots . .93$

4-8: The secondary redilation phase of the PLR is delayed in treated CLN2-affected dogs between 8 and 12 months of age. The rate of secondary redilation was significantly decreased $\left({ }^{*}, p<0.05\right)$ indicating that constriction persists longer after light offset in these dogs than in normal dogs.

4-9: $E R G$ a-wave $(A, B)$ and b-wave $(C-F)$ amplitudes as a function of age in normal and CLN2-affected Dachshunds. ERG deficits are similar in CLN2-affected dogs that were untreated and those that received TPP1 ERT. ERG a-wave amplitude from $10 \mathrm{~cd} . \mathrm{s} / \mathrm{m}^{2}$ scotopic (A) and photopic (B) recordings. ERG b-wave amplitude from scotopic rod responses (C), scotopic mixed responses from rods and cones at $10 \mathrm{~cd} . \mathrm{s} / \mathrm{m}^{2}(\mathrm{D})$, photopic cone recordings (E), and photopic $30 \mathrm{~Hz}$ flicker recordings $(F)$. Treated dogs from Group 1 that exhibited normal PLR constriction amplitudes, exhibit no improvement in ERG b-wave amplitude. Error bars represent SEM. 
4-10: VEP amplitudes as a function of age. Results were variable and no differences were apparent amongst the treatment groups......

4-11: Fluorescence micrographs of unstained cryostat sections of the pretectal nucleus (left column; A,C,E) and the parasympathetic division of the oculomotor nucleus (right column; B,D,F) from normal dogs $(A, B)$, untreated CLN2-affected dogs $(C, D)$ and affected dogs treated with rhTPP1 (E, F). Yellow-emitting autofluorescent storage material was present in the neurons of both nuclei in the untreated CLN2-affected dogs (white arrows). While some storage material was present in the dogs treated with rhTPP1, it was much less prevalent and unlike the untreated dogs, there are neurons that do not appear to contain this material (red arrows). 


\section{LIST OF TABLES}

Table

Page

2-1: PLR stimulus protocol. All stimuli were presented as a 100 millisecond flash of white light beginning with the dimmest flash and gradually increasing as outlined in the table.

2-2: ERG dog diagnostic protocol. Photon flux values (log photons $/ \mathrm{cm}^{2} / \mathrm{s}$ ) were calculated from irradiance levels measured with a NIST-traceable spectroradiometer.

2-3: ERG scotopic intensity series protocol. ERG scotopic intensity series protocol. Photon flux values (log photons $/ \mathrm{cm}^{2} / \mathrm{s}$ ) were calculated from irradiance levels measured with a NIST-traceable spectroradiometer.

2-4: Baseline pupil fluctuation as a percent of average pupil size for the chemical restraint protocols tested. Range is the difference between the maximum and minimum pupil size over a 20 second baseline recording. Low-dose dexmedetomidine did not allow for reliable recordings making it impossible to quantify these measures.

2-5: PLR constriction amplitude shown as percent of baseline pupil area (average \pm SEM). Range is the difference between the maximum and minimum average constriction amplitude for the five ages tested.

2-6: PLR parameters as a function of stimulus intensity in anesthetized dogs. Values from all ages were averaged.

2-7: ERG a- and b-wave amplitudes from the scotopic intensity series 43

4-1: Experimental Treatment Groups 82 


\title{
PUPILLARY LIGHT REFLEX DEFICITS IN A CANINE MODEL OF NEURONAL CEROID LIPOFUSCINOSIS AND THE EFFECTS OF ENZYME REPLACEMENT THERAPY
}

\author{
Rebecca E.H. Whiting
}

Drs. Martin L. Katz \& Kristina Narfström, Dissertation Supervisors

\begin{abstract}
Pupil size is controlled by the autonomic nervous system, and iris behavior reflects a balance of input from both the sympathetic and parasympathetic nervous systems. The pupillary light reflex (PLR) occurs in response to light entering the eye and requires functional integrity of the retina and specific nuclei of the midbrain. Recently, pupillography or quantitative analysis of the PLR has been developed as a non-invasive, objective technique capable of detecting subtle changes associated with the complex network of neuronal circuitry involved in modulating pupil size. This makes the PLR a useful biomarker that can be used to monitor disease progression in neurological disorders.
\end{abstract}

The neuronal ceroid lipofuscinoses (NCLs) are a group of lysosomal storage disorders that are inherited in an autosomal recessive manner. A late-infantile onset form of NCL (CLN2) is caused by a mutation in the CLN2 gene which codes for tripeptidyl peptidase-1 (TPP1), a soluble, lysosomal enzyme that aids degradation of peptides in cells throughout the body. A Dachshund model of CLN2 was developed and is currently being maintained at the University of Missouri. Dogs affected by CLN2 lack functional TPP1 and present with 
progressive ataxia, cognitive and behavioral changes, and myoclonic seizures starting at approximately 7-8 months of age and progressing to a terminal state requiring euthanasia at 10 to 11 months of age. In addition, affected dogs exhibit vision loss and marked deficits in ERG b-wave amplitude and significant thinning of the inner retina by disease end-stage. The strong resemblance to the human CLN2 makes these dogs an excellent model in which to test possible treatment options prior to beginning human clinical trials.

In the effort to make optimal use of the canine model of CLN2, studies were undertaken to develop a reliable protocol for the quantitative assessment of the canine PLR. Using the developed equipment and methodology, we thoroughly evaluated the PLR in response to short flashes of white light of increasing intensity in normal and CLN2-affected dogs. We found that CLN2-affected dogs exhibit PLR deficits that progress with age and other symptoms of disease. Deficits are particularly apparent with dim stimuli and likely result from a combination of decline in retinal function and disease related changes in areas of the midbrain involved in modulating the PLR.

In the Dachshund CLN2 disease model, it was previously shownthat administration of recombinant TPP1 via infusion into the cerebrospinal fluid (CSF) results in widespread distribution and uptake of the active enzyme into many structures of the brain and in reduction in the accumulation of neuronal lysosomal storage material that is characteristic of this disease. Studies were undertaken to determine if TPP1 enzyme replacement therapy (ERT) could $x v i$ 
ameliorate PLR deficits associated with CLN2. One-third of the dogs treated with ERT exhibited a substantial delay in the appearance of PLR deficits compared with untreated, affected dogs. However, no improvements in retinal function could be measured by the ERG. It is unlikely that TPP1 reached the retina, and it is more likely that ERT normalized the PLR by preventing degeneration in areas of the midbrain involved in modulating the PLR. However, further analysis of retinal and midbrain tissues is necessary to fully explain these treatment effects.

The characterization of disease-related alterations in light induced responses contributes to our understanding of the pathology underlying CLN2. Preventing development of the deficits in the PLR and ERG responses can be used to objectively assess the efficacy of therapeutic interventions for CLN2 that are currently being evaluated. An ideal therapy would prevent the declines in both the PLR and the ERG responses by preventing both retina and brain degeneration. 


\section{CHAPTER 1}

\section{INTRODUCTION AND BACKGROUND}

\subsection{The Pupillary Light Reflex}

\subsubsection{Pupillary Responses}

The iris of the mammalian eye regulates the amount of light that reaches the retina by modulating the size of the opening at the center of the iris called the pupil. Pupil size is controlled by the autonomic nervous system, and therefore iris behavior reflects the function of both the sympathetic and parasympathetic nervous systems. The iris consists of two sets of antagonistic smooth muscles, one for constriction and the other for dilation (Figure 1-1). The parasympathetic system releases acetylcholine to both activate the circularly arranged sphincter muscles and to inhibit the muscles responsible for dilation allowing constriction to occur more rapidly and to a greater extent. The sympathetic system releases norepinephrine which activates the radially arranged dilator muscles and also inhibits the constrictor muscles (Yoshitomi and Ito 1986; Yoshitomi, Ito, and Inomata 1985). The size of the pupillary opening at any moment is determined by the balance between the sympathetic and parasympathetic input to the iris muscles. 


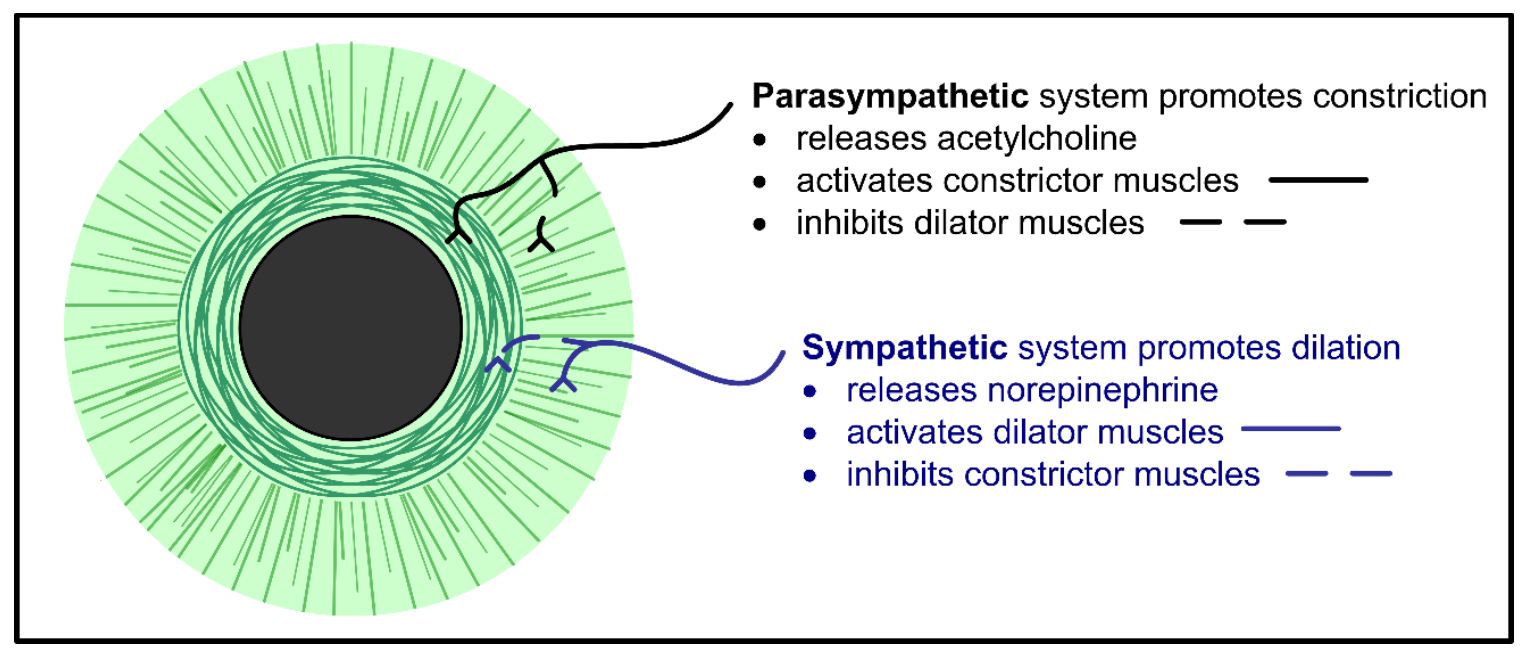

Figure 1-1: Autonomic control of pupil size through innervation of the iris muscles.

Pupil size changes in response to many types of stimuli including noise, pain, surprise, pleasure and stress which all cause pupil dilation from activation of the sympathetic nervous system (Bradley et al. 2008; Lowenstein and Lowenfeld 1950). Cognitive tasks can also cause pupil dilation through cortical inhibition of parasympathetic input to the iris muscles (Steinhauer et al. 2004). However, the most prominent factor regulating pupil size is the amount of light that enters the eye. The retina has a limited dynamic range. To help keep the amount of light reaching the retina within this range, pupil size is reduced under bright light conditions and is increased under dim light conditions. These changes in pupil size in response to alterations in light exposure are called the pupillary light reflex (PLR).

Mammals have both a direct PLR of the stimulated eye, and a consensual PLR of the contralateral, unstimulated eye (Scagliotti 1999). The dynamics of the 
PLR vary depending on the characteristics of the light stimulus, such as intensity, duration, and color. The PLR is also known to change with conditions that affect the autonomic nervous system including disease and trauma. This makes the PLR a non-invasive biomarker that can be used to monitor neurological status.

\subsubsection{Retinal Input to the PLR}

The signal to initiate the PLR begins in the light sensitive elements of the retina, consisting of rod and cone photoreceptors and intrinsically photosensitive retinal ganglion cells (ipRGCs) (Markwell, Feigl, and Zele 2010; Gamlin et al. 2007; Dacey et al. 2005a). The ipRGCs contain the photopigment melanopsin, which allows them to independently collect information about overall light levels in the environment and project to centers of the brain responsible for the PLR and circadian photoentrainment (Brown and Lucas 2009). All rod and cone input to the PLR is routed through the ipRGCs where input from all three cell types is combined and transmitted to the areas of the midbrain responsible for the PLR (Ecker et al. 2008). Genetically modified mouse models have demonstrated that rods, cones, and ipRGCs are all essential to a fully normal PLR (Lucas et al. 2003; Hattar et al. 2003). In mice lacking the melanopsin photopigment but with intact ipRGC neural connections, the pupil is unable to fully constrict at high irradiances. Loss of the rod and cone system results in an increased threshold and reduced constriction amplitude with all stimuli except those of very high intensity (above 14 log photons $/ \mathrm{cm}^{2} / \mathrm{s}$ ) (Lucas et al. 2003). When ipRGCs are ablated, animals lose non-image forming visual function including the PLR and 
circadian entrainment. However, since this neural pathway is separate from that required for image forming vision, animals lacking ipRGCs retain pattern vision (Ecker et al. 2008).

\subsubsection{PLR Neural Pathway}

The pathway for parasympathetic input of the PLR (Figure 1-2) originates in the light-sensitive elements of the retina, including rods, cones, and intrinsically photosensitive melanopsin ganglion cells (Gamlin et al. 2007; Markwell, Feigl, and Zele 2010). The signal from the retina is conveyed in the optic nerve through the optic chiasm and optic tract. Axons in the optic tract not involved with vision diverge from the visual pathway at the lateral geniculate nucleus to the pretectal nuclei at the level of the midbrain. The majority of axons of the pretectal nuclei cross to synapse on the contralateral neurons of the oculomotor nerve nuclei, which provide the parasympathetic innervations to the pupil. Some axons of the pretectal nuclei remain ipsilateral. Axons from the parasympathetic nuclei course in the oculomotor nerve and synapse in the ciliary ganglion. Postganglionic neurons from the ciliary ganglion innervate the constrictor muscle of the pupil via the short ciliary nerve. Stimulation results in constriction of the pupil. There is bilateral input to the pretectal nuclei and therefore bilateral projections to the autonomic division of the oculomotor nuclei. Thus, light directed into one eye results in constriction of both pupils. In addition, the parasympathetic system can be centrally inhibited at the parasympathetic 
division of the oculomotor nucleus (Dahlstrom et al. 1964; Lawrence G. Sharpe and Pickworth 1981).

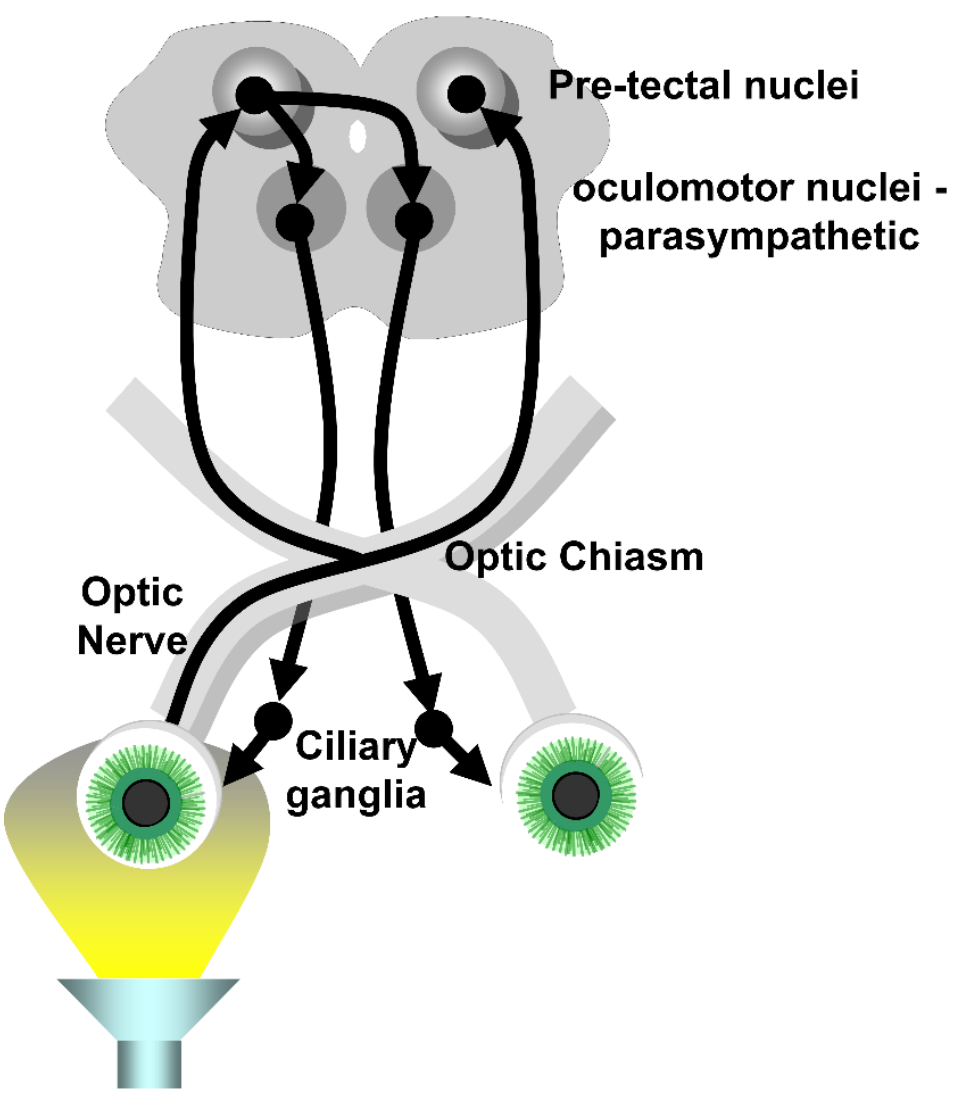

Figure 1-2: Parasympathetic pathway of the pupillary light reflex. Input originates in the light sensitive elements of the retina, projects bilaterally to the pretectal nuclei, which each project bilaterally to the parasympathetic division of the oculomotor nuclei. Fibers project in the oculomotor nerve to the ciliary ganglion, and short ciliary nerves innervate the iris muscles. Modified figure from Dr. Dennis O’Brien.

Pathways for sympathetic input to the PLR (Figure 1-3) originate in the hypothalamus and axons course ipsilaterally down the brainstem and cervical spinal cord via the lateral tectotegmental tracts to synapse on lower motor 
neurons located in the intermediate gray matter of the spinal cord segments T1 through T3 (Scagliotti 1999). Preganglionic (first order neurons) fibers leave the spinal cord and project as the cranial sympathetic trunk, which courses cranially in the neck synapsing in the cranial cervical ganglion. Finally, postganglionic sympathetic axons (second order neurons) then follow other cranial nerves to innervate structures in the head. Sympathetic nerves innervate smooth muscle in the periorbital facia, other structures around the eye and the dilator muscles of the iris.

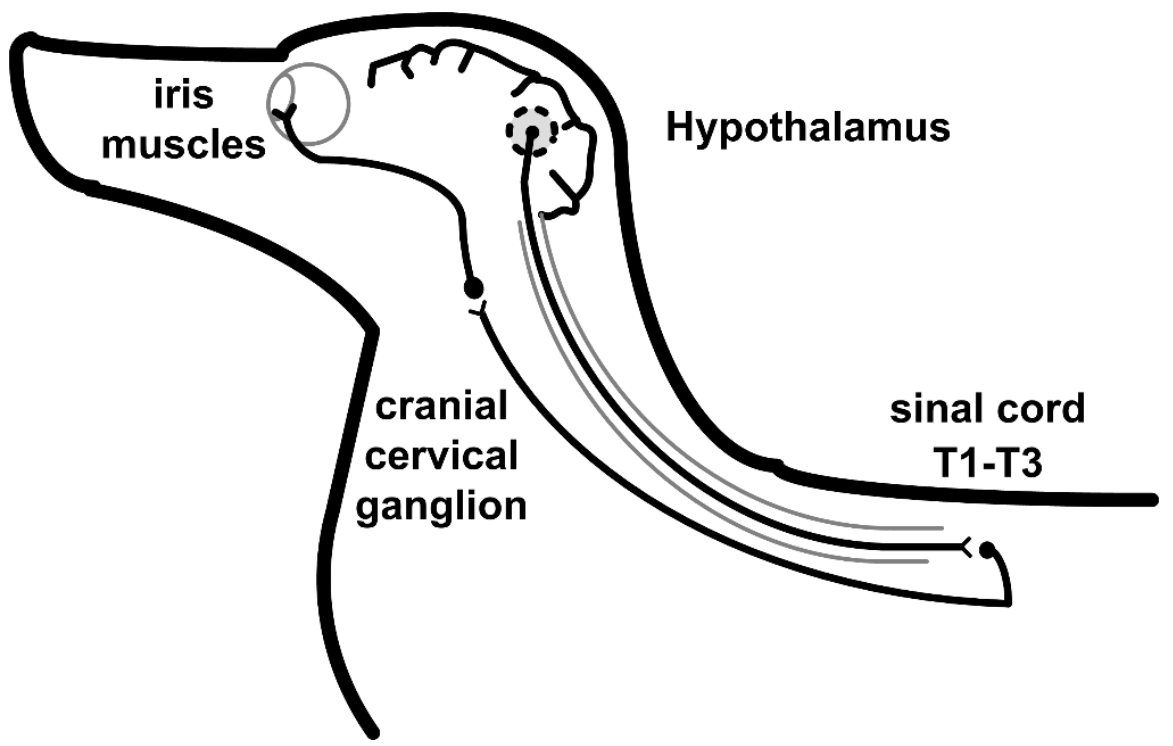

Figure 1-3: Pathway for sympathetic input to the PLR in the dog.

Axons of both sympathetic and parasympathetic nerves enter the periorbital region through the orbital fissure and travel through the choroid to the iris. Pupil size is ultimately determined by the balance of input from these two systems (Lowenstein and Lowenfeld 1950). 


\subsection{Applications of PLR Quantification}

Assessment of the pupillary light reflex (PLR) has long been used to assist in disease diagnosis for both human and veterinary medicine (Wilhelm 2008; Bremner 2009). However, classical methods of evaluating pupil abnormalities are largely qualitative and can only be used as a discriminating measure when substantial deficits are present. Recently, pupillography or quantitative analysis of the PLR has been developed as a non-invasive, objective technique capable of detecting subtle changes associated with the complex network of neuronal circuitry involved in modulating pupil size (Park et al. 2011; F. Fotiou et al. 2000). In addition to its use in the study of retinal disease (Thompson et al. 2010; Grozdanic et al. 2007; Melillo et al. 2012), pupillography has been used to detect autonomic dysfunction in neurologic disorders including multiple sclerosis (De Seze et al. 2001), Alzheimer's disease, Parkinson's disease (Fotiou et al. 2009), and autism spectrum disorders (Fan et al. 2009a). Quantification of specific PLR parameters (Figure 1-4) in these studies allowed for the analysis of small but real changes that may not have been detectable upon gross observation.

\subsubsection{PLR Parameters}

The PLR parameters that are typically considered are illustrated in Figure 1-4. For the studies described here, baseline pupil diameter is the average pupil diameter in a dark-adapted dog, over a 1-second period before the light stimulus. PLR constriction amplitude is defined as the difference between baseline pupil diameter and minimum pupil diameter attained following a light stimulus. Latency 
is defined as the time between stimulus onset and the beginning of pupil constriction. Average constriction velocity is calculated as the constriction amplitude divided by the constriction time, where constriction time is calculated between the beginning of pupil constriction and the minimum pupil diameter. Redilation of the pupil is biphasic with a fast initial redilation and slower secondary redilation. Average redilation velocity is defined as half the constriction amplitude divided by the time required for the pupil to redilate from its minimum diameter to half the baseline pupil size. The post illumination pupil response (PIPR) is calculated as the difference between baseline pupil diameter and sustained pupil diameter, or the average diameter for a defined period during the secondary redilation phase.

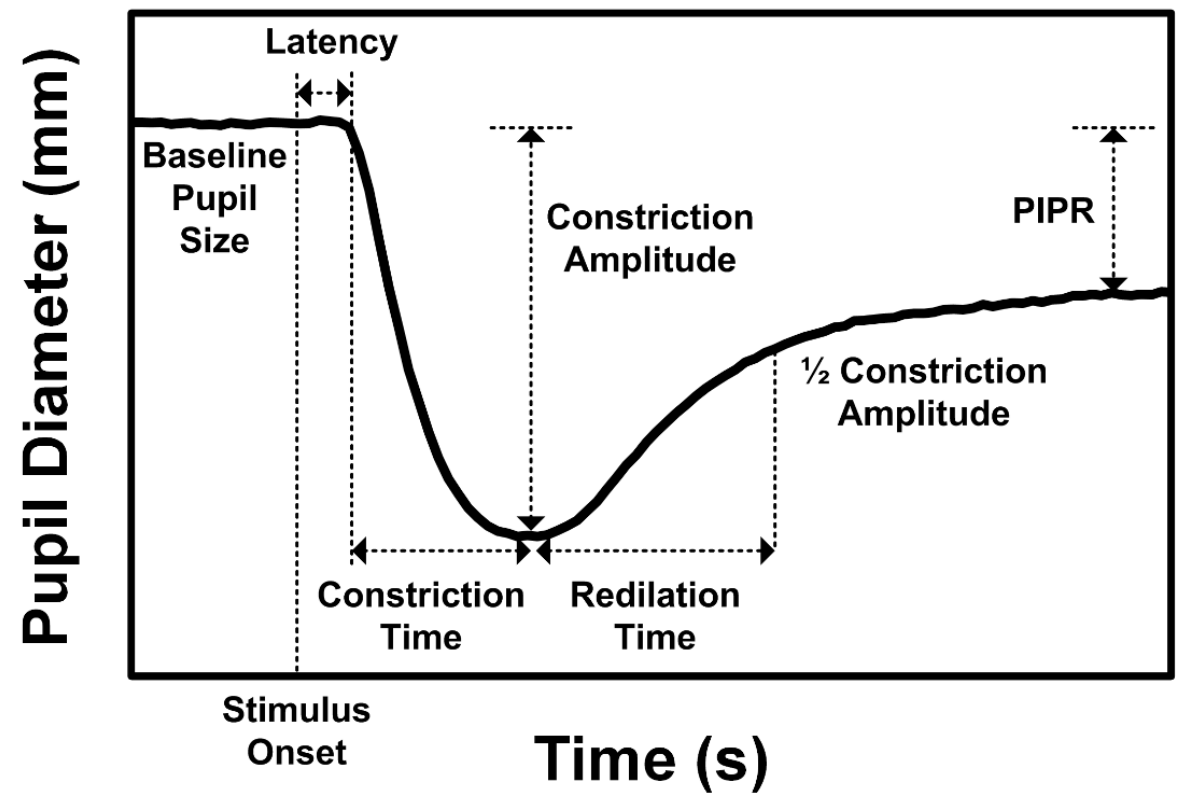

Figure 1-4: Pupillogram showing the characteristic change in pupil area after a 100 millisecond stimulus. PLR parameters of interest can be calculated as indicated. 


\subsection{Electroretinography}

The electroretinogram (ERG) is a recording of the electrical potential generated by the retina in response to a light stimulus. This potential can be detected non-invasively by placing a contact electrode on the cornea and a reference electrode on or under the nearby skin. Since the generated potential reflects the function of underlying cells, it is possible to monitor the amplitude and timing of this response for abnormalities as a measure of functional integrity of the cells. For this reason, the ERG is commonly used as a diagnostic tool in human and animal cases of retinal degeneration.

The ERG waveform has characteristic components depending on the parameters of the light stimulus. The early negative component of the ERG is called the a-wave (Figure 1-5) and reflects activity of the retinal photoreceptor cells (Penn and Hagins 1969). When a photon of light is absorbed in the photoreceptor outer segments, a transformational change occurs in the opsin molecules, or photopigments, which initiates a cascade to close membranebound ion channels. This in turn decreases ion flux and causes the cell to hyperpolarize (Hargrave and McDowell 1992; Yau 1994). This cascade occurs simultaneously in thousands of opsin molecules per photoreceptor cell. Since photoreceptors are arranged in parallel in the eye, the extracellular currents sum together and can be recorded externally from the cornea. When photoreceptor cells hyperpolarize they cease the synaptic release of neurotransmitter to second order neurons, which causes these bipolar cells to depolarize. The resulting 
current can be recorded as the positive electrical potential of the ERG called the b-wave (Figure 1-5) (Green and Kapousta-Bruneau 1999; Gurevich and Slaughter 1993; Stockton and Slaughter 1989). Increasing the intensity of the light stimulus results in a larger number of photons absorbed by the photoreceptors and a larger number of transmissions to bipolar cells. This results in larger summed currents from each cell layer, which is reflected in the ERG by an increase in amplitude of the corresponding waveform components.

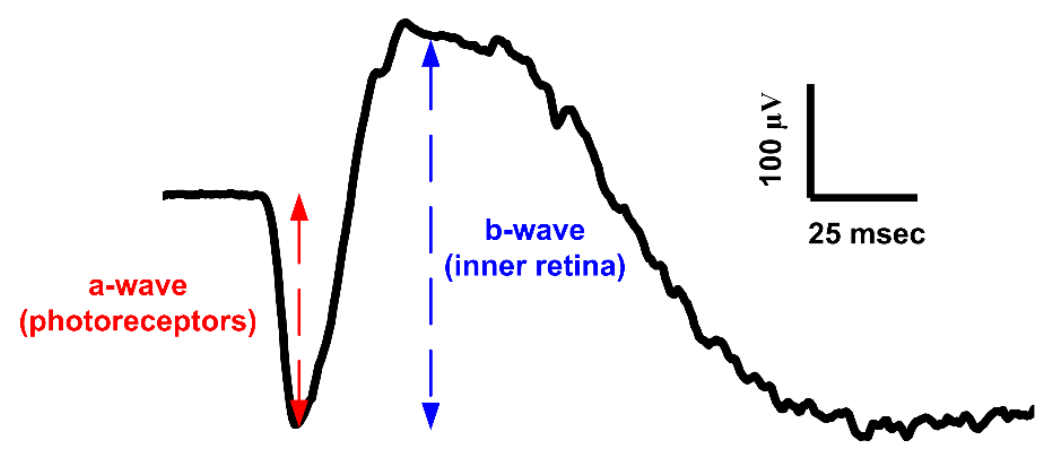

Figure 1-5: Characteristic ERG waveform components. The a-wave is the early negative component of the ERG and reflects activity of the retinal photoreceptor cells. The positive electrical potential of the ERG is called the b-wave, which reflects activity of cells in the inner retina.

\subsection{Large Animal Models}

Animal models of human disease play an important role in the understanding of disease mechanisms and in the development of treatment methodologies. While mice have several advantages such as low cost, short reproductive time, large litter size, and the opportunity for genetic modification, they are limited in 
their ability to accurately approximate human disease. Large animals such as dogs are more genetically, anatomically, and physiologically similar to people and fill a critical role as models for human disorders. Large animal models also allow for longitudinal studies to determine long-term safety and efficacy of therapeutic interventions that are not possible in rodents. In addition, proper scaling of treatment is much easier and more accurate in large animals. While there is a 1000 -fold difference in size between the brain of a mouse and that of a newborn child, there is only a 10 -fold difference between a small dog and child (Casal and Haskins 2006).

\subsubsection{Canine Models}

The dog is the most widely studied large animal model for genetically inherited diseases; over 350 canine genetic diseases have been identified (Sargan 2004). A large majority of genetic diseases are true orthologues of human disease with mutations identified in the analogous gene in both dogs and people (Ostrander and Giniger 1997). Dogs have been invaluable for the understanding of disease pathogenesis for a variety of diseases including retinal degeneration (Veske et al. 1999; M. L. Katz et al. 2008), multiple lysosomal storage disorders (Bond et al. 2013; Ray et al. 1998), hemophilia (Herzog et al. 1999), immunodeficiency (Felsburg et al. 1998), and neoplasia (Bianco et al. 2003; Chauvet et al. 1998). Canine models for many of these diseases exist in research colonies which provides the opportunity for thorough characterization of the disease phenotype and evaluation of treatment approaches. 


\subsubsection{Differences in the Human and Canine Visual Systems}

Axons of the retinal ganglion cell neurons coalesce to form the optic disc and course in the optic nerve. The fibers of the visual pathway and PLR are both part of the optic nerve. At the optic chiasm, axons originating from the medial (nasal) retina decussate and continue as the contralateral optic tract. Axons from the lateral (temporal) retina remain ipsilateral (do not cross at chiasm) and course in the ipsilateral optic tract.

The degree of decussation at the optic chiasm varies in different species. As a general rule, the more lateral the eyes are located on the head, the greater the degree of decussation and the less vision is binocular. In primates, essentially all the fibers from the medial half of the retina cross, while all the fibers from the lateral half of the retina remain ipsilateral enabling binocular vision. In carnivores, all of the fibers from the medial retina decussate in addition to approximately half of the fibers of the lateral retina. In people, $50 \%$ of the optic nerve fibers cross at the optic chiasm, while $75 \%$ of fibers decussate in dogs. Mammals have both a direct PLR in the stimulated eye, and a consensual PLR in the contralateral, unstimulated eye. This is due to the decussation of fibers at the optic chiasm and a second decussation at the pretectal nuclei. The unequal decussation in dogs results in a contraction anisocoria, or a difference in pupil constriction between the stimulated eye (direct) and the contralateral, unstimulated eye (consensual) (Scagliotti 1999). This prominent anisocoria is not present in people since optic nerve fibers decussate evenly. 
Dogs and people also differ in spectral sensitivity due to a difference in retinal photopigments. While both human and canine retinas contain rod, cone, and ipRGC photopigments, these pigments differ in the wavelengths of light to which they are most sensitive. The human retina has a rod peak sensitivity of $500 \mathrm{~nm}$ and three types of cones with peak sensitivities at $564 \mathrm{~nm}, 533 \mathrm{~nm}$, and 437 nm (Bowmaker and Dartnall 1980; Roorda and Williams 1999). The dog has a rod peak sensitivity at $508 \mathrm{~nm}$ and two cones with peak sensitivities at $429 \mathrm{~nm}$ and 555nm (Neitz et al. 1989). Melanopsin has been shown to have peak sensitivity around $480 \mathrm{~nm}$ in people, non-human primates (Gamlin et al. 2007), and mice (Hattar et al. 2003) though it hasn't specifically been studied in the dog.

\subsection{Neuronal Ceroid Lipofuscinosis}

\subsubsection{Human NCLs}

The neuronal ceroid lipofuscinoses (NCLs) are a group of lysosomal storage disorders that are inherited in an autosomal recessive manner (Haltia and Goebel 2012). Currently, there are at least 14 different forms of NCL.

Historically, the NCLs were classified by age of onset and by the ultrastructure of the accumulated storage material in the lysosomes (Haltia 2006), but with the identification of the causative mutations, the NCLs are now being classified based on the identity of the gene in which the mutation resides (Mole et al. 2011). While symptoms do vary amongst the different forms of NCL, most cases manifest with cognitive and motor impairment, vision loss, seizures, and premature death. These symptoms invariably worsen with age as the disorders 
progress. Disease progression in NCL correlates with an increase in abnormal storage material and neuronal degeneration.

A late-infantile onset form of NCL (CLN2) is caused by a mutation in the CLN2 gene which codes for tripeptidyl peptidase-1 (TPP1), a soluble, lysosomal enzyme that aids degradation of peptides in cells throughout the body.

Specifically, TPP1 acts to cleave tripeptides at the N-terminal of larger peptides generated during lysosomal protein degradation (Tomkinson 1999). In normal cells, proteins are regularly degraded into their component amino acids by a succession of several peptidases, including TPP1. These free amino acids can then be used for new protein synthesis and metabolic purposes. Therefore, the role of TPP1 is quite important in maintaining the normal balance between synthesis and degradation within the cell. The absence of TPP1 in the brain and retina leads to accumulation of proteins typically degraded by the enzyme and degeneration of neurons, thereby causing the symptoms associated with CLN2.

\subsubsection{Canine Model of CLN2}

A Dachshund model of CLN2 was developed and is currently being maintained at the University of Missouri. Affected dogs are homozygous for a frameshift mutation in the TPP1 gene (the ortholog of the human CLN2 gene). The dogs have a single base pair deletion in exon 4 of the gene (of 13 exons total) that alters the reading frame of the messenger RNA and creates a premature stop codon. Consequently, the protein is not made properly and the activity of TPP1 in the brain is eliminated (Awano et al. 2006b). Analysis of the 
storage bodies present in the tissue of affected dogs revealed that they consist mainly of curvilinear forms (Awano et al. 2006b). This is consistent with the ultrastructure of storage bodies that accumulate in the human form of CLN2 (Haltia 2006). In addition, the dogs present with mental dullness and ataxia by

6.5 months of age and extreme vision loss and myoclonic seizures by 8 months of age (Katz et al. 2008). Euthanasia is typically necessary between 10 and 11 months of age due to the progression of neurologic signs. This strong resemblance to the human CLN2 makes these dogs an excellent disease model on which to test possible treatment options prior to beginning human clinical trials.

\subsubsection{Treatment Modalities}

Enzyme replacement therapy (ERT) has long been considered as a possible treatment for lysosomal storage disorders, and it has proved effective in treating human disease of the peripheral tissue (Beutler 2004). However, due to the blood-brain barrier, systemic delivery of a missing enzyme has not been successful for disorders involving the central nervous system (CNS). If the blood-brain barrier can be bypassed, then ERT may provide hope for treating CNS dysfunction seen in the NCLs. One option is to inject the enzyme directly into the ventricular system of the brain. Since the cerebrospinal fluid (CSF) circulates throughout the brain via the ventricular system and subarachnoid space, any injected enzyme should be distributed throughout the brain as well. The enzyme can then be taken up by cells through a process mediated by the 
surface receptor for mannose-6-phosphate. TPP1 is then transported by the endosomal system to the lysosome allowing it to restore normal function in protein degradation.

In a mouse model of late-infantile NCL, intraventricular enzyme replacement resulted in increased TPP1 activity throughout the brain (Chang et al. 2008). Treated mice exhibited a slowed progression of tremor development and a reduction in GFAP staining. GFAP is the principle component of astrocytic intermediate filaments. In the NCLs, over-expression of GFAP has been observed as a sign of astrocytic activation and gliosis that accompany neuronal degeneration (Xu et al. 2010). The presence of autofluorescent storage material was also reduced in the treated mice compared with untreated, affected mice. While these results are certainly promising, this study was limited by the fact that TPP1 was only delivered over the course of 2 weeks. Complications with glial scarring around the cannula resulted in seizures, which precluded the use of survival as a measure of outcome (Chang et al. 2008). The treatment clearly requires long term testing and more thorough evaluation of outcome in a larger animal model before human clinical trials can be performed. Therefore, the NCL Dachshund model is currently being used to investigate intraventricular / intrathecal delivery of the TPP1 enzyme and its ability to rescue the NCL phenotype.

Gene therapy may offer a treatment option using a principle similar to that of enzyme replacement therapy. Viral-mediated gene therapy exploits the ability of 
a virus to infect a cell and insert its DNA in the nucleus of that cell. In gene therapy employing viral vectors, the genome of the virus is replaced with a desired therapeutic gene. Adeno-associated viruses (AAV) are currently one of the most commonly used viruses for this purpose. Unlike some viral vectors, $A A V s$ are able to infect non-dividing cells such as neurons (Daya and Berns 2008). In AAV vectors used for gene therapy, the entire coding portion of the vial genome is removed, so there is no possibility of viral replication in transfected cells.

AAV-mediated gene therapy has been tested as a treatment in a mouse model of CLN2. Increased TPP1 activity was observed up to 18 months after injection, no adverse reactions were noted, and a decrease in accumulated storage material was observed (Sondhi et al. 2007). The mice also showed improvements in gait, seizure activity, and longevity. These findings prompted a human clinical trial to study the safety of gene therapy in treating CLN2. In this study patients received injections of an AAV-TPP1 vector into multiple locations within the brain parenchyma. This trial was not designed to test efficacy, so it was not matched, randomized, or blinded and lacked a placebo/sham group. It did, however, report an improvement in neurological performance (based on a clinical rating scale), while no improvement was seen in the MRI of the subjects (Worgall et al. 2008). These results were somewhat promising, but more recent studies have failed to detect a therapeutic benefit in children treated in this manner (Ron Crystal, personal communication). An alternative approach to 
AAV-TTP1 mediated gene therapy for the CNS would be to transfect the ependymal cells that line the ventricular system with the vector. With this approach, the TPP1 would be expected to be released into the CSF and then distributed throughout the brain via the CSF circulation. The canine model of NCL provides a unique opportunity to study these approaches to treatment before human testing. With the dog model, normal littermate controls $\left(T P P 1^{+/+}\right)$ and untreated affected dogs are commonly available for comparison with respect to treatment efficacy.

\subsection{Study Objectives}

Disease progression in the canine model of CLN2 has been characterized with respect to the accumulation of storage material, neurologic symptoms, behavioral and cognitive changes, and decline in retinal function. Quantification of the PLR may offer an additional biomarker for noninvasive, functional evaluation of neurons, both in the brain and the retina. In addition to testing treatment efficacy in a canine disease model of NCL, objective PLR measurements may provide a method of monitoring disease progression and treatment in human patients as well. Due to the non-invasive nature and flexibility of the PLR, it can be easily translated to monitor disease progression and treatment effects in human clinical trials. The successful demonstration of PLR as an objective measure of neuronal function in dogs will introduce a useful tool for assessing therapeutic responses, thus helping to advance the development of lifesaving treatments for children affected by NCL. 
The specific aims for this study are as follows:

1. Develop PLR recording equipment and methodology capable of reliably quantifying the canine PLR.

2. Characterize the normal canine PLR under varying stimulus conditions including a range of intensities, colors, and flash durations.

3. Describe PLR deficits associated with CLN2 in the dog and evaluate the PLR as a potential biomarker of CLN2.

4. Evaluate the efficacy of TPP1 enzyme replacement therapy in CLN2 Dachshunds using PLR recordings. 


\section{CHAPTER 2}

\section{INSTRUMENTATION AND RECORDING METHODS DEVELOPMENT}

\subsection{Introduction}

Canine homologues of human hereditary retinal and neurological diseases have potential to serve as important models for developing better understanding of the mechanisms underlying disease and for testing treatment approaches. Mutations for many of these diseases have been identified since the canine genome became available (Veske et al. 1999; M. Katz et al. 2008; Acland et al. 1999). While electroretinography (ERG) is commonly used to assess retinal function in both dogs and people, the ERG only evaluates a small portion of the pathways involved in retinal mediated responses to light stimuli. Therefore, the

PLR and ERG complement one another for objective quantitative assessment of the retina and the integrity of neural pathways originating in the retina.

To make optimal use of dogs as models for diseases affecting function of the retina and associated central nervous system pathways, objective noninvasive measures to assess the functional integrity of these pathways, would be of great benefit. Although the PLR has been widely used in people, pupillography has not been extensively studied in dogs or other large animals due to several obstacles. Physical restraint of the animal during recording causes stress or anxiety, which can influence the PLR (Lowenstein and Lowenfeld 1950). In addition, with a conscious, unsedated animal it is impossible 
to keep the globe of the eye stationary, as is required for pupillography. Unlike people, animals will not voluntarily maintain visual fixation on a target without extensive training.

To overcome these barriers and facilitate the use of pupillography in evaluation of potential canine models of visual system and other neurological disorders, studies were undertaken to develop a reliable protocol for quantitative assessment of the PLR in dogs under varying stimulus conditions.

\subsection{Methods}

\subsubsection{Animals}

Studies employed normal purpose-bred long-haired miniature Dachshunds $(n=15)$ housed in a research facility at the University of Missouri. Prior to inclusion in the study, all dogs received a complete ophthalmic examination including vision assessment, slit lamp biomicroscopy, indirect ophthalmoscopy and retinal photography. Dogs with evidence of vision compromise or ophthalmic conditions deemed threatening to vision were excluded. Dogs were entrained to a 12:12 daily light cycle and were socialized daily in addition to receiving routine husbandry care. All studies were performed in compliance with the ARVO Statement for the Use of Animals in Ophthalmic and Vision Research and were approved by the University of Missouri Animal Care and Use Committee. 


\subsubsection{Experimental Design}

Dogs underwent PLR recordings using either injectable chemical restraint

alone (sedation, neuroleptanalgesia, or dissociative anesthesia) $(n=8)$, or using a combination of injectable sedation and inhalant anesthesia $(n=7)$. For the group that received injectable chemical restraint alone, each method described below was tested on at least two separate occasions in different dogs. No single dog was subjected to recordings more often than once per week. For dogs that received the combination of injectable chemical restraint and inhalant anesthesia, a standard PLR recording session was repeated in each dog every 2 months from 4 to 12 months of age, for a total of 5 recordings per dog.

\subsubsection{Chemical Restraint for PLR Recording}

Five methods of chemical restraint were assessed during quantitative evaluation of the PLR. Four methods involved injectable chemical restraint only, and the fifth method utilized injectable sedation with inhalant anesthesia. For all methods, 30 minutes of dim light adaptation ( 0.9 lux broad spectrum, diffuse white light) preceded drug administration. The methods employing only injectable chemical restraint are widely used in veterinary medicine and are as follows: sedation with low-dose dexmedetomidine (5 $\mu \mathrm{g} / \mathrm{kg}$ IM; Dexdomitor, Orion Corp., Espoo, Finland), sedation with high-dose dexmedetomidine (35 $\mu \mathrm{g} / \mathrm{kg} \mathrm{IM})$, dissociative anesthesia with dexmedetomidine $(18 \mu \mathrm{g} / \mathrm{kg} \mathrm{IM})$ and ketamine (3.5 mg/kg IM; KetaVed, Vedco Inc., Saint Joseph, MO) or 
neuroleptanalgesia with dexmedetomidine $(5 \mu \mathrm{g} / \mathrm{kg} \mathrm{IM})$ and butorphanol $(0.17$ mg/kg IM; Torbugesic, Fort Dodge Animal Health, Overland Park, KS).

For the combined injectable chemical restraint and inhalant anesthesia, dexmedetomidine $(20-25 \mu \mathrm{g} / \mathrm{kg}$ IM) was given 30 minutes prior to induction of anesthesia with propofol (IV to effect, $1.49 \pm 0.59 \mathrm{mg} / \mathrm{kg}$ (mean \pm SD); PropoFlo 28, Abbott Laboratories, Abbott Park, IL). Dogs were intubated with a cuffed endotracheal tube and anesthesia maintained with isoflurane (1.5\% vaporizer setting; Terrell, Piramal Healthcare, Boise, ID) in oxygen. Vaporizer calibration was verified annually by an authorized anesthetic service technician.

Heart and respiratory rates were monitored throughout all procedures, and body temperature was maintained between $98^{\circ} \mathrm{F}$ and $101.5^{\circ} \mathrm{F}$ using a heated circulating water blanket. In addition during general anesthesia, dogs received intravenous fluids ( $5 \mathrm{ml} / \mathrm{kg} / \mathrm{hour}$; veterinary lactated ringer's injection USP), and a portable multiparameter veterinary monitor (Cardell 9500 HD, Midmark Corp., Versailles, $\mathrm{OH}$ ) was used to assess blood pressure, oxygen saturation, and endtidal $\mathrm{CO}_{2}$ levels throughout the procedure.

The methods of chemical restraint were evaluated with regard to whether the level of restraint was adequate to perform the required procedures without causing stress to the dog. Signs of stress in the dog included a sudden increase in respiration or heart rate and physical resistance to the placement of a speculum or stay suture. Stability of the baseline pupil size was also evaluated. 


\subsubsection{PLR Recording Apparatus}

Recordings were performed with a custom apparatus (Figure 2-1) capable of timed delivery of a visible light stimulus from a mounted high-power light-emitting diode (LED) (MCWHL2/white, M625L2/red, M470L2/blue; Thorlabs Inc., Newton, $\mathrm{NJ}$ ) and concurrent recording of pupil images at 30 frames per second using an infrared-sensitive camera (PC164CEX-2; Supercircuits Inc., Austin, TX) and continuous infrared illumination ( $880 \mathrm{~nm}$ LED) for visualization of the eye. Stimulus delivery and image capture were synchronized via an external data acquisition module and image acquisition PCI card and controlled by a custom software program written in LabVIEW (National Instruments Corp., Austin, TX). The system design is based on that of a custom pupillometer intended for human application (see Appendix A for complete parts list) (Fan et al. 2009b).

\subsubsection{PLR Recording Methods}

All recordings were done during the light period of the daily 12:12 light-dark cycle. Dogs were kept in dim white light (0.9 lux) for at least 1 hour, including preparation time, and in complete darkness for 10 minutes prior to recording. After 30 minutes of dim light adaptation, dogs were subjected to either sedation alone or underwent general anesthesia as described above. For all PLR recording sessions, the dog was positioned in sternal recumbency, with the head placed on a deflatable cushion. The cornea of the right eye was anesthetized ( $0.5 \%$ proparacaine hydrochloride, Alcon Laboratories, Fort Worth, TX) and an 
eyelid speculum was inserted to ensure that the nictitating membrane and eyelids did not interfere with light exposure or visualization of the pupil. A small stay suture of 5-0 silk was placed in the bulbar conjunctiva on the central axis approximately $5 \mathrm{~mm}$ superior to the limbus to facilitate globe manipulation to maintain centration of the pupil on the optical axis of the recording apparatus. Suture ends were left long to be threaded between the outer surface of the eyelid and the lid speculum and clamped with a hemostat, which was draped over the top of the dog's head and taped to the deflatable cushion in order to maintain a constant pupil position throughout recording (Figure 2-1). The eye was regularly lubricated with saline eye wash solution throughout the procedure.
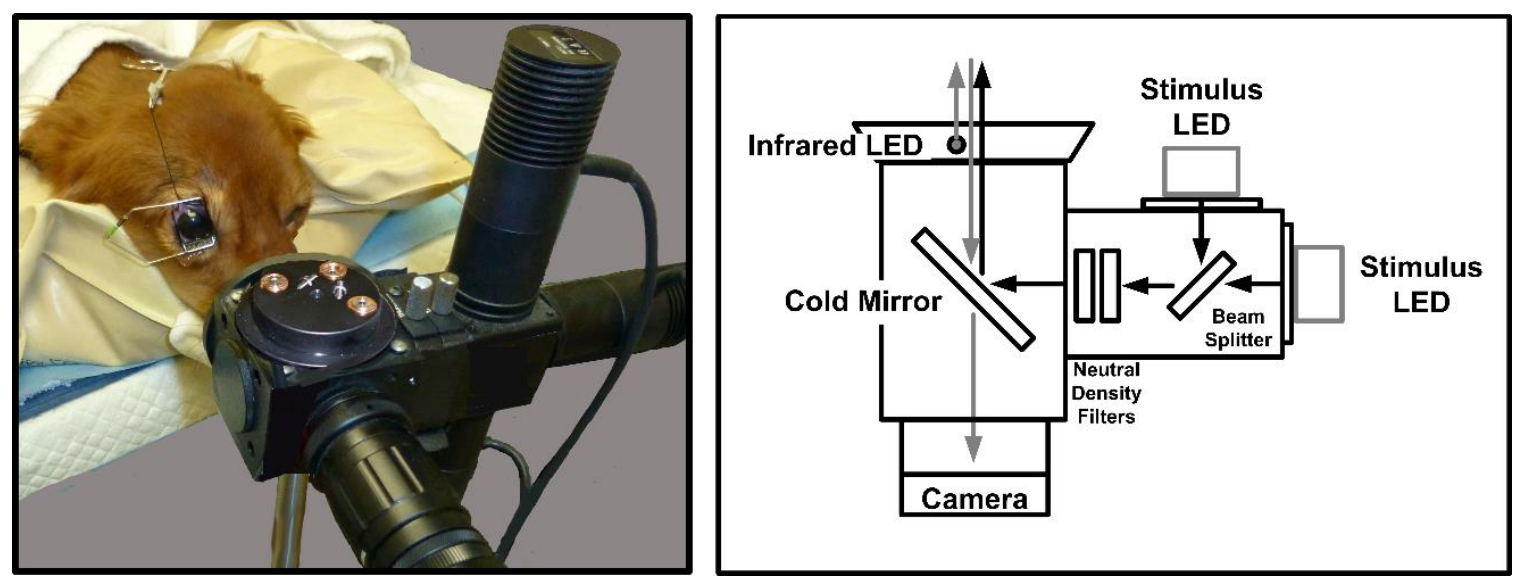

Figure 2-1: The camera and stimulus delivery portion of the PLR recording apparatus (left) and its schematic diagram (right). See Appendix A for full parts list. Photograph was taken in normal room light after the procedure.

The camera and LED stimulus delivery portion of the recording apparatus was mounted on a tripod and positioned $10 \mathrm{~cm}$ from the cornea (Figure 2-1) with the pupil centered in the image frame. A piece of black fabric was draped over 
the dog's head and the camera throughout the procedure to ensure complete darkness, and the fabric was positioned directly over the untested eye to prevent any indirect pupil response in the eye of interest.

\subsubsection{PLR Stimulus Protocols}

The direct PLR of the right eye was evaluated using a standardized protocol (Table 2-1) of 100 millisecond flashes of white light at each of 10 intensities between 8 and 15 log photons $/ \mathrm{cm}^{2} / \mathrm{s}$ (0.1 to 1400 lux). In order to investigate the response of intrinsically photosensitive retinal ganglion cells (ipRGCs), the duration and color of the stimuli were altered for a second set of stimuli (Park et al. 2011; Gamlin et al. 2007). PLRs were recorded in response to 1 second duration blue light stimuli (470 nm peak; $14.5 \mathrm{~nm}$ half-width) and 1 second duration red light stimuli ( $627 \mathrm{~nm}$ peak; $8.5 \mathrm{~nm}$ half-width). Both red and blue stimuli were tested at two intensities, 13 and $14.6 \log$ photons $/ \mathrm{cm}^{2} / \mathrm{s}$.

Irradiance $\left(\mu \mathrm{W} / \mathrm{cm}^{2} / \mathrm{nm}\right)$ and illuminance (lux) measurements were performed using a NIST-traceable calibrated spectroradiometer (ILT950, International Light Technologies, Peabody, MA) and irradiance values were subsequently converted to photon flux for the visible range. Pupil images were recorded for 5 seconds before each flash and for 15 to 85 seconds after the flash depending on intensity of the light. Time between flashes ranged from 1 minute with the dimmest flash to 4 minutes with the brightest flash to allow the pupil to return to baseline size. After dark-adaptation and positioning of the eye, the recording protocol required approximately 1 hour. 


\begin{tabular}{|c|c|c|c|c|}
\hline \multicolumn{2}{|c|}{ Stimulus Intensity } & \multirow{2}{*}{$\begin{array}{l}\text { Time between } \\
\text { flashes }\end{array}$} & \multirow{2}{*}{$\begin{array}{l}\text { Recording } \\
\text { Time }\end{array}$} & \multirow{2}{*}{ \# of trials } \\
\hline $\begin{array}{c}\text { log photons/ } \\
\mathrm{cm}^{2} / \mathrm{s}\end{array}$ & lux & & & \\
\hline 8 & 0.10 & 1 minute & 30 seconds & 3 \\
\hline 8.5 & 0.12 & 1 minute & 30 seconds & 3 \\
\hline 8.75 & 0.15 & 1 minute & 30 seconds & 3 \\
\hline 9 & 0.18 & 1 minute & 30 seconds & 3 \\
\hline 10 & 0.21 & 1.5 minutes & 45 seconds & 3 \\
\hline 11 & 0.30 & 2 minutes & 45 seconds & 3 \\
\hline 12 & 1.2 & 3 minutes & 60 seconds & 2 \\
\hline 13 & 10.8 & 3 minutes & 60 seconds & 2 \\
\hline 14 & 118 & 4 minutes & 90 seconds & 2 \\
\hline 15 & 1400 & 4 minutes & 90 seconds & 2 \\
\hline
\end{tabular}

Table 2-1: PLR stimulus protocol. All stimuli were presented as a 100 millisecond flash of white light beginning with the dimmest flash and gradually increasing as outlined in the table.

\subsubsection{Baseline Pupil Measurements}

Four conscious, unrestrained dogs were dim light and dark adapted as described above. Baseline pupil images were then captured using the infrared camera. Holding the camera by hand, a short series of pupil images could be captured without restraining the dogs.

\subsubsection{PLR Image Analysis}

Pupil images were analyzed using the batch processing feature in Photoshop (CS6 extended; Adobe Systems Inc; San Jose, CA). An "action" was created to calculate pupil area with a series of user-recorded steps (see Appendix B for detailed description). First, the pupil was selected by defining a 
pixel "color range" (Figure 2-2A). The selection was then modified by the smooth command to eliminate extraneous pixels and then expanded and contracted by equal amounts to eliminate the hole in the selection caused by the reflection of light from the infrared LED off the surface of the cornea (Figure 2-2B). A list of image frame number and corresponding pupil area was exported to a spreadsheet and used to calculate desired parameters. Area measurements were converted from pixels to $\mathrm{mm}^{2}$ based on the known size of the lid speculum present in each pupil image (Figure 2-2).
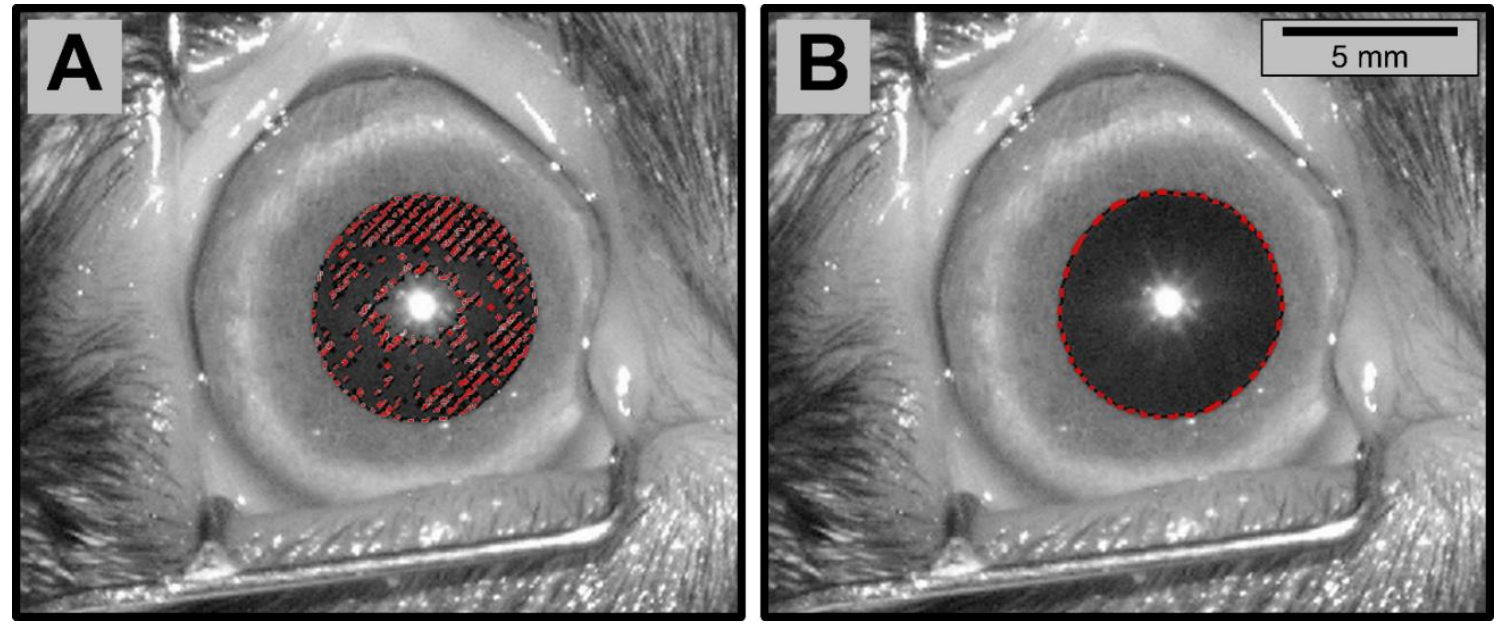

Figure 2-2: Photoshop analysis showing selection of the pupil (red hash marks) based on pixel color (A). After expanding and contracting the selection by equal amounts, the hole in the selection caused by the infrared reflection is removed leaving an accurate outline of the pupil (B).

\subsubsection{Chemical Restraint for Electroretinography}

Dogs were deeply sedated with intramuscular administration of dexmedetomidine (30 - $40 \mu \mathrm{g} / \mathrm{kg}$ ). Ketamine (up to $5 \mathrm{mg} / \mathrm{kg}$ ) was used in combination with the dexmedetomidine for dogs 6 months of age and younger to 
achieve adequate sedation. It was omitted in older dogs to reduce the risk of seizure associated with LINCL, and it was no longer necessary for adequate sedation with this age group. Heart and respiratory rates were closely monitored throughout the procedure, and the dog was temperature controlled with a heated circulating water blanket.

\subsubsection{Electroretinography}

Beginning at 3 months of age, bilateral ERG evaluations were performed monthly using the Dog Diagnostic Protocol as described below (Katz et al. 2008). For ages at which dogs underwent PLR recording, ERG evaluation was performed within one week after the PLR recording session. Prior to sedation, pupils were dilated with topical ophthalmic administration of $1 \%$ tropicamide, and dogs were prepared for recording in normal room light. The dog's head was positioned on a deflatable cushion to ensure complete stability. The cornea was anesthetized with $1 \%$ proparacaine (Alcaine; Alcon), and a lid speculum was inserted to ensure that the nictitating membrane and the upper and lower eyelids did not interfere with light exposure of the maximally dilated pupils. In addition, a small stay suture was placed in the bulbar conjunctiva on the central axis approximately $5 \mathrm{~mm}$ superior to the limbus to facilitate globe manipulation to maintain centration of the pupil. Platinum subdermal needle electrodes (Model E2; Grass Instrument Division, Astro-Med, Inc., West Warwick, RI) were used for the ground electrode, positioned on the occipital crest, and for the reference electrodes, positioned 3 to $4 \mathrm{~cm}$ from the lateral canthus, close to the base of 
each ear. An active contact lens electrode (ERG-Jet; Universo Plastique, LKC Technologies Inc., Gaithersburg, MD) was placed on each cornea after instillation of one drop of $2 \%$ methylcellulose. The electrodes were connected to a preamplifier, and the signals were amplified with a band-pass filter between 0.3 and $300 \mathrm{~Hz}$.

\subsubsection{ERG Dog Diagnostic Protocol}

ERGs were bilaterally elicited and simultaneously recorded with a portable unit (HMsERG model 2000; RetVet Corp., Columbia, MO). The right and left mini-ganzfeld domes were positioned approximately $2 \mathrm{~cm}$ from the corresponding eye. The Dog Diagnostic Protocol is pre-programmed on the HmsERG unit and consists of scotopic and photopic ERGs, as recommended by the European College of Veterinary Ophthalmology, primarily for evaluation of rod and cone function (Narfström et al. 2002). The details of the stimulus protocol are outlined in Table 2-2. During 20 minutes of dark adaptation, scotopic low-intensity rod responses were elicited every 4 minutes at a stimulus intensity of $0.01 \mathrm{~cd} . \mathrm{s} / \mathrm{m}^{2}$. Thereafter, scotopic responses were elicited using 3 $\mathrm{cd} . \mathrm{s} / \mathrm{m}^{2}$ and $10 \mathrm{~cd}-\mathrm{s} / \mathrm{m}^{2}$ flashes to evaluate mixed rod and cone function. After 10 minutes of light adaptation with a background luminance of $30 \mathrm{~cd} . \mathrm{s} / \mathrm{m}^{2}$, photopic single-flash responses were recorded using $3 \mathrm{~cd} . \mathrm{s} / \mathrm{m}^{2}$ of flash stimulus, followed by evaluation of $30-\mathrm{Hz}$ photopic flicker at the same light intensity. The latter two recordings were performed to evaluate cone and inner retina function, respectively. 


\begin{tabular}{|c|c|c|c|c|c|}
\hline \multirow[b]{2}{*}{ Session } & \multicolumn{2}{|c|}{ Stimulus Intensity } & \multirow{2}{*}{$\begin{array}{l}\text { Session } \\
\text { Duration }\end{array}$} & \multirow{2}{*}{$\begin{array}{l}\text { Time } \\
\text { Between } \\
\text { Flashes }\end{array}$} & \multirow{2}{*}{$\begin{array}{c}\text { \# of } \\
\text { averaged } \\
\text { flashes }\end{array}$} \\
\hline & $\begin{array}{c}\log \\
\text { photons/ } \\
\mathrm{cm}^{2} / \mathbf{s}\end{array}$ & cd.s $/ \mathrm{m}^{2}$ & & & \\
\hline Dark adaptation 1 & & & $4 \mathrm{~min}$. & & \\
\hline Rods recording 1 & 10.2 & 0.01 & & $2 \mathrm{sec}$. & 10 \\
\hline Dark adaptation 2 & & & $4 \mathrm{~min}$. & & \\
\hline Rods recording 2 & 10.2 & 0.01 & & $2 \mathrm{sec}$. & 10 \\
\hline Dark adaptation 3 & & & $4 \mathrm{~min}$. & & \\
\hline Rods recording 3 & 10.2 & 0.01 & & $2 \mathrm{sec}$. & 10 \\
\hline Dark adaptation 4 & & & $4 \mathrm{~min}$. & & \\
\hline Rods recording 4 & 10.2 & 0.01 & & $2 \mathrm{sec}$. & 10 \\
\hline Dark adaptation 5 & & & $4 \mathrm{~min}$. & & \\
\hline Rods recording 5 & 10.2 & 0.01 & & $2 \mathrm{sec}$. & 10 \\
\hline Delay & & & $2 \mathrm{sec}$. & & \\
\hline $\begin{array}{c}\text { Rods \& Cones - } \\
\text { Standard Intensity }\end{array}$ & 12.65 & 3 & & $10 \mathrm{sec}$. & 4 \\
\hline Delay & & & $30 \mathrm{sec}$ & & \\
\hline $\begin{array}{l}\text { Rods \& Cones - } \\
\text { Hi Intensity }\end{array}$ & 13.2 & 10 & & $20 \mathrm{sec}$. & 4 \\
\hline Light Adaptation & 13.65 & 30 & $10 \min$ & & \\
\hline Cones & 12.65 & 3 & & $0.5 \mathrm{sec}$ & 32 \\
\hline Delay & & & $2 \mathrm{sec}$ & & \\
\hline Flicker - $30 \mathrm{~Hz}$ & 12.65 & 3 & $4.1 \mathrm{sec}$ & 0.032 & 128 \\
\hline
\end{tabular}

Table 2-2: ERG Dog Diagnostic Protocol. Photon flux values (log photons $/ \mathrm{cm}^{2} / \mathrm{s}$ ) were calculated from irradiance levels measured with a NIST-traceable spectroradiometer. 


\subsubsection{ERG Scotopic Intensity Series}

For comparison of the ERG and PLR thresholds, a scotopic intensity series was performed in the right eye of 5 dogs. Dogs were dim light adapted for 1 hour and dark adapted for 10 minutes prior to ERG recording; this is the same protocol used in PLR recording. During dim light adaptation, dogs were prepared for recording as described in the previous section. The scotopic 0 , scotopic 1 , and scotopic 2 intensity series (Table 2-3) are pre-programmed on the HmsERG unit and require the use of neutral density filters (ND 3 for Scotopic 0 and ND 2 for Scotopic 1). The intensity series stimuli ranged from $0.03 \mathrm{mcd} . \mathrm{s} / \mathrm{m}^{2}$ to 30 cd.s $/ \mathrm{m}^{2}\left(7.65\right.$ to 13.65 log photons $\left./ \mathrm{cm}^{2} / \mathrm{s}\right)$ with a 60 second delay between intensities. 


\begin{tabular}{|c|c|c|c|c|c|}
\hline \multirow[b]{2}{*}{ Session } & \multicolumn{2}{|c|}{ Stimulus Intensity } & \multirow{2}{*}{$\begin{array}{c}\text { Time } \\
\text { Between } \\
\text { Flashes }\end{array}$} & \multirow{2}{*}{$\begin{array}{c}\text { \# of } \\
\text { averaged } \\
\text { flashes }\end{array}$} & \multirow{2}{*}{$\begin{array}{l}\text { Delay } \\
\text { after } \\
\text { session }\end{array}$} \\
\hline & $\begin{array}{c}\log \\
\text { photons/ } \\
\text { cm }^{2} / \mathbf{s}\end{array}$ & mcd.s $/ \mathrm{m}^{2}$ & & & \\
\hline \multirow[t]{7}{*}{ Scotopic 0} & 7.65 & 0.03 & $2 \mathrm{sec}$. & 10 & $60 \mathrm{sec}$. \\
\hline & 8.2 & 0.1 & $2 \mathrm{sec}$. & 10 & $60 \mathrm{sec}$. \\
\hline & 8.65 & 0.3 & $2 \mathrm{sec}$. & 10 & $60 \mathrm{sec}$. \\
\hline & 9.2 & 1.0 & $2 \mathrm{sec}$. & 4 & $60 \mathrm{sec}$. \\
\hline & 9.65 & 3 & $2 \mathrm{sec}$. & 4 & $60 \mathrm{sec}$. \\
\hline & 10.2 & 10 & $2 \mathrm{sec}$. & 4 & $60 \mathrm{sec}$. \\
\hline & 10.65 & 30 & $10 \mathrm{sec}$. & 4 & 4 min. \\
\hline \multirow[t]{5}{*}{ Scotopic 1} & 8.2 & 0.1 & $2 \mathrm{sec}$. & 10 & $60 \mathrm{sec}$. \\
\hline & 9.2 & 1.0 & $2 \mathrm{sec}$. & 10 & $60 \mathrm{sec}$. \\
\hline & 10.2 & 10 & $2 \mathrm{sec}$. & 10 & 60 sec. \\
\hline & 10.65 & 30 & $10 \mathrm{sec}$. & 4 & 60 sec. \\
\hline & 11.2 & 100 & $10 \mathrm{sec}$. & 4 & 4 min. \\
\hline \multirow[t]{6}{*}{ Scotopic 2} & 11.2 & 100 & $10 \mathrm{sec}$. & 4 & $60 \mathrm{sec}$. \\
\hline & 11.65 & 300 & $10 \mathrm{sec}$. & 4 & $60 \mathrm{sec}$. \\
\hline & 12.2 & 1000 & $10 \mathrm{sec}$. & 4 & $60 \mathrm{sec}$. \\
\hline & 12.65 & 3000 & $10 \mathrm{sec}$. & 4 & 60 sec. \\
\hline & 13.2 & 10000 & & 1 & $60 \mathrm{sec}$. \\
\hline & 13.65 & 30000 & & 1 & \\
\hline
\end{tabular}

Table 2-3: ERG scotopic intensity series protocol. Photon flux values (log photons $/ \mathrm{cm}^{2} / \mathrm{s}$ ) were calculated from irradiance levels measured with a NIST-traceable spectroradiometer. 


\subsubsection{Visually Evoked Potentials}

Immediately following an ERG session in which the dog had already been light adapted, the room lights were turned on (14.5 log photons $/ \mathrm{cm}^{2} / \mathrm{s}$ or $400 \mathrm{lux}$ ) and the electrodes were repositioned for VEP recording. Subdermal needle electrodes were placed over the occipital crest (active), on the forehead (reference), and $3-4 \mathrm{~cm}$ from the lateral canthus, close to the base of the right ear (ground). VEPs were elicited bilaterally with $12.65 \mathrm{log}$ photon $/ \mathrm{cm}^{2} / \mathrm{s}$ flashes $\left(3 \mathrm{~cd} . \mathrm{s} / \mathrm{m}^{2}\right) ; 30$ flashes were presented at $1 \mathrm{HZ}$ and the results averaged. This protocol was repeated three times for a total of 90 flashes. The averaged responses were evaluated for amplitude and implicit time.

\subsubsection{ERG and VEP Waveform Analysis}

ERG waveforms in all recordings were evaluated, and the amplitudes and implicit times for the a- and b-waves were measured as illustrated in Figure 2-3 (Marmor et al. 2004). VEP waveforms were analyzed for amplitude and implicit time of the peak occurring between 40 and 50 milliseconds after the flash (Figure 2-4). For analysis, data files were transferred to a computer from the memory card of the HmsERG unit and opened with the ERGVIEW software (v. 3220, Occuscience LLC, Kansas City, MO). Amplitude and implicit time measurements were made using this software and then exported as a text file for further calculations. 


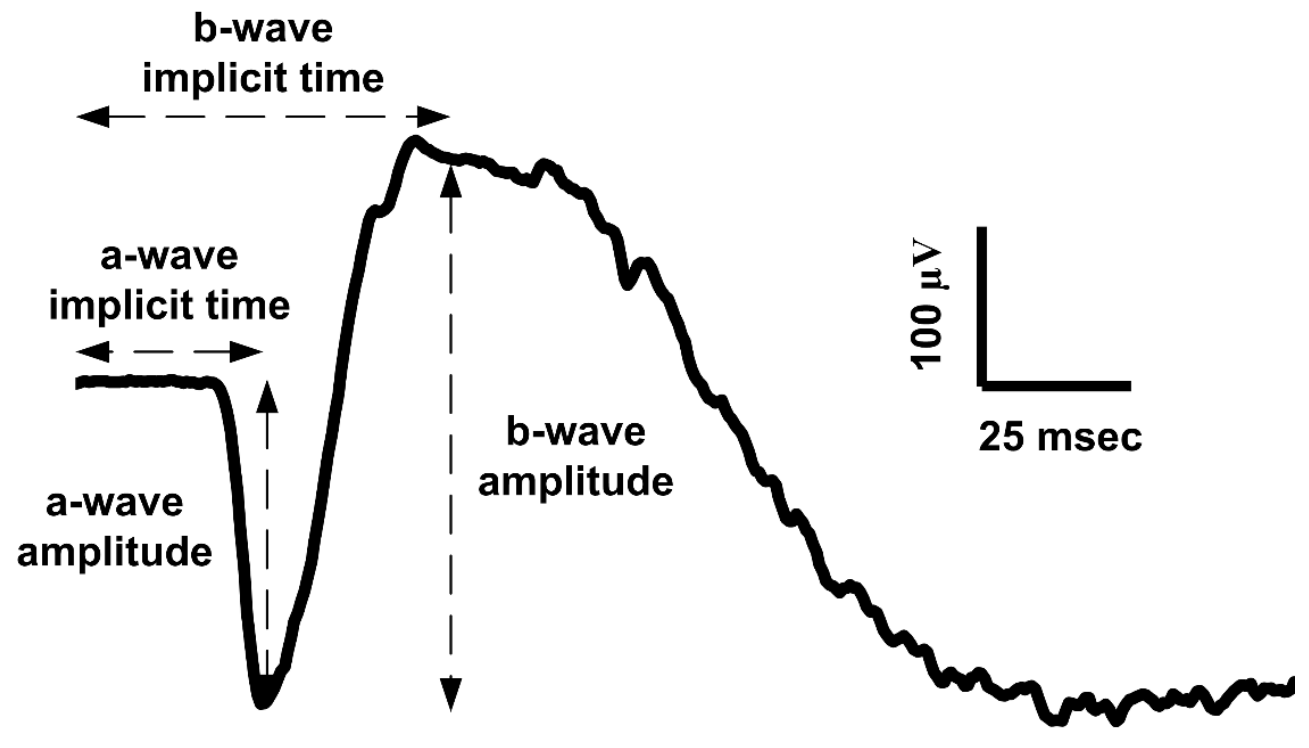

Figure 2-3: ERG waveform analysis. The a-wave amplitude is measured as from the baseline to the trough of the a-wave, and the b-wave amplitude is measured from the trough of the a-wave to the peak of the b-wave. The a-wave and b-wave implicit times are measured as the time from onset of the flash stimulus to the trough of the a-wave or the peak of the b-wave, respectively.

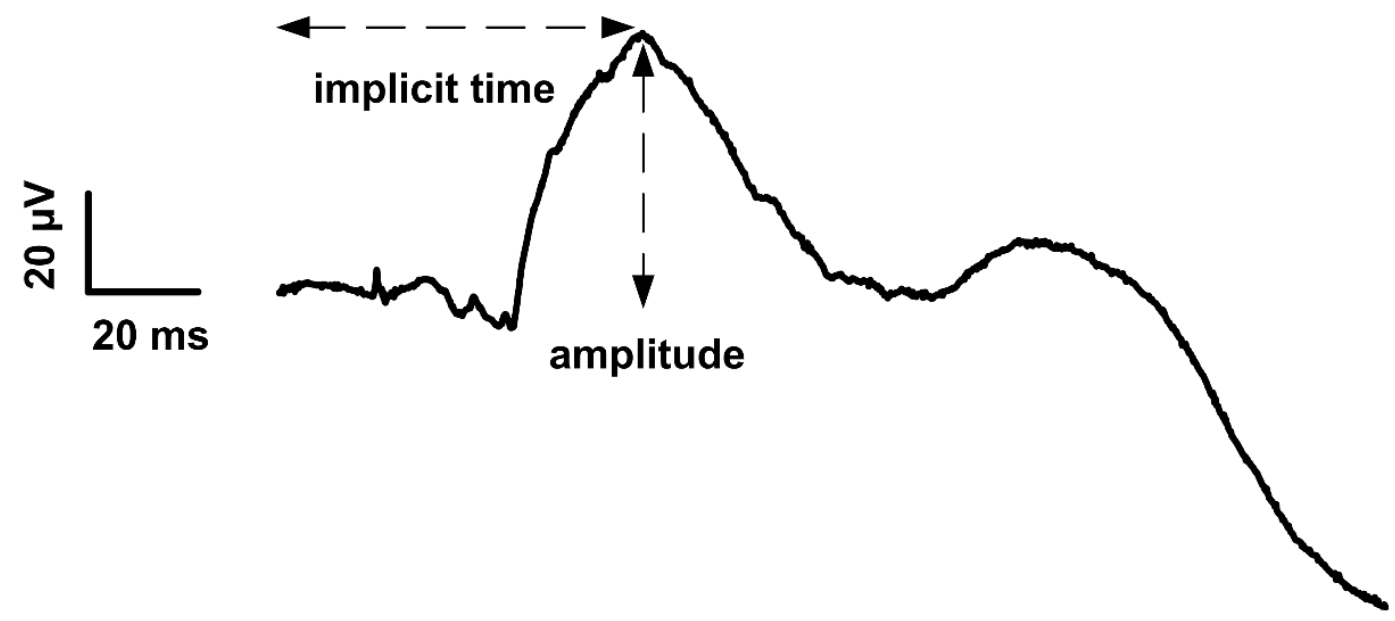

Figure 2-4: VEP waveform analysis. The amplitude was measured for the peak occurring between 40 and 50 milliseconds after the flash. Implicit time was measured as the actual time from the flash to the measured peak. 


\subsection{Results}

\subsubsection{Effects of Sedation and General Anesthesia on the PLR}

With injectable chemical restraint alone, large spontaneous fluctuations in pupil size occurred independent of light stimulation (Figure 2-5). This fluctuation was quantified by calculating the average pupil size from 20 seconds of baseline recording, calculating the deviation of each data point from this value, and computing the average deviation (Table 2-4). Range was calculated as the difference between the maximum and minimum pupil size during the 20 second baseline recording (Table 2-4). Ventral rotation of the eye was also common, making visualization of the entire pupil difficult or impossible. In all cases, eyelid interference with imaging was a problem and necessitated the use of an eyelid speculum. However, low-dose dexmedetomidine did not provide adequate sedation to prevent the dog from resisting speculum placement. In addition, under this light sedation the eye was only stationary for very short periods making it difficult to capture even a 10 second series of images.

The level of anesthesia achieved with combined injectable sedation and inhalant anesthesia was characterized by ventral rotation of the eye globes and a lack of response to external tactile and auditory stimuli. This protocol produced a very consistent baseline pupil size with minimal noise (Figure 2-5D) and allowed for the use of an eyelid speculum and conjunctival stay suture for stable positioning of the eye without signs of stress in the animal. 


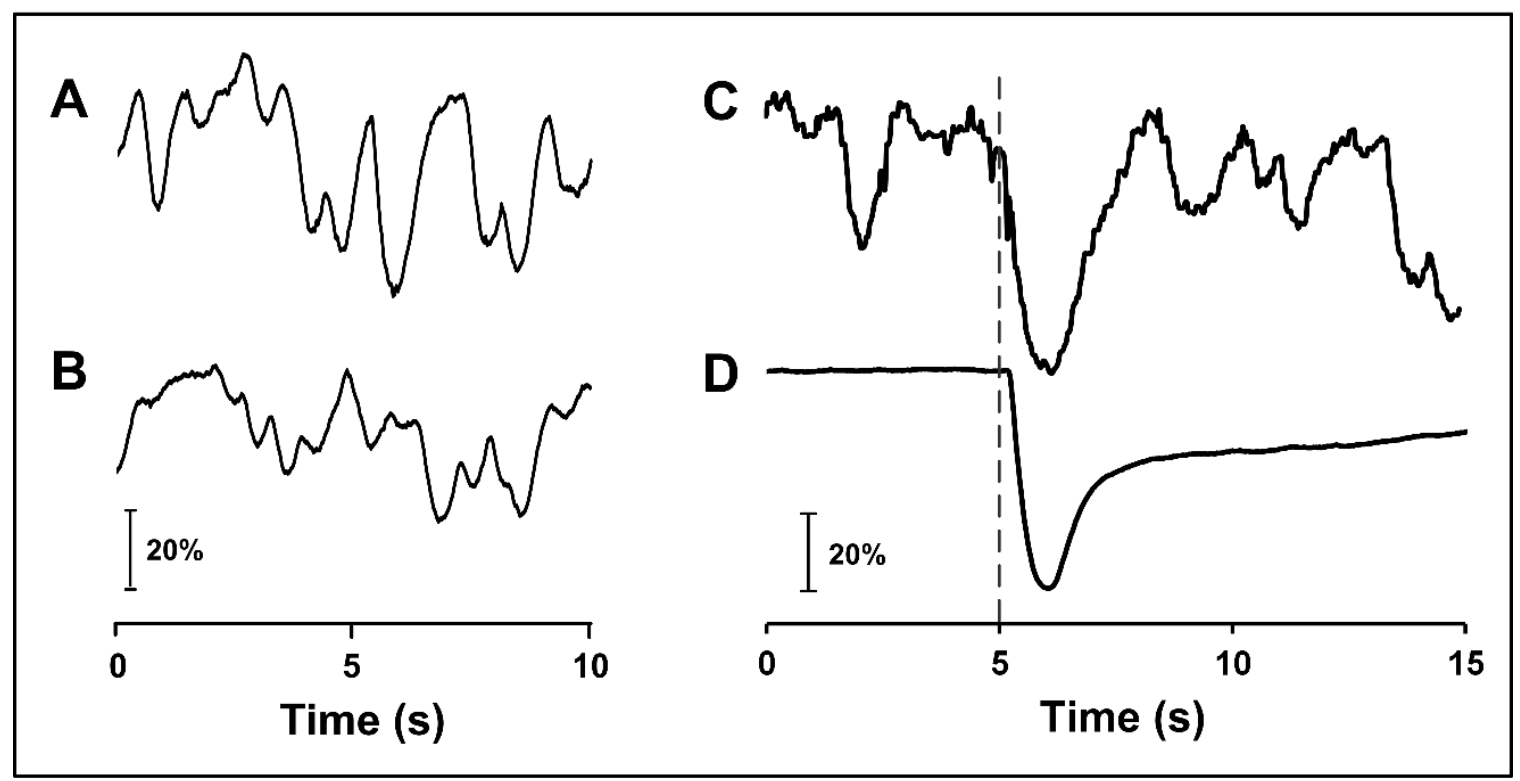

Figure 2-5: Baseline pupil size with different chemical restraint protocols; dexmedetomidine and ketamine $(A)$ and dexmedetomidine and butorphanol $(B)$. PLR traces from a dog under high-dose dexmedetomidine sedation (C) and a dog under general anesthesia (D). Pupil responses are shown as percent of baseline pupil area. No stimulus was used in traces (A) and (B). A 100 millisecond flash stimulus of $11 \mathrm{log}$ photons $/ \mathrm{cm}^{2} / \mathrm{s}$ was given 5 seconds into (C) and (D) recordings (dashed line) resulting in a large constriction. The substantial spontaneous fluctuation in pupil size observed under injectable chemical restraint $(A-C)$ was completely eliminated when the dog was anesthetized (D).

\begin{tabular}{|l|c|c|c|c|}
\hline \multicolumn{1}{|c|}{ Protocol } & $\begin{array}{c}\text { \# of } \\
\text { trials }\end{array}$ & Age (months) & $\begin{array}{c}\text { \% Pupil Fluctuation } \\
\text { (Avg } \pm \text { SD) }\end{array}$ & $\begin{array}{c}\text { Range (\%) } \\
\text { (Avg } \pm \text { SD) }\end{array}$ \\
\hline $\begin{array}{l}\text { dexmedetomidine } \\
\text { (high dose) }\end{array}$ & 5 & $6.8 \pm 1.4$ & $14.4 \pm 1.8$ & $49.0 \pm 9.8$ \\
\hline $\begin{array}{l}\text { dexmedetomidine / } \\
\text { ketamine }\end{array}$ & 4 & $9.2 \pm 0.5$ & $10.6 \pm 4.2$ & $46.1 \pm 0.2$ \\
\hline $\begin{array}{l}\text { dexmedetomidine / } \\
\text { butorphanol }\end{array}$ & 2 & $8.0 \pm 1.5$ & $13.6 \pm 3.7$ & $47.3 \pm 1.5$ \\
\hline isoflurane & 7 & $10 \pm 0.1$ & $0.8 \pm 0.5$ & $2.7 \pm 1.3$ \\
\hline
\end{tabular}

Table 2-4: Baseline pupil fluctuation as a percent of average pupil size for the chemical restraint protocols tested. Range is the difference between the maximum and minimum pupil size over a 20 second baseline recording. Lowdose dexmedetomidine did not allow for reliable recordings making it impossible to quantify these measures. 
The differences between chemical restraint alone and the addition of inhalant anesthesia were further illustrated by a trial in which the dog was sedated prior to general anesthesia and baseline recordings were taken before starting isoflurane administration and at timed intervals after initiating isoflurane administration. The pupil was small and fluctuating in size with sedation alone. Within 10 minutes of beginning isoflurane administration, the pupil almost doubled in size and ceased fluctuating (Figure 2-6).

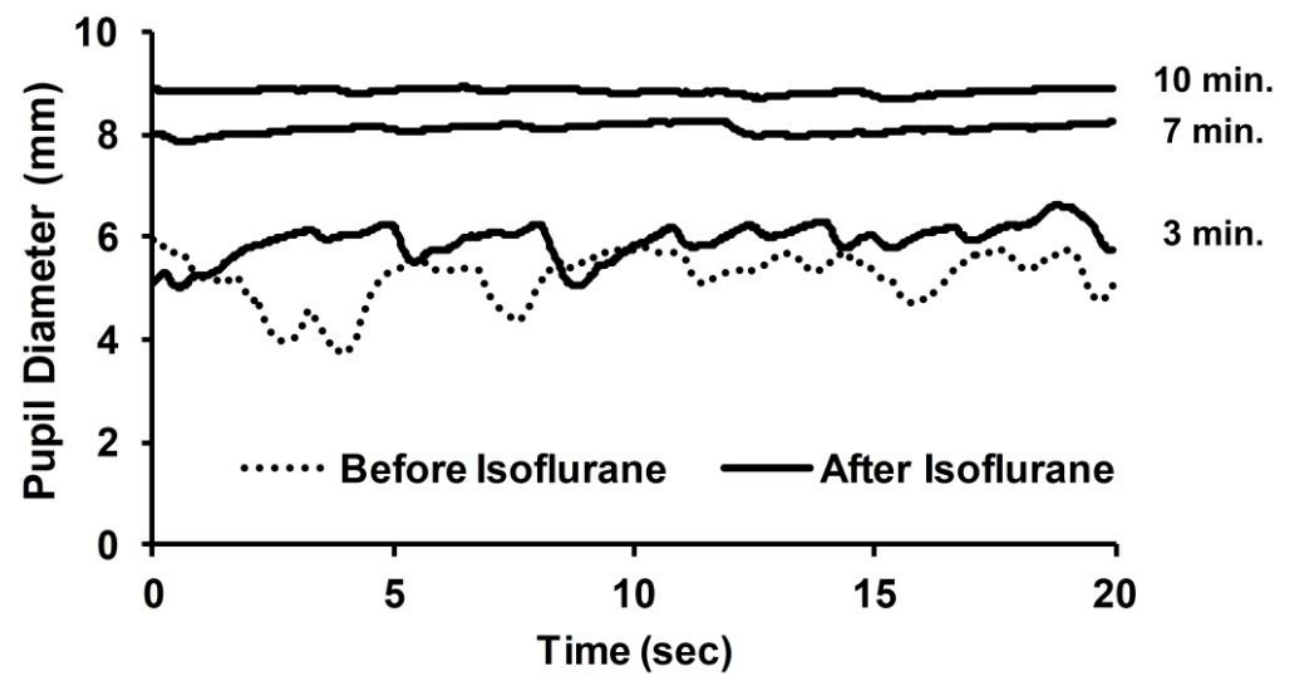

Figure 2-6: Baseline pupil behavior with different chemical restraint protocols. The dashed trace shows the fluctuating pupil size from a dog under dexmedetomidine sedation. The solid traces are from the same $\operatorname{dog} 3,7$, and 10 minutes after isoflurane anesthesia was initiated and show the gradual progression to a stable baseline pupil.

\subsubsection{PLR Recordings}

Average baseline pupil diameter in conscious, unrestrained dogs was 10.06 $\pm 0.54 \mathrm{~mm}$ (mean \pm SEM). Baseline pupil diameter under general anesthesia 
was $8.04 \pm 0.16 \mathrm{~mm}$, which was a significant reduction from that of awake dogs $(p<0.001)$. With a steady baseline pupil size under general anesthesia, it was possible to detect and reliably measure a PLR as small as $5 \%$ constriction. All PLRs recorded under general anesthesia were characterized by four main phases including the steady state baseline pupil prior to the flash stimulus, the rapid pupil constriction in response to the stimulus, the initial redilation phase, and the slower secondary recovery to baseline pupil size (Figure 2-7). As stimulus intensity was increased, the amplitude of pupil constriction also increased (Figure 2-7, Table 2-5). Latency decreased with each increase of stimulus intensity. Average constriction velocity and initial redilation velocity increased with stimulus intensity between 8 and $11 \log$ photons $/ \mathrm{cm}^{2} / \mathrm{s}$, but then declined slightly with each subsequent increase in intensity (Table 2-6). The kinetics of these responses are similar to those of PLRs recorded in people using a similar system.(Fan et al. 2009a) The PIPR was detectable with a stimulus of 11 log photons $/ \mathrm{cm}^{2} / \mathrm{s}$ and increased with each subsequent increase in stimulus intensity (Figure 2-7, Table 2-5).

To assess reproducibility of the PLR constriction amplitudes, the average from all dogs, at each stimulus intensity, was compared between the 5 recording sessions (Table 2-5). For each stimulus intensity between 10 and $15 \log$ photons $/ \mathrm{cm}^{2} / \mathrm{s}$, average constriction amplitude was repeatable across the 5 sessions within a range of $5 \%$. Dim flashes from 8 to 9 log photons $/ \mathrm{cm}^{2} / \mathrm{s}$ produced more variable constriction amplitudes (Table 2-5). 


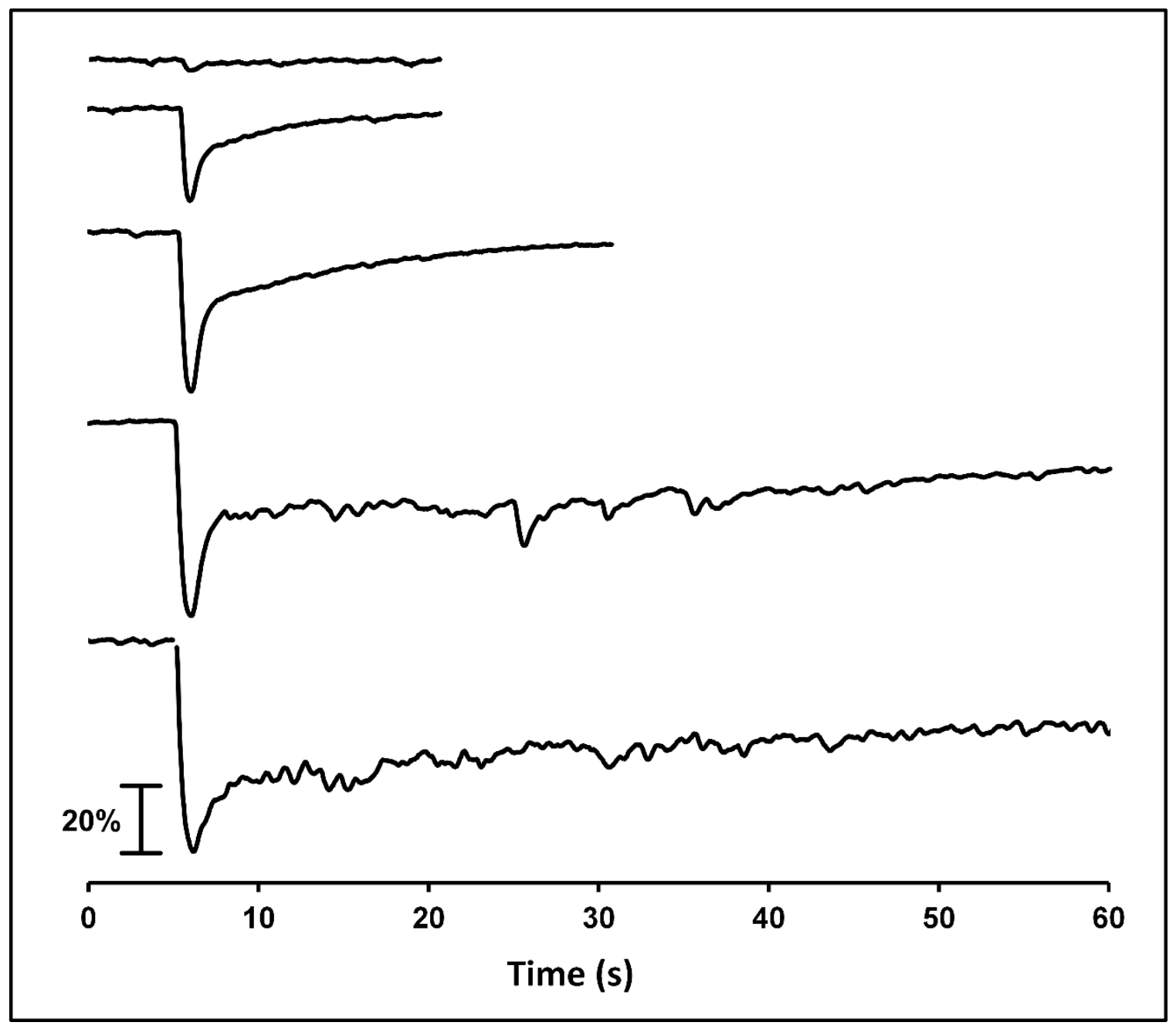

Figure 2-7: PLR traces illustrating the range of pupil constriction observed in anesthetized dogs with 100 millisecond white light stimuli of increasing intensity (from top to bottom: 8, 8.5, 9, 13, and $15 \log$ photons $/ \mathrm{cm}^{2} / \mathrm{s}$ ). Pupil responses are shown as percent of baseline pupil area. The stimulus onset occurred 5 seconds into each recording. 


\begin{tabular}{|c|c|c|c|c|c|c|}
\hline $\begin{array}{c}\text { Stimulus } \\
\text { Intensity } \\
\text { (log } \\
\text { photons/ } \\
\text { cm }^{2} / \mathbf{s} \text { ) }\end{array}$ & $\mathbf{4}$ & $\mathbf{6}$ & $\mathbf{8}$ & $\mathbf{1 0}$ & $\mathbf{1 2}$ & $\begin{array}{c}\text { Aange } \\
\text { (\%) }\end{array}$ \\
\hline $\mathbf{8}$ & $1.05 \pm 0.61$ & $1.24 \pm 0.88$ & $1.81 \pm 1.21$ & $1.36 \pm 0.69$ & $1.86 \pm 1.5$ & 0.81 \\
\hline $\mathbf{8 . 5}$ & $11.94 \pm 4.68$ & $8.46 \pm 2.95$ & $5.92 \pm 4.76$ & $13.46 \pm 5.21$ & $7.6 \pm 2.73$ & 7.53 \\
\hline $\mathbf{8 . 7 5}$ & $18.77 \pm 6.33$ & $17.26 \pm 5.32$ & $8.48 \pm 6.1$ & $24.7 \pm 7.65$ & $15.46 \pm 4.3$ & 16.22 \\
\hline $\mathbf{9}$ & $28.67 \pm 5.28$ & $29.09 \pm 5.19$ & $22.3 \pm 7.5$ & $31.58 \pm 6.37$ & $26.64 \pm 6.37$ & 9.28 \\
\hline $\mathbf{1 0}$ & $40.68 \pm 3.05$ & $42.2 \pm 4.97$ & $38.81 \pm 6.35$ & $43.06 \pm 3.73$ & $39.91 \pm 3.23$ & 4.25 \\
\hline $\mathbf{1 1}$ & $50.51 \pm 2.52$ & $50.47 \pm 3.76$ & $49.69 \pm 4.78$ & $51.15 \pm 2.88$ & $49.52 \pm 2.26$ & 1.63 \\
\hline $\mathbf{1 2}$ & $57.3 \pm 2.56$ & $55.73 \pm 3.47$ & $57.28 \pm 4.3$ & $57.85 \pm 2.35$ & $55.81 \pm 1.81$ & 2.11 \\
\hline $\mathbf{1 3}$ & $58.81 \pm 3.34$ & $58.49 \pm 3.18$ & $63.01 \pm 4.55$ & $62.39 \pm 3.47$ & $59.87 \pm 2.12$ & 4.52 \\
\hline $\mathbf{1 4}$ & $64.41 \pm 5.23$ & $61.71 \pm 3.6$ & $61.83 \pm 5.13$ & $63.28 \pm 2.71$ & $63.2 \pm 3.32$ & 2.70 \\
\hline $\mathbf{1 5}$ & $65.55 \pm 3.96$ & $68.26 \pm 3.78$ & $68.67 \pm 4.47$ & $68.82 \pm 3.4$ & $63.9 \pm 3.3$ & 4.92 \\
\hline
\end{tabular}

Table 2-5: PLR constriction amplitude shown as percent of baseline pupil area (average \pm SEM). Range is the difference between the maximum and minimum average constriction amplitude for the five ages tested.

\begin{tabular}{|c|c|c|c|c|}
\hline \multirow{2}{*}{$\begin{array}{c}\text { Stimulus } \\
\text { Intensity }(\mathbf{l o g} \\
\text { photons/cm } / \mathbf{s})\end{array}$} & Latency (ms) & $\begin{array}{c}\text { Constriction } \\
\text { Speed } \mathbf{( m m / s )}\end{array}$ & $\begin{array}{c}\text { Redilation } \\
\text { Speed (mm/s) }\end{array}$ & PIPR (\%) \\
\cline { 2 - 5 } & $380.56 \pm 26.66$ & $0.5 \pm 0.08$ & $0.16 \pm 0.02$ & -- \\
\hline $\mathbf{8}$ & $328.47 \pm 11.79$ & $1.06 \pm 0.14$ & $0.38 \pm 0.04$ & -- \\
\hline $\mathbf{8 . 5}$ & $283.33 \pm 8.95$ & $1.47 \pm 0.16$ & $0.49 \pm 0.05$ & -- \\
\hline $\mathbf{8 . 7 5}$ & $265.28 \pm 6.96$ & $1.93 \pm 0.15$ & $0.66 \pm 0.06$ & -- \\
\hline $\mathbf{9}$ & $228.45 \pm 3.74$ & $2.54 \pm 0.08$ & $0.91 \pm 0.06$ & $4.72 \pm 1.57$ \\
\hline $\mathbf{1 0}$ & $209.43 \pm 2.98$ & $2.82 \pm 0.06$ & $1.06 \pm 0.06$ & $7.29 \pm 0.79$ \\
\hline $\mathbf{1 1}$ & $198.48 \pm 3.86$ & $2.69 \pm 0.07$ & $0.93 \pm 0.06$ & $17.38 \pm 2.12$ \\
\hline $\mathbf{1 2}$ & $191.4 \pm 3.78$ & $2.53 \pm 0.1$ & $0.85 \pm 0.06$ & $26.77 \pm 2.99$ \\
\hline $\mathbf{1 3}$ & $188.69 \pm 5.08$ & $2.54 \pm 0.11$ & $0.8 \pm 0.07$ & $30.71 \pm 3.48$ \\
\hline $\mathbf{1 4}$ & $183.33 \pm 3.16$ & $2.28 \pm 0.09$ & $0.59 \pm 0.05$ & $44.4 \pm 3.77$ \\
\hline $\mathbf{1 5}$ &
\end{tabular}

Table 2-6: PLR parameters as a function of stimulus intensity in anesthetized dogs. Values from all ages were averaged. 
Red and blue stimuli resulted in robust PLRs with both intensities tested (Figure 2-8). With stimuli of 13 log photons $/ \mathrm{cm}^{2} / \mathrm{s}$, the PLRs to red and blue light were very similar to one another for both the constriction and redilation phases. However, with stimuli of $14.6 \log$ photons $/ \mathrm{cm}^{2} / \mathrm{s}$, red and blue light resulted in very different redilation phases. While the pupil redilated quickly after offset of the red stimulus, pupil constriction persisted after offset of the blue stimulus (Figure 2-8). This sustained constriction was present for at least 60 seconds after the stimulus.

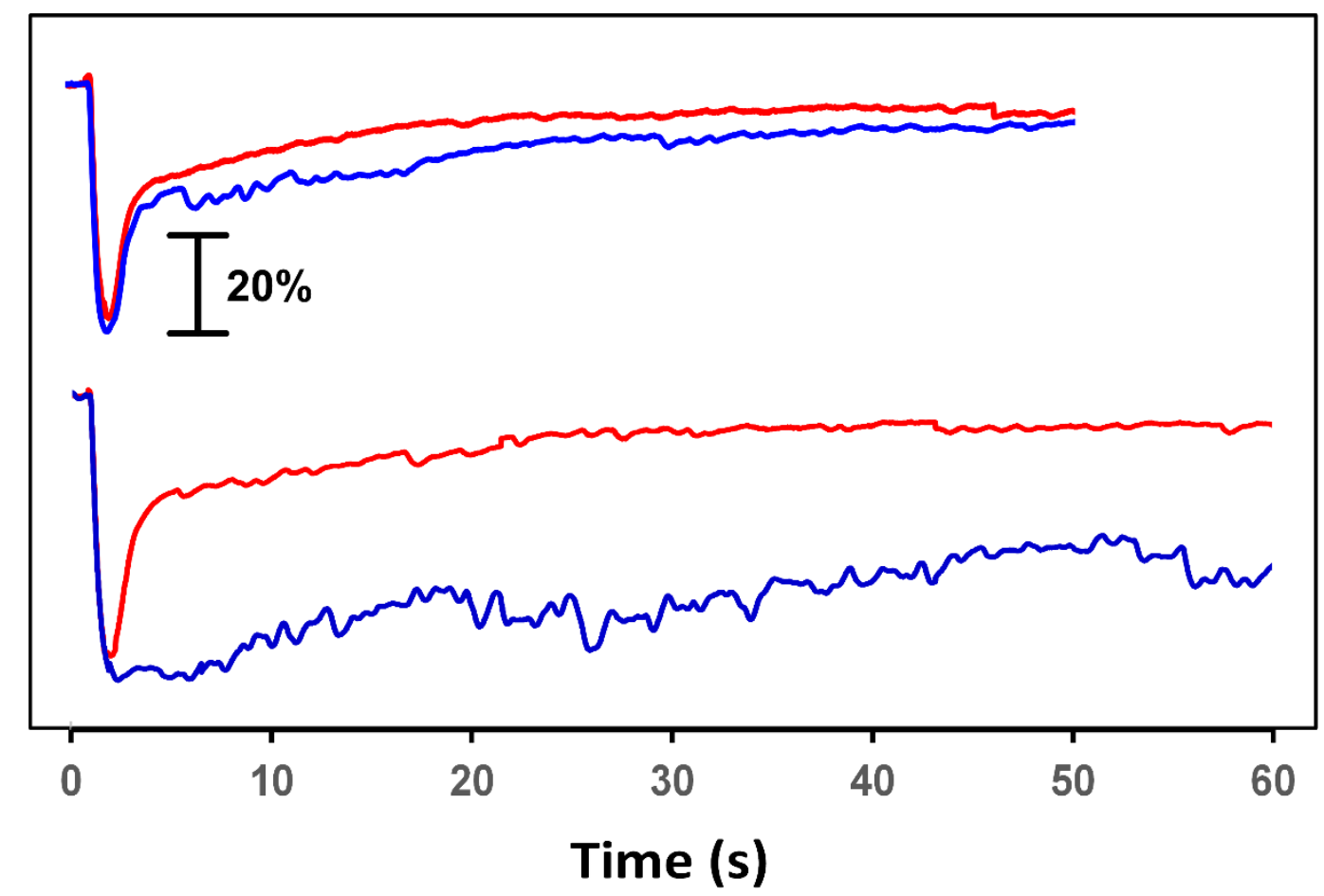

Figure 2-8: Blue and red traces show PLRs to 1 second stimuli of the corresponding color (top, 13 and $13.5 \mathrm{log}$ photons $/ \mathrm{cm}^{2} / \mathrm{s}$; bottom, 14.5 and $15 \mathrm{log}$ photons $/ \mathrm{cm}^{2} / \mathrm{s}$ ). The onset of each stimulus occurred 1 second into each recording. With stimuli of 13 and $13.5 \mathrm{log}$ photons $/ \mathrm{cm}^{2} / \mathrm{s}$, the PLRs to red and blue light are very similar. The brighter blue stimulus (14.5 log photons $\left./ \mathrm{cm}^{2} / \mathrm{s}\right)$ results in sustained pupil constriction, while the pupil quickly redilates after a red stimulus of comparable intensity. Recordings were performed in anesthetized dogs. 


\subsubsection{ERG \& PLR Thresholds}

The ERG assessments demonstrated normal retinal function in all dogs throughout the 8 month period in which PLR recordings were obtained. The threshold stimulus intensity for the PLR was $8.59 \pm 0.30 \mathrm{log}$ photons $/ \mathrm{cm}^{2} / \mathrm{s}$ (average $\pm \mathrm{SD}$ ), while the threshold for the ERG was $9.56 \pm 0.20 \log$ photons $/ \mathrm{cm}^{2} / \mathrm{s}$ (Table $2-7$, Figure 2-9). The stimulus intensity required to elicit a threshold ERG response was significantly higher, by approximately 10 times compared with the stimulus required to produce a reliably detectable pupil constriction $(p<0.00005)$. The minimum intensity required to elicit a recordable awave was $11.2 \log$ photons $/ \mathrm{cm}^{2} / \mathrm{s}$ (Table 2-7).

\begin{tabular}{|c|c|c|c|}
\hline \multicolumn{2}{|c|}{ Stimulus Intensity } & \multicolumn{2}{c|}{ ERG amplitude $(\boldsymbol{\mu V})$} \\
\hline mcd.s/m & log photons/cm $/ \mathbf{s}$ & b-wave & a-wave \\
\hline 0.03 & 7.65 & -- & -- \\
\hline 0.1 & 8.2 & -- & -- \\
\hline 0.3 & 8.65 & -- & -- \\
\hline 1 & 9.2 & $6.9 \pm 3.4$ & -- \\
\hline 3 & 9.65 & $45.9 \pm 8.8$ & -- \\
\hline 10 & 10.2 & $107.8 \pm 14.3$ & -- \\
\hline 30 & 10.65 & $154.6 \pm 15.5$ & -- \\
\hline 100 & 11.2 & $166.6 \pm 17.6$ & $20.1 \pm 4.4$ \\
\hline 300 & 11.65 & $207.8 \pm 30$ & $39.8 \pm 8.3$ \\
\hline 1000 & 12.2 & $244.1 \pm 28.3$ & $66.5 \pm 12.4$ \\
\hline 3000 & 12.65 & $264.4 \pm 31.8$ & $94.8 \pm 14.5$ \\
\hline 10000 & 13.2 & $258.7 \pm 31.8$ & $111.7 \pm 15.1$ \\
\hline 30000 & 13.65 & $286.5 \pm 33.1$ & $128.5 \pm 16.7$ \\
\hline
\end{tabular}

Table 2-7: ERG a- and b-wave amplitudes from the scotopic intensity series. 


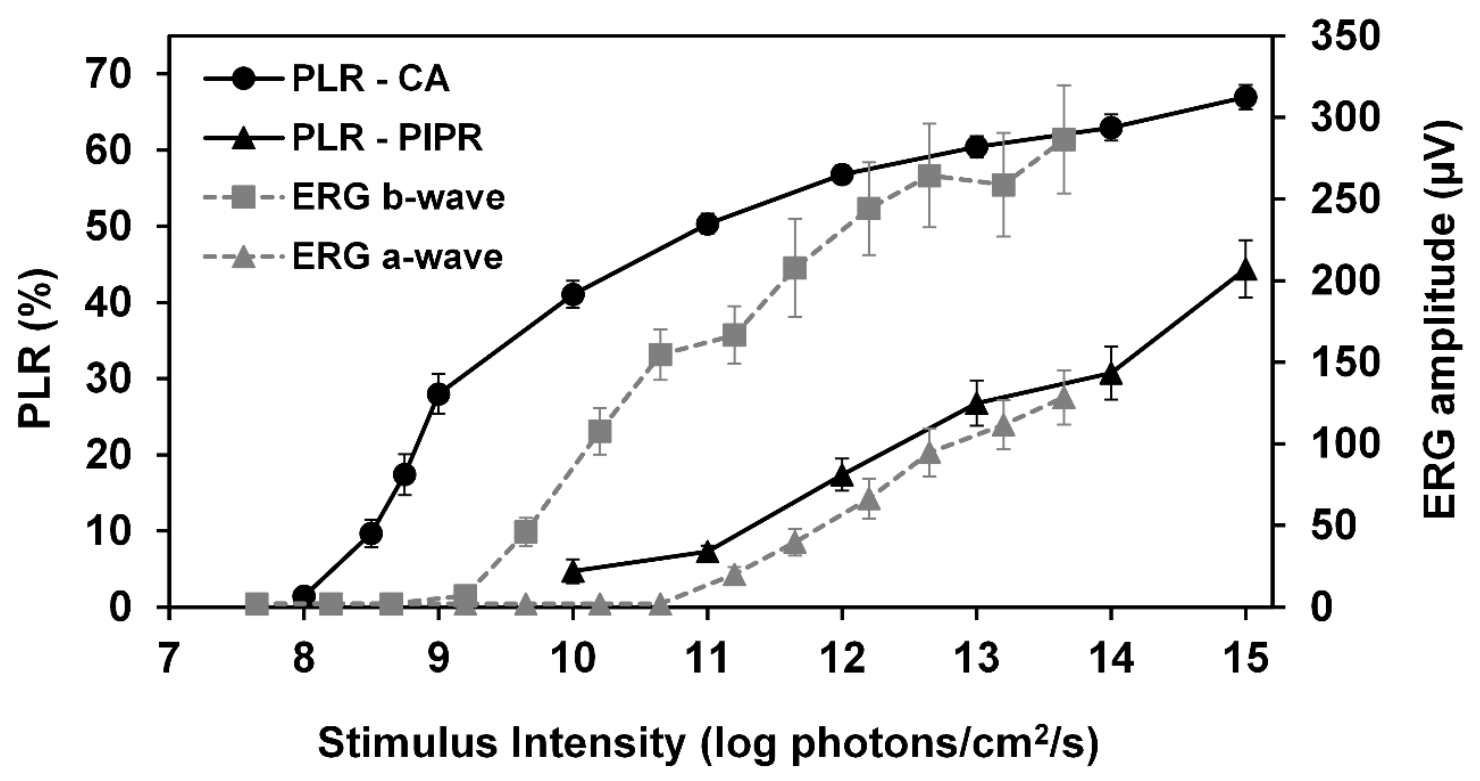

Figure 2-9: Average PLR constriction amplitude and PIPR ( $n=7)$ and ERG aand $b$-wave amplitudes $(n=5)$ in response to white light stimuli of increasing intensity. Assuming a threshold for reliable detection of the PLR at $5 \%$ pupil constriction and for the ERG b-wave an amplitude of $10 \mu \mathrm{V}$, the ERG threshold is more than 10 times higher than the PLR threshold. Pupil constriction amplitude is shown as percent of baseline pupil area. ERGs were recorded under dexmedetomidine sedation and PLRs were recorded under anesthesia with dexmedetomidine and isoflurane. Error bars represent standard error of the mean (SEM).

\subsection{Discussion}

We have developed a recording system and protocol capable of reliably quantifying the PLR in the dog that can easily be adapted for use in other species. The system provides great flexibility with respect to stimulus color, duration, and range of intensity allowing for thorough evaluation of many parameters known to affect the PLR.

Due to similarities in size and anatomy between canine and human eyes and the similarities between these species in neural pathways between the eye and 
brain, the dog is an excellent model for studying retinal-mediated responses to light stimuli (Sargan 2004; Casal and Haskins 2006) Many diseases affecting these responses in people are recapitulated in dogs (Baehr and Frederick 2009). This makes dogs very useful for evaluating potential therapeutic interventions to treat diseases of the visual system. For example, identification of a dog model of Leber congenital amaurosis and successful development of gene therapy in this model served as the basis for its subsequent use in people (Narfstrom, Katz, Bragadottir, et al. 2003; Acland et al. 2001; Hauswirth et al. 2008). In people, the efficacy of such therapeutic interventions can be assessed by asking patients how well they can see. Unfortunately, this is not possible with dogs or other animal models, and behavioral testing for visual function is often impractical. Thus, for most animal studies, assessment of visual system integrity is usually performed using ERG recordings, sometimes in conjunction with subjective observation of the PLR to a bright light stimulus. The sensitivity of the ERG performed with the recording electrode placed on the surface of the cornea is limited, due in large part to the distance between the recording electrode and the retina in which the electrical signals are generated (Bui et al. 1998; Derwent et al. 2006) As our data illustrate, the threshold for the PLR ( $8.59 \pm 0.30$ log units $)$ is much lower than that of the ERG (9.56 \pm 0.20 log units). In addition, people and dogs with non-recordable ERG responses can still retain useful vision and exhibit a PLR (Melillo et al. 2012). Consequently, quantitative PLR measurements may 
be able to detect useful effects of therapeutic interventions that are too small to be detected with the ERG.

It should be noted that the ERG and PLR were recorded under different form of chemical restraint. The conditions used for ERG recordings were those that have been well established and are currently in widespread use in veterinary clinical practice and research. It is unlikely that the current standards for chemical restraint used for ERG recordings will be modified. In addition, ERGs recorded in dogs under general anesthesia with isoflurane are greatly reduced in amplitude compared with ERGs recorded in the same dogs under sedation with dexmedetomidine (Lin et al. 2009). Based on these considerations, we attempted to obtain the PLR recordings using methods of chemical restraint similar to that which is used widely for ERG recordings. Unfortunately, as illustrated in our study, using the sedation protocol employed for ERG recordings, we were unable to obtain a stable baseline pupil size. As a result, different protocols for chemical restraint will need to be used for ERG and PLR recordings in both research and clinical settings. Given this limitation, our conclusions about the differences in thresholds between the ERG and PLR are valid for the conditions under which these recordings can practically be made under standardized conditions.

Quantitative assessment of the PLR will enable sensitive detection of defects within the neural pathway that mediates this reflex. However, PLR recordings alone will not pinpoint the causes of observed deficits. Accurate interpretation of 
the PLR data will require concurrent assessment of retinal function. If retinal responses to light are abnormal, identification of defects in parts of the PLR pathway downstream from the retina will be difficult to identify. However, if retinal function is normal, PLR abnormalities can be attributed to neurological pathology outside of the retina. With animal models it will be possible to associate PLR alterations with neuroanatomical localization of pathology.

For large animal recording, the system described here has several advantages over previously described methods for PLR assessment. The protocol we developed allows for stable positioning of the eye and full visualization of the pupil at all times. The average baseline pupil diameter achieved under anesthesia was similar to that reported in another canine study performed under medetomidine sedation (Grozdanic et al. 2007). However, the maximum pupil constriction achieved under medetomidine sedation was only $22 \%$ with a 0.2 second stimulus of $57 \mathrm{~cd} / \mathrm{m}^{2}\left(285 \mathrm{~cd} . \mathrm{s} / \mathrm{m}^{2}\right.$ or $\sim 14.6 \mathrm{log}$ photons $/ \mathrm{cm}^{2} / \mathrm{s}$ ). In contrast, the protocol described here regularly achieved pupil constriction of greater than $60 \%$ using a comparable stimulus intensity. This constriction amplitude is similar to values achieved in awake human subjects using an analogous recording system and stimulus (Fan et al. 2009b).

Melanopsin, the ipRGC photopigment, has peak sensitivity at $480 \mathrm{~nm}$, and these cells are slow to activation and have a high threshold (Gamlin et al. 2007). Below the ipRGC threshold, PLRs to blue and red light have been shown to be quite similar (Markwell et al. 2010). However, when the ipRGCs are strongly 
activated they cause a sustained pupil constriction persisting after light offset that is not seen with a red stimulus outside the melanopsin spectral sensitivity (Kankipati, Girkin, and Gamlin 2010; Markwell, Feigl, and Zele 2010). We were able to replicate these findings (Figure 2-8) suggesting that these characteristics of melanopsin input to the PLR are conserved in the dog.

The current study demonstrated a large difference between the use of sedation, neuroleptanalgesia, and dissociative anesthesia compared to general anesthesia with respect to baseline pupil size and spontaneous pupil fluctuation. Obtaining reliable PLR recordings using injectable chemical restraint alone was not possible due to spontaneous fluctuations in pupil size and movement of the eyes. For a person or animal in a relaxed state with a fixed visual focus, the pupil typically reaches a steady size determined by the balance of input from the sympathetic and parasympathetic systems (Lowenstein and Lowenfeld 1950). The observed pupil fluctuation therefore indicates variable input from these two systems. This instability may also reflect the fact that systemic drug levels change continuously after intramuscular administration (Dyck et al. 1993). In contrast, the continuous delivery of isoflurane allowed greater control over the level of anesthesia and resulted in a stable pupil size that was maintained over the period necessary to complete the stimulus protocol. In human PLR studies isoflurane reduced the baseline pupil size and significantly depressed the constriction amplitude of the PLR (Larson et al. 1991). As the concentration of isoflurane was increased, pupil constriction was further suppressed with no 
detectable PLR at 1\% end-tidal isoflurane (Larson et al. 1991). While we did observe a reduction in baseline pupil size relative to unsedated dogs, we regularly observed large PLR constriction amplitudes. This difference from the human study could be due to the differences in the duration of anesthesia, which has been shown to affect pupil size and responsiveness (Tayefeh et al. 1997). While our PLR measurements were completed within 1.5 hours of initiating isoflurane, the human study measured the PLRs 3-5 hours after initiating isoflurane. In addition, the PLRs observed in anesthetized dogs were very similar to those recorded in people who were not chemically restrained (Fan et al. 2009a). Under the conditions of this investigation, general anesthesia using isoflurane did not appear to significantly alter the canine PLR.

The pupillography system used in these studies was constructed with readily available components and could be assembled by research laboratories or could be commercialized for application in clinical veterinary ophthalmology and neurology. User control of stimulus characteristics and recording parameters allows great flexibility in probing various aspects of the visual system involved in the PLR and allows the system to be adapted for use in a variety of species. Varying the stimulus parameters also allows for the evaluation of relative contributions of the different retinal chromophores in initiating this reflex (Kankipati, Girkin, and Gamlin 2010; Markwell, Feigl, and Zele 2010). Objective PLR analysis could also aid in localizing disease-related abnormalities in neurological and ophthalmic disorders (Park et al. 2011). 


\section{CHAPTER 3}

\section{PLR DEFICITS IN A CANINE MODEL OF CLN2}

\subsection{Introduction}

Childhood-onset neuronal ceroid lipofuscinoses (NCLs) are autosomal recessively inherited lysosomal storage disorders characterized by progressive vision loss culminating in blindness, cognitive and motor decline, and seizures (Haltia and Goebel 2012; Mole, Williams, and Goebel 2011) The NCLs are uniformly fatal. Clinical signs of NCL result from wide-spread progressive neurodegeneration that is accompanied by intracellular accumulation of autofluorescent storage bodies in nervous and other tissues, including the retina (Haltia 2006). Currently, several approaches to therapy are under investigation, including gene and stem cell therapies in addition to direct enzyme replacement (Pierret, Morrison, and Kirk 2008; Hobert and Dawson 2006; Souweidane et al. 2010).

Development of effective therapeutic interventions will be facilitated by the availability of suitable animal models. Naturally-occurring NCLs have been identified in several larger animal species, including dogs (Awano et al. 2006a; Awano et al. 2006b; Bond et al. 2013; Farias et al. 2011; Katz et al. 2005; Katz et al. 2011; O'Brien and Katz 2008; Palmer et al. 2011; Sanders et al. 2010). Among the canine NCLs is a form of the disease in Dachshunds which results from a null mutation in TPP1 that encodes the lysosomal enzyme tripeptidylpeptidase-1 (TPP1) (Awano et al. 2006b) Human subjects with mutations in 
TPP1 (CLN2) have a form of NCL (CLN2) in which neurological signs typically first appear between 2 and 4 years of age and progress to death, usually by the middle teenage years (Haltia and Goebel 2012; Mole, Williams, and Goebel 2011). Dachshunds that are homozygous for the TPP1 null mutation develop neurological signs similar to those observed in children with CLN2 and reach end stage disease between 10 and 11 months of age (Vuillemenot et al. 2011) The retinal pathology associated with canine CLN2 has been previously described in a small group of Dachshunds (Katz et al. 2008). Affected dogs exhibit marked deficits in ERG b-wave amplitude and significant thinning of the inner retina by disease end-stage (Katz et al. 2008).

The ERG is widely used to assess retinal function in animals in which it is difficult to evaluate vision. However, the ERG is relatively insensitive, and subjects with profoundly depressed ERG responses can retain significant visually mediated behavior (Acland et al. 2001; Melillo et al. 2012; Narfstrom et al. 2003a; Narfstrom et al. 2003b). In addition, the ERG only measures responses of the retina itself to light stimuli and provides no information on the transmission of light-induced neural signals to the brain. The pupillary light reflex (PLR) depends on both retinal function and the intactness of central neural pathways involved in mediating this response to light. In addition, the PLR can be elicited with significantly dimmer stimuli than can the ERG (Whiting et al. 2013; Yao et al. 2006). Therefore, quantitative evaluation of the PLR can be used in conjunction with the ERG as a sensitive tool to evaluate the integrity of the entire complex 
network of neuronal circuitry involved in modulating pupil size (Park et al. 2011; F. Fotiou et al. 2000). Such a tool will be particularly useful in characterizing diseases such as CLN2 that involve pathological changes in both the retina and other areas of the central nervous system. In these diseases, ideal therapeutic interventions would ameliorate both retinal and central nervous system signs and would therefore preserve both the ERG and the PLR. In light of therapeutic studies currently under way with the Dachshund model of CLN2, studies were undertaken to determine whether the PLR is affected in this model and to assess the relationship of any changes in the PLR to disease-related ERG alterations.

\subsection{Methods}

\subsubsection{Animals}

Studies employed purpose-bred long-haired miniature Dachshunds housed in a research facility at the University of Missouri. Dogs were entrained to a 12:12 daily light cycle and were socialized daily in addition to receiving routine husbandry care. Dogs were genotyped at the TPP1 locus using an allelic discrimination assay and an Applied Biosystems real time PCR instrument (Applied Biosystems, Carlsbad, CA). Dogs utilized in this study were either homozygous normal $(T P P 1+/+)(n=14)$ or homozygous for the mutant allele (TPP1 -/) $(\mathrm{n}=9)$. Heterozygous carriers of the TPP1 mutation (TPP1 -/+) were

used for breeding but were not evaluated for responses to light stimuli. Seven of the normal and three of the affected dogs had an intraventricular (ICV) catheter 
placed as part of another study. Four of the normal dogs received infusions of recombinant TPP1 protein, which should not have any effect on the PLR in a genetically normal dog that already produces this protein (Vuillemenot et al. 2013). The remaining dogs with implanted ICV catheters received infusions of artificial CSF, which is an inert substance. Infusions were repeated every 2 weeks starting between 2 and 3 months of age (Vuillemenot et al. 2013). All studies were performed in compliance with the ARVO Statement for the Use of Animals in Ophthalmic and Vision Research and were approved by the University of Missouri Animal Care and Use Committee.

\subsubsection{Ophthalmic Examinations}

Prior to inclusion in the study, all dogs received a complete ophthalmic examination, at 10-12 weeks of age. Assessment of the globe at this age precedes any signs of retinal or neurological impairment due to CLN2 in the affected dogs (Katz et al. 2008). Any dogs with evidence of visual compromise or other ophthalmic conditions deemed threatening to vision were excluded from the study $(n=1)$. Examinations were repeated monthly for CLN2-affected dogs and every other month for genetically normal dogs. Examinations included visually-mediated behavioral assessment and slit lamp biomicroscopy (SL14; Kowa Co. Ltd., Tokyo, Japan). Pupils were dilated with a short-acting mydriatic (tropicamide 1\%; Alcon, Fort Worth, TX), and indirect ophthalmoscopy (12500, Welch Allyn Inc., Skaneateles Falls, NY, USA) was performed. Fundus photographs were taken following each examination (NM-100; Nidek Co. Ltd., 
Freemont, CA) and archived electronically. Photos were reviewed to evaluate for small changes in retinal appearance.

\subsubsection{Electroretinography}

Beginning at 3 months of age, bilateral ERG evaluations were performed monthly as previously described (Katz et al. 2008). For ages at which dogs underwent PLR assessment, ERG evaluation was performed within one week after the PLR recording session. ERGs were bilaterally elicited and simultaneously recorded with a portable unit (HMsERG model 2000; RetVet Corp., Columbia, MO). The right and left mini-ganzfeld domes were positioned approximately $2 \mathrm{~cm}$ from the corresponding eye. Dogs were deeply sedated with intramuscular administration of dexmedetomidine (30 - $40 \mu \mathrm{g} / \mathrm{kg})$ and prepared for the ERG session in ordinary room light. Ketamine (up to $5 \mathrm{mg} / \mathrm{kg}$ ) was used in combination with the dexmedetomidine for dogs 6 months of age and younger to achieve adequate sedation. It was omitted in older dogs to reduce the risk of seizure associated with CLN2, and it was no longer necessary for adequate sedation with this age group.

Each ERG session consisted of scotopic and photopic ERGs in accordance with the Dog Diagnostic Protocol, recommended by the European College of Veterinary Ophthalmology, primarily for evaluation of rod and cone function (Narfström et al. 2002). During 20 minutes of dark adaptation, scotopic lowintensity rod responses were elicited every 4 minutes at a stimulus intensity of 
$10.2 \log$ photons $/ \mathrm{cm}^{2} / \mathrm{s}\left(0.01 \mathrm{~cd} . \mathrm{s} / \mathrm{m}^{2}\right)$. Thereafter, scotopic responses were elicited using flashes of 12.65 and 13.2 log photons $/ \mathrm{cm}^{2} / \mathrm{s}\left(3 \mathrm{~cd} . \mathrm{s} / \mathrm{m}^{2}\right.$ and 10 $c d . s / \mathrm{m}^{2}$ ) to evaluate mixed rod and cone function. The eyes were then exposed to diffuse white light at a luminance of $13.65 \log$ photons $/ \mathrm{cm}^{2} / \mathrm{s}\left(30 \mathrm{~cd} . \mathrm{s} / \mathrm{m}^{2}\right)$ for 10 minutes immediately after which responses to single $12.65 \mathrm{log}$ photons $/ \mathrm{cm}^{2} / \mathrm{s}$ $\left(3 \mathrm{~cd} . \mathrm{s} / \mathrm{m}^{2}\right)$ flash stimuli were recorded. This was immediately followed by evaluation of responses to $30-\mathrm{Hz}$ photopic flicker stimuli at the same light intensity. ERG waveforms in all recordings were evaluated, and the amplitudes and implicit times for the a- and b-waves were measured as previously described (Marmor et al. 2004).

\subsubsection{PLR Recordings}

The PLR was recorded in each dog at 4, 6, 8, 10, and 12 months of age. CLN2-affected dogs were typically euthanized at 10 to 11 months of age due to progression of neurologic deficits, so the 12 month recordings were not obtained in these dogs. The 10 month recording was not obtained in three of the affected dogs due to the need for early euthanasia prior to 10 months of age. All recordings were done during the light period of the daily $12: 12$ light-dark cycle. The detailed methods for obtaining the PLRs have been described previously (Whiting et al. 2013). Dogs were kept in dim light (0.9 lux) for at least 1 hour, including preparation time, and in complete darkness for 10 minutes prior to recording. After 30 minutes of dim light adaptation, dogs were pre-medicated with dexmedetomidine (20-25 $\mathrm{\mu g} / \mathrm{kg}$ IM) prior to induction of anesthesia with 
propofol (IV to effect, $1.49 \pm 0.59 \mathrm{mg} / \mathrm{kg}$ (mean \pm SD); PropoFlo 28, Abbott Laboratories, Abbott Park, IL). Dogs were intubated with a cuffed endotracheal tube and anesthesia maintained with isoflurane (1.5\% vaporizer setting; Terrell, Piramal Healthcare, Boise, ID) in oxygen. The dog was positioned in sternal recumbency, with the head placed on a deflatable cushion. The cornea of the right eye was anesthetized with proparacaine and a lid speculum was inserted to ensure that the nictitating membrane and eyelids did not interfere with light exposure or visualization of the pupil. In addition, a small stay suture was placed in the bulbar conjunctiva on the central axis approximately $5 \mathrm{~mm}$ superior to the limbus to facilitate globe manipulation to maintain centration of the pupil on the optical axis of the recording apparatus. The eye was regularly lubricated with saline eye wash solution throughout the procedure.

Recordings were performed with a custom apparatus (Fan et al. 2009b) capable of timed delivery of a visible light stimulus from a mounted high-power LED (MCWHL2; Thorlabs Inc., Newton, NJ) and concurrent recording of pupil images at 30 frames per second using an infrared-sensitive camera (PC164CEX2; Supercircuits Inc., Austin, TX) and continuous infrared illumination (880 nm LED) for visualization of the eye. The direct PLR of the right eye was evaluated using a standardized protocol of 100 millisecond flashes of white light at each of 10 intensities between 8 and $15 \log$ photons $/ \mathrm{cm}^{2} / \mathrm{s}(0.1$ to 1400 lux).

Pupil images were analyzed using the batch processing feature in Photoshop (Adobe Systems Inc; San Jose, CA). A list of image frame number and 
corresponding pupil area was exported to a spreadsheet and used to calculate desired parameters including latency, constriction amplitude, and velocity of constriction and redilation. Area measurements were converted from pixels to $\mathrm{mm}^{2}$ based on the known size of the lid speculum present in each pupil image.

\subsubsection{PLR Parameters}

The PLR parameters that are typically considered are illustrated in Figure 1-4. For the studies described here, baseline pupil diameter is the average pupil diameter in a dark-adapted dog, over a 1-second period before the light stimulus. PLR constriction amplitude is defined as the difference between baseline pupil diameter and minimum pupil diameter attained following the light stimulus. Latency is defined as the time between stimulus onset and the beginning of pupil constriction. Average constriction velocity is calculated as the constriction amplitude divided by the constriction time, where constriction time is calculated between the beginning of pupil constriction and the minimum pupil diameter. Redilation of the pupil is biphasic with a fast initial redilation and slower secondary redilation. Average redilation velocity is a measure of initial redilation and is defined as half the constriction amplitude divided by the time required for the pupil to redilate from its minimum diameter to half the baseline pupil size. For the brightest flash (15 log photons $/ \mathrm{cm}^{2} / \mathrm{s}$ ), the rate of secondary redilation was calculated for the period from 15 to 85 seconds after light offset. 


\subsubsection{Statistical Analysis}

All statistical tests were performed using SigmaPlot (Systat Software Inc., San Jose, CA). Data were subjected to the Shapiro-Wilk test to confirm normal distribution. One-way analysis of variance (ANOVA) was used to test for any differences between the normal dogs that were untreated, those that received artificial CSF, and those that received TPP1 enzyme. No difference was found amongst these groups, so all data from genetically normal dogs were combined to serve as the normal control group.

Repeated measures 2-way ANOVA was performed with disease status and age as the two factors. This test was used to determine if significant differences exist between the normal control group and the CLN2-affected group with respect to any of the calculated PLR parameters for a given stimulus intensity. This test also determined whether age had a significant effect on PLR parameters or if there was any significant interaction between age and disease status. Follow-up pair-wise comparisons were performed with the Holm-Sidak correction $(\alpha=0.05)$ to control family-wise error rate. The Pearson productmoment correlation coefficient was also calculated to test for correlation amongst the PLR parameters of latency, constriction velocity, constriction amplitude, and redilation velocity.

The same statistical methods were used to evaluate ERG parameters to determine whether differences exist between normal and affected dogs and whether age was significant factor. 


\subsubsection{Fluorescence Microscopy}

The neural pathway involved in mediating the PLR in the dog includes the pretectal nucleus and the parasympathetic neurons of the oculomotor nucleus, so disease-related changes in these would be expected to affect the PLR. Therefore, analyses were performed to determine whether these neurons exhibit the accumulation of autofluorescent storage material that is characteristic of the NCLs.

Regions of the brain containing these neurons were collected shortly after euthanasia and incubated in $0.1 \%$ glutaraldehyde, $3.5 \%$ paraformaldehyde, 0.13 M sodium cacodylate, and $0.1 \mathrm{mM} \mathrm{CaCl} 2(\mathrm{pH} 7.4)$ with gentle agitation for approximately 24 hours. The samples were then incubated in $0.17 \mathrm{M}$ sodium cacodylate, $\mathrm{pH} 7.4$ with gentle agitation for at least 1 hour, embedded (TissueTek; Sakura Finetek, Torrance, CA), and frozen on dry ice.

Five micrometer thick sections were cut from the Tissue Tek-embedded samples and mounted on glass slides (Super Frost; Fisher Scientific, Pittsburgh, $\mathrm{PA}$ ) in $0.17 \mathrm{M}$ sodium cacodylate. The sections were examined with a microscope (Axiophot; Carl Zeiss Meditec, Inc., Dublin, CA) equipped with epiillumination from a high-pressure, 50-W mercury vapor lamp, a 40X objective lens (Plan-Neofluar) with a 1.3 numerical aperture, a 395- to 440-nm band-pass exciter filter, a chromatic beam splitter (FT 460), and an barrier filter (LP 515; all from Carl Zeiss Meditec, Inc.). Photography was performed with an Olympus DP72 digital camera. 


\subsection{Results}

\subsubsection{Ophthalmic Examinations}

Initial examination in all dogs revealed normal adnexal and ocular structures. Some affected mutant (TPP1 -/-) dogs developed progressive multifocal retinal detachment lesions consistent with CLN2 between the ages of 5 and 10 months $(n=6)$ (Pearce et al. 2012). Other affected dogs retained phenotypically normal fundus exams throughout the study period $(n=3)$. Homozygous normal (TPP1 $+/+$ ) dogs had consistently normal ophthalmic examinations throughout the study period.

\subsubsection{ERG}

The ERG a-wave was relatively well preserved in CLN2-affected dogs (Figure 3-1, Figure 3-2). Scotopic recordings did begin to show a decrease in a-wave amplitude at 8 months of age, and the difference between normal and affected dogs was statistically significant at 10 months of age (Figure 3-1, Figure 3-2A). CLN2-affected dogs exhibited substantial deficits in the b-wave amplitude of both scotopic and photopic recordings (Figure 3-1, Figure 3-2). Significant deficits in b-wave amplitude from rod recordings were present at 3 months of age. By 6-7 months of age, the rod response b-wave was non-recordable in affected dogs after 20 minutes of dark adaptation (Figure 3-2C). Scotopic recordings reflecting mixed input from rods and cones illustrated a progressive decrease in b-wave amplitude, with significant deficits beginning at 5 months of age (Figure 3-2D). 
Cone-mediated b-wave deficits were statistically significant beginning at 4 months of age and deficits continued to progress with age, though cone responses remained recordable through 10 months of age (Figure 3-2E). Cone inner retinal function, as illustrated by the b-wave amplitude of photopic flicker recordings, was significantly reduced in CLN2-affected dogs beginning at 5 months of age. While the deficits in flicker-induced b-wave amplitude persist through end-stage disease, this response remained recordable through 10 months of age (Figure $3-2 \mathrm{~F})$. 

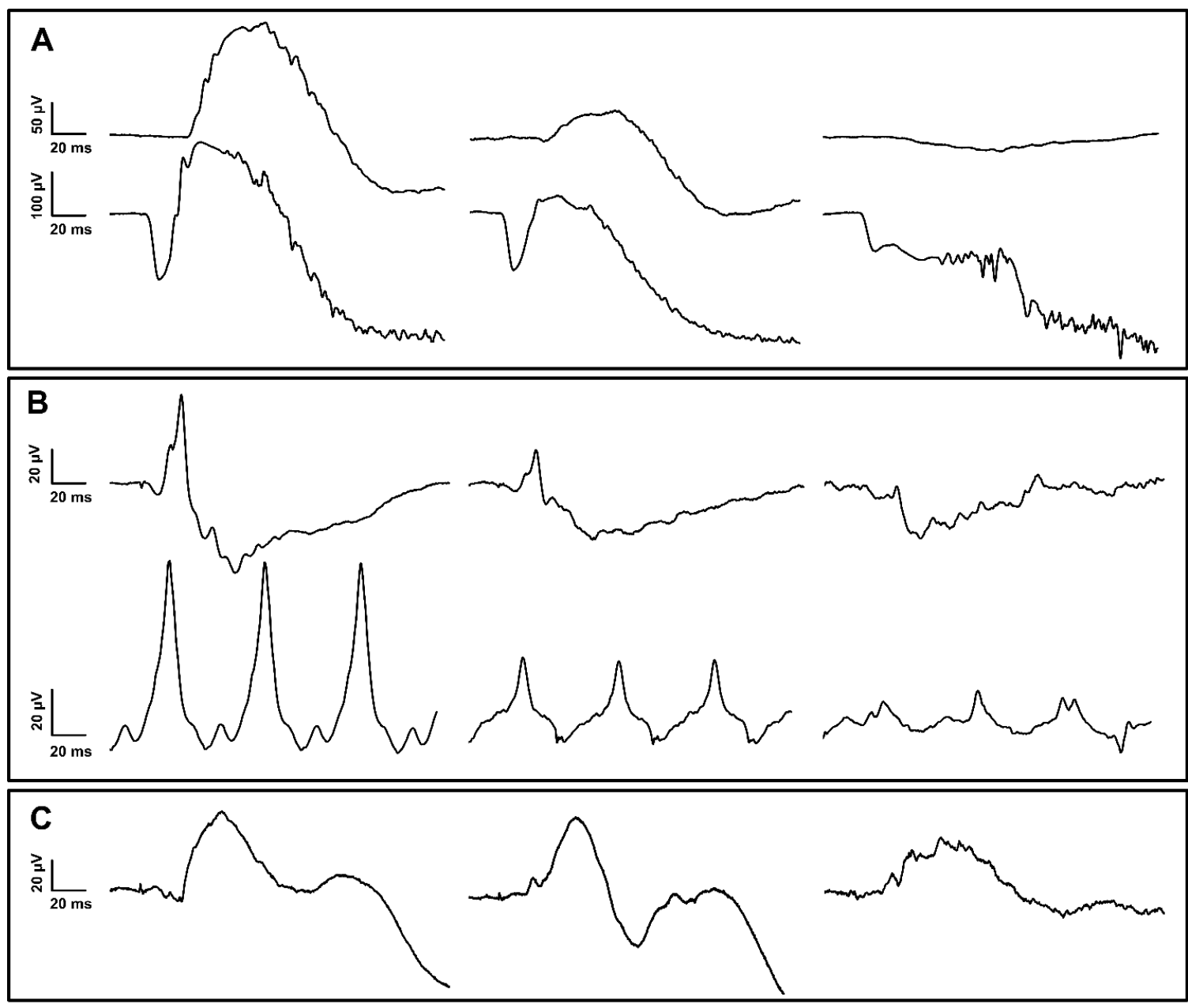

Figure 3-1: Representative ERG \& VEP tracings from a 10 month old normal dog (left), 6 month old affected dog (middle) and 10 month old affected dog (right). (A) Scotopic rod recordings and $10 \mathrm{~cd} . \mathrm{s} / \mathrm{m}^{2}$ mixed rod and cone recordings. (B) Photopic cone single flash and $30 \mathrm{~Hz}$ flicker recordings. (C) VEP recordings performed on light-adapted dogs. 

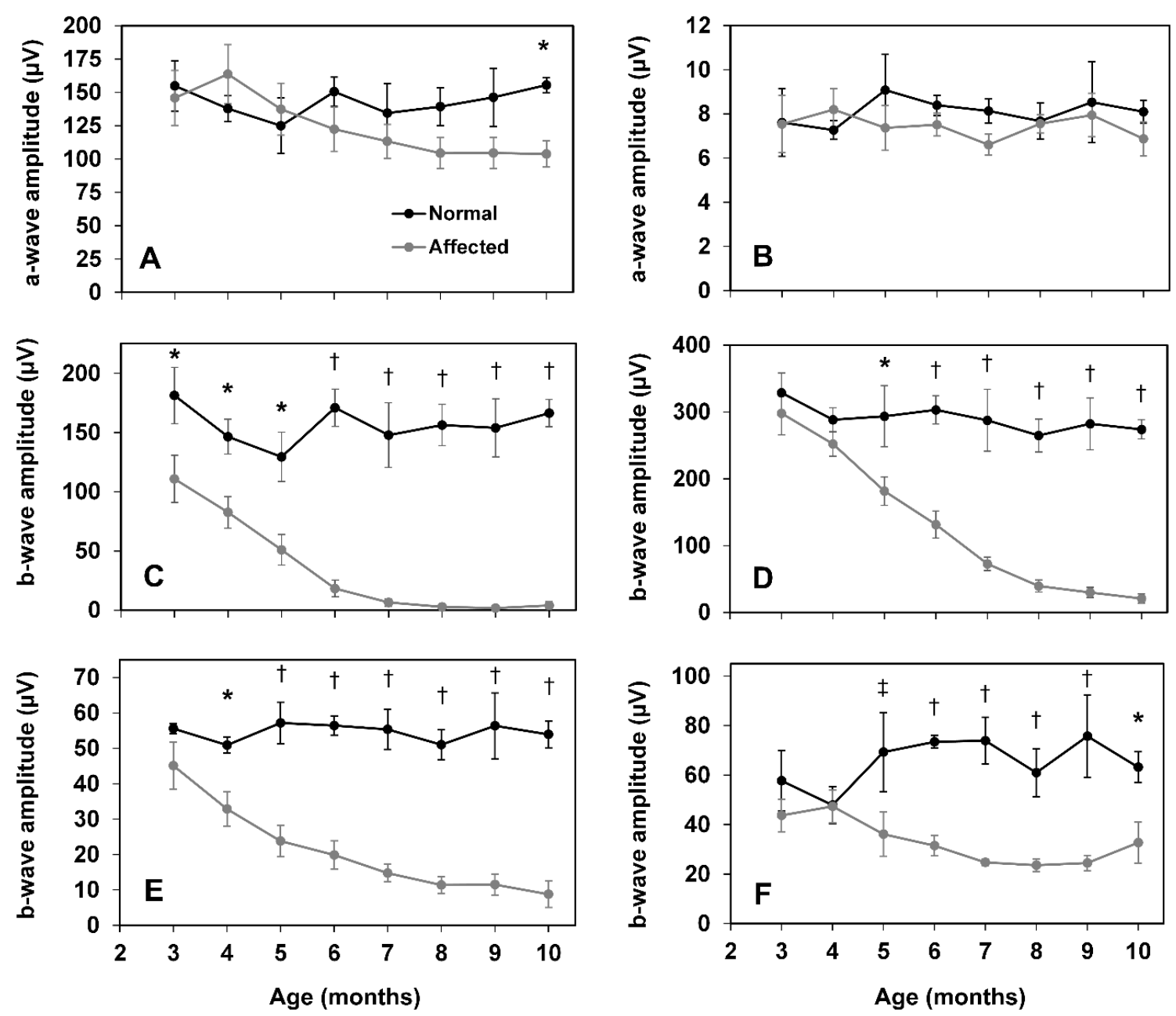

Figure 3-2: $E R G$ amplitudes as a function of age in normal and CLN2-affected dogs. ERG a-wave amplitude from $10 \mathrm{~cd} . \mathrm{s} / \mathrm{m}^{2}$ scotopic (A) and photopic (B) recordings. Significant deficits in a-wave amplitude are not present until 10 months of age and only with scotopic recordings. ERG $b$-wave amplitude from scotopic rod responses (C), scotopic mixed responses from rods and cones at 10 cd.s $/ \mathrm{m}^{2}(\mathrm{D})$, and photopic cone (E) and $30 \mathrm{~Hz}$ flicker recordings (F). Significant deficits in b-wave amplitude exist even in early stages of disease $(\dagger, p<0.001$; *, $p<0.005 ; \ddagger, p<0.05)$. Error bars represent SEM.

Results of the VEPs recordings were quite variable in both normal and affected dogs. No statistical differences were found between normal and affected dogs. 


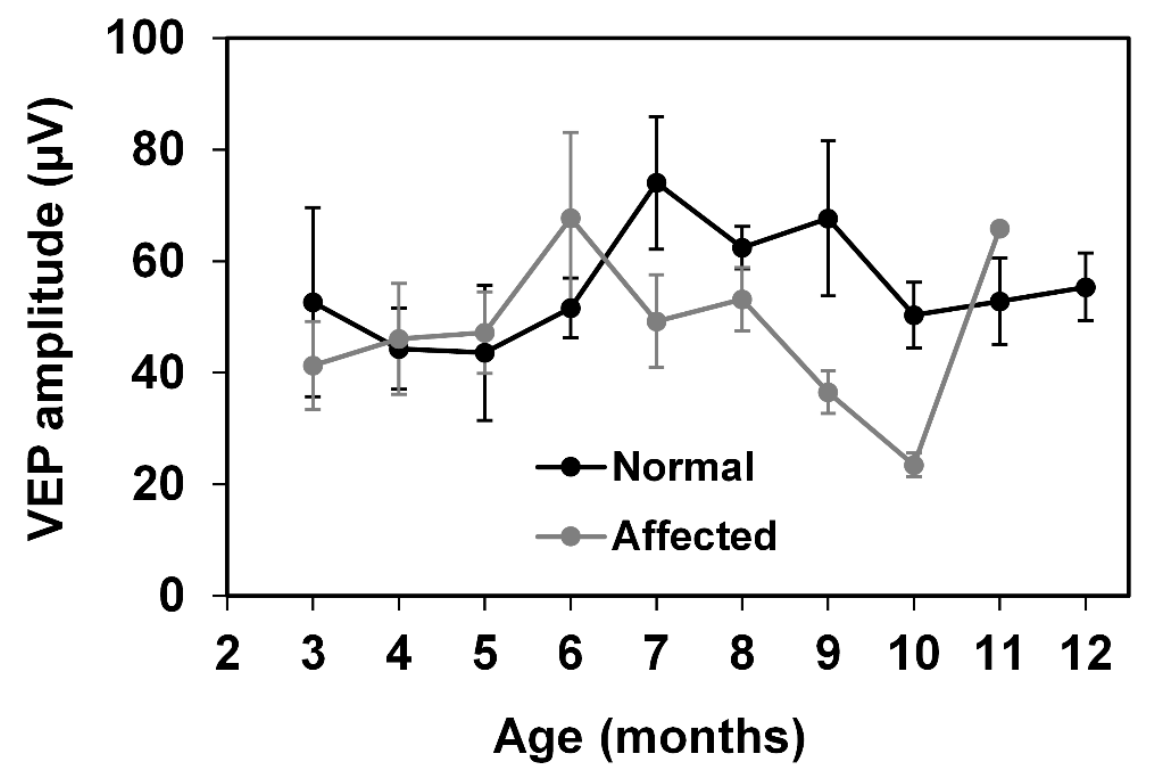

Figure 3-3: VEP amplitude as a function of age in normal and CLN2-affected dogs. Results were variable with no statistical differences between normal and affected dogs. Data at 11 months of age reflects a single affected dog.

\subsubsection{PLR}

No significant difference was found in baseline pupil diameter between normal dogs, $8.12 \pm 0.15 \mathrm{~mm}$ and CLN2-affected dogs 8 months of age or younger, $8.13 \pm 0.16 \mathrm{~mm}$ (mean $\pm \mathrm{SEM})$. However, 10 month old CLN2-affected dogs had a significantly smaller $(p<0.01)$ baseline pupil diameter than normal, $6.97 \pm 0.40 \mathrm{~mm}$ (Figure 3-4). Normal dogs showed no age-related changes in any of the evaluated PLR parameters. For both normal and affected dogs, there was a significant correlation between PLR constriction amplitude and each other parameter including latency, constriction velocity, and initial redilation velocity with correlation coefficients $(r)$ greater than $0.8(p<0.00001)$. 
PLR constriction amplitude was reduced in CLN2-affected dogs compared with that of normal dogs for stimuli between 8.5 and $13 \log$ photons $/ \mathrm{cm}^{2} / \mathrm{s}$ (Figure 3-5, Figure 3-6) when data were combined for dogs of all ages. For stimuli between 9 and 11 log photons $/ \mathrm{cm}^{2} / \mathrm{s}$, constriction amplitude was already reduced from normal by 6 months of age $(p<0.03)$ and further reduced at 8 months $(p<0.02)$ and 10 months $(p<0.001)$ of age. With a stimulus of $12 \log$ photons $/ \mathrm{cm}^{2} / \mathrm{s}$, constriction amplitude was significantly reduced beginning at 8 months of age. For stimuli of $8.5,8.75$, and $13 \log$ photons $/ \mathrm{cm}^{2} / \mathrm{s}$, the group of affected dogs as a whole was significantly reduced from the normal group with respect to constriction amplitude $(p<0.02)$, but age was not a significant factor for these intensities. In addition, constriction amplitude from 10 month old CLN2affected dogs was significantly reduced from that of 4 and 6 month old dogs for stimuli between 10 and $12 \log$ photons $/ \mathrm{cm}^{2} / \mathrm{s}(\mathrm{p}<0.001)$, and reduced from that of 8 month old dogs with a stimulus of $10 \log$ photons $/ \mathrm{cm}^{2} / \mathrm{s}(p<0.001)$. 


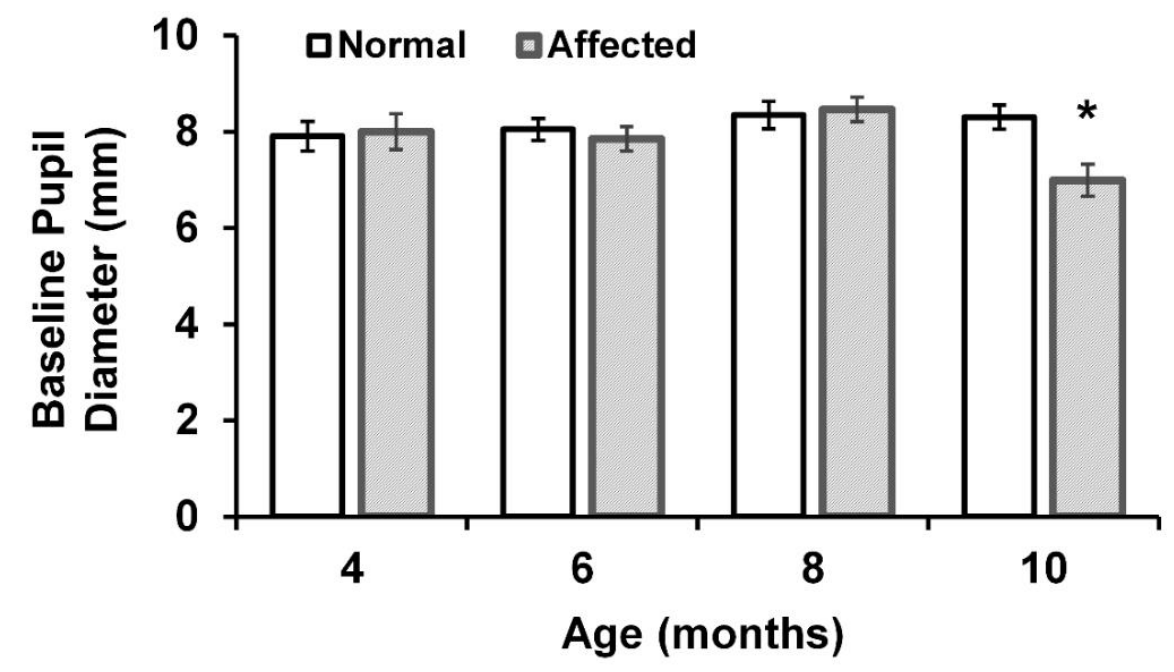

Figure 3-4: Baseline pupil diameter in anesthetized normal and CLN2-affected Dachshunds between 4 and 10 months of age. Baseline diameter is significantly reduced in 10 month old affected dogs $\left({ }^{*}, p<0.01\right)$. 


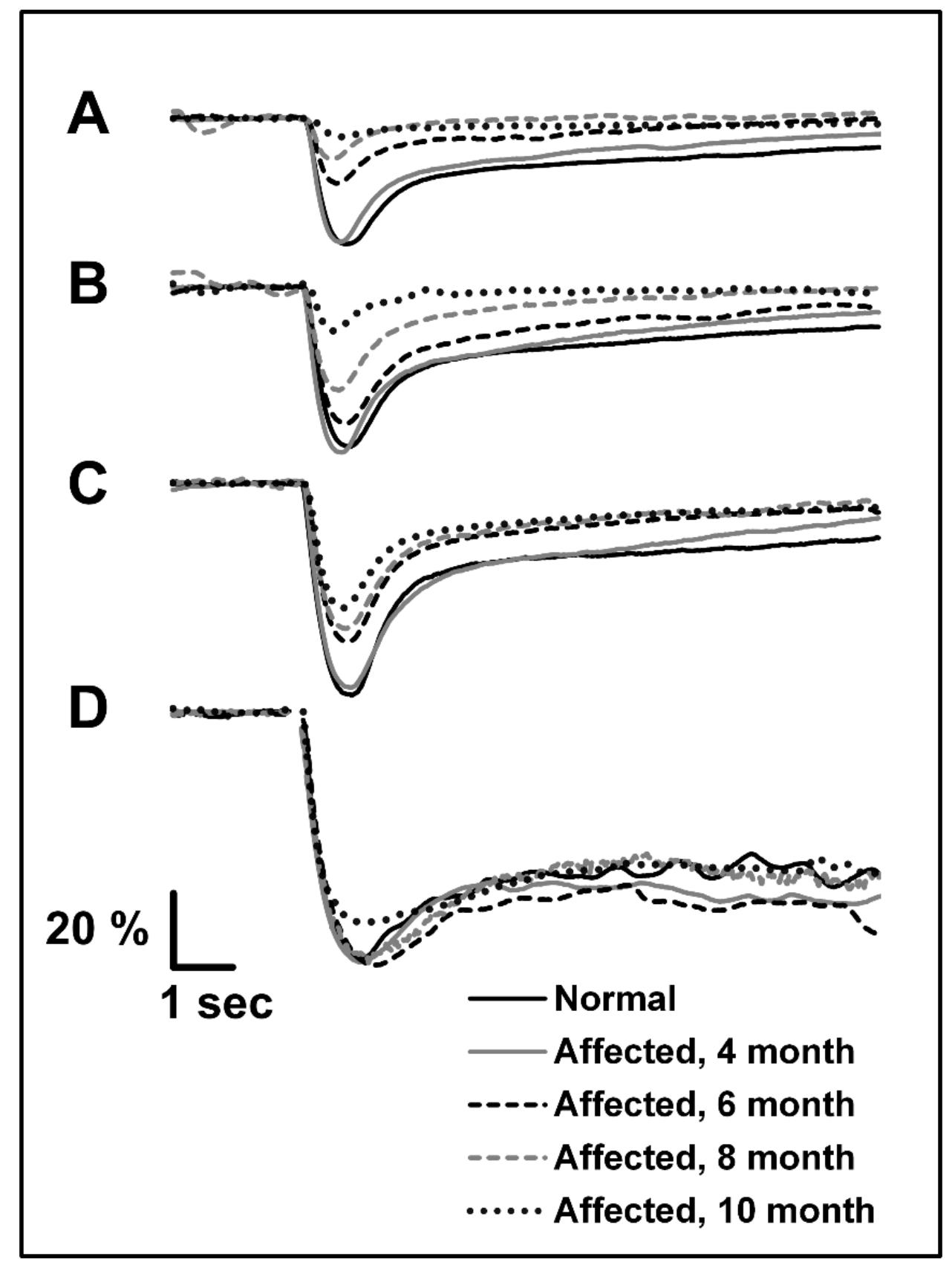

Figure 3-5: Representative PLR traces from a normal dog at 10 months of age and a CLN2-affected dog between 4 and 10 months of age. Pupil responses are shown as percent of baseline pupil area. PLRs are in response to stimuli of 9 , 10,11 , and $15 \log$ photons $/ \mathrm{cm}^{2} / \mathrm{s}$ (A-D, respectively). 


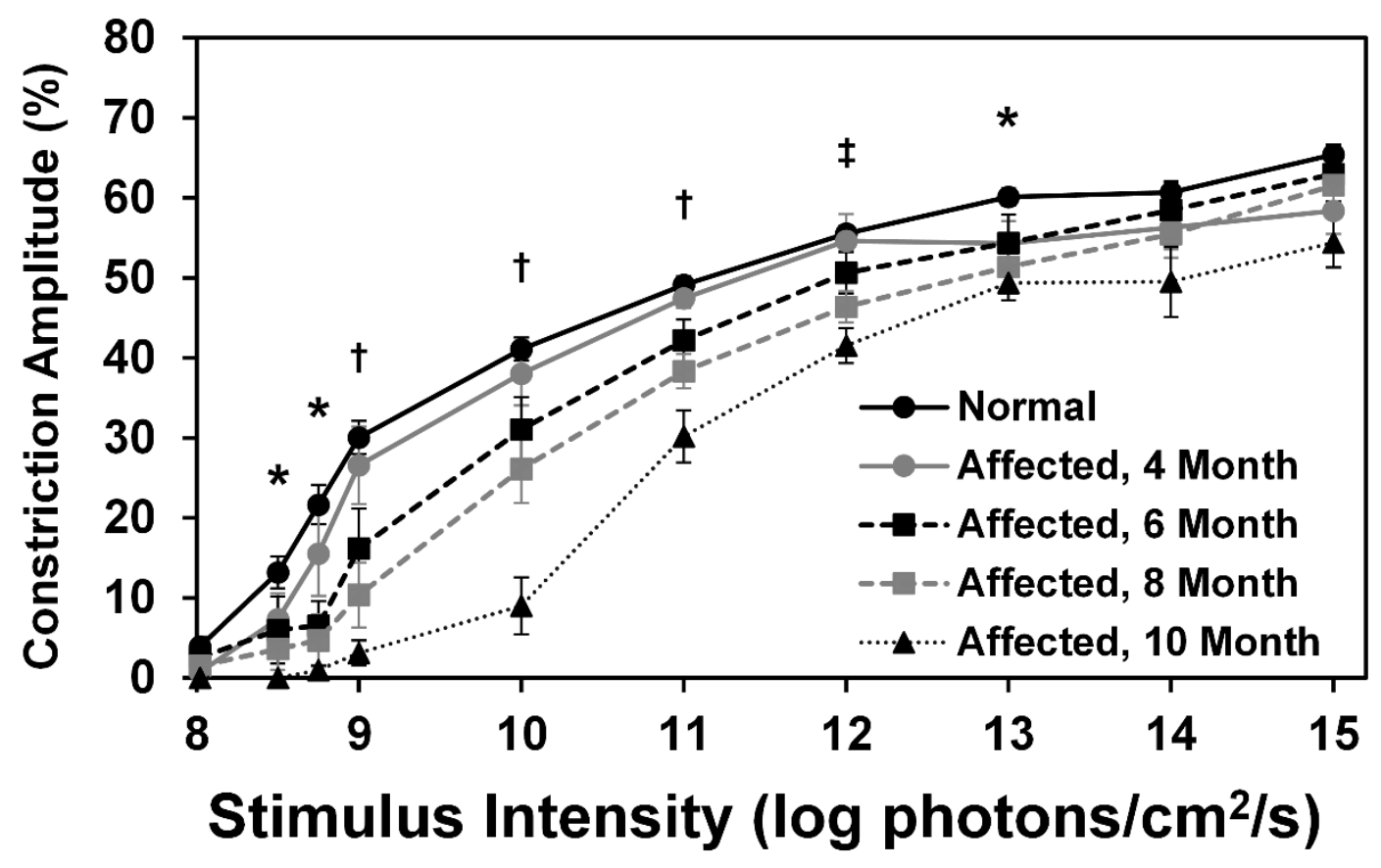

Figure 3-6: PLR constriction amplitude as a function of stimulus intensity in normal and CLN2-affected dogs. Amplitudes from normal dogs include combined data from dogs of all ages examined. For stimulus intensities between 9 and 11 log photons $/ \mathrm{cm}^{2} / \mathrm{s}$, constriction amplitude of affected dogs was progressively reduced from normal; differences were significant beginning at 6 months of age $(\dagger, p<0.03)$. With a stimulus of $12 \log$ photons $/ \mathrm{cm}^{2} / \mathrm{s}$, constriction amplitude was not significantly reduced until 8 months of age ( $\neq$, $\mathrm{p}<0.02$ ). Age was not a significant factor for stimuli of $8.5,8.75$, and $13 \mathrm{log}$ photons $/ \mathrm{cm}^{2} / \mathrm{s}$, but constriction amplitude of the CLN2-affected group as a whole was significantly reduced from normal $\left({ }^{*}, \mathrm{p}<0.02\right)$. Error bars represent SEM.

For both normal and affected dogs, PLR latency decreased as stimulus intensity increased (Figure 3-7). Average latency in normal dogs ranged from 320 milliseconds with a stimulus of $8.5 \mathrm{log}$ photons $/ \mathrm{cm}^{2} / \mathrm{s}$ to 185 milliseconds with a stimulus of $15 \log$ photons $/ \mathrm{cm}^{2} / \mathrm{s}$. The PLR latency was significantly increased in 10 month old CLN2-affected dogs with stimuli between 8.75 and 11 $\log$ photons $/ \mathrm{cm}^{2} / \mathrm{s}(\mathrm{p}<0.01)$. With a stimulus intensity of $10 \log$ photons $/ \mathrm{cm}^{2} / \mathrm{s}$, 
latency was significantly increased by 8 months of age $(p<0.02)$. PLR latency data from 10 month old CLN2-affected dogs was significantly increased from that of 4 and 6 month old affected dogs with stimulus intensities of 9 and $10 \log$ photons $/ \mathrm{cm}^{2} / \mathrm{s}(\mathrm{p}<0.01)$.

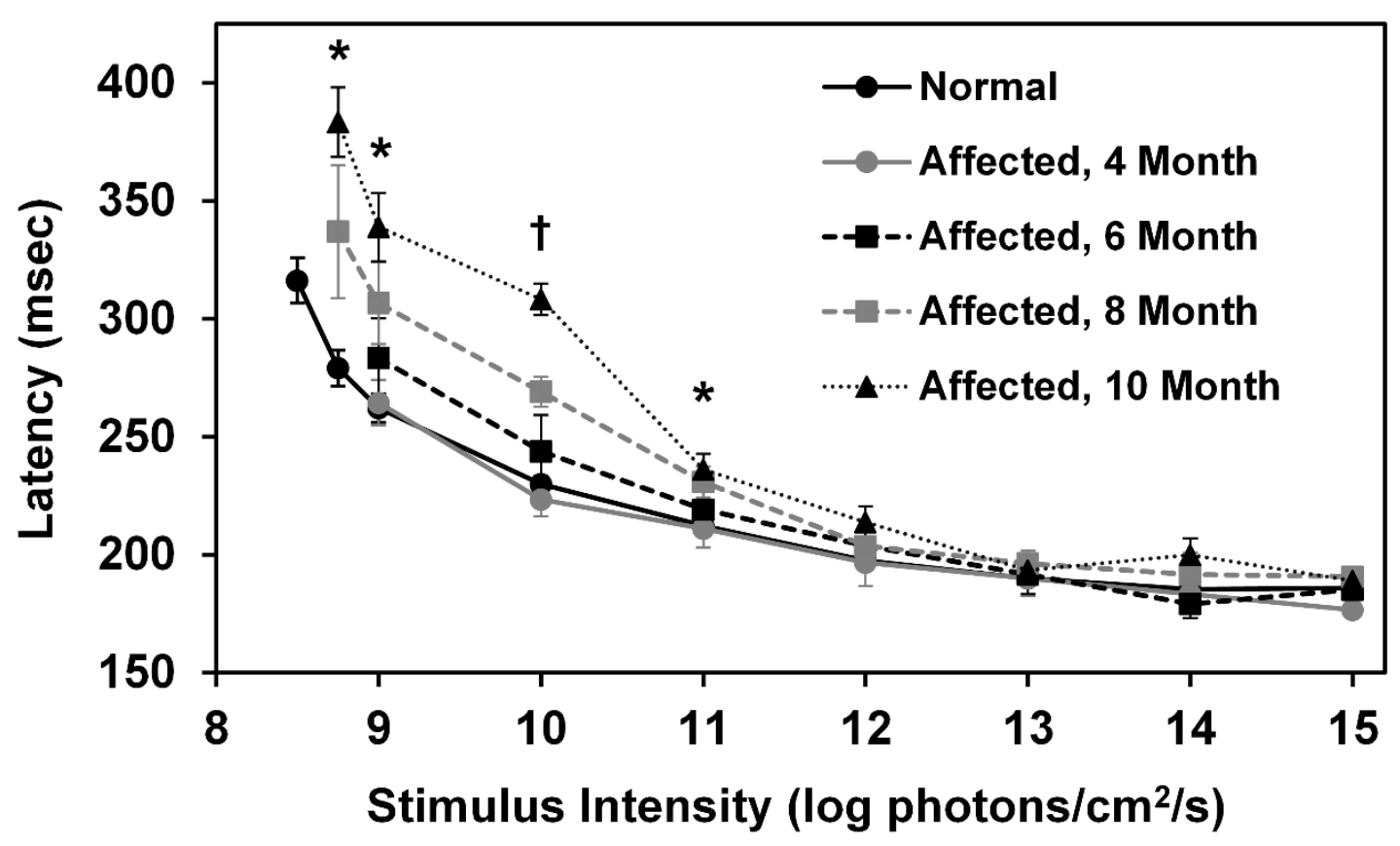

Figure 3-7: PLR latency as a function of stimulus intensity. Latency is significantly longer in CLN2-affected dogs at 10 months of age for stimuli of $8.75,9$, and $11 \log$ photons $/ \mathrm{cm}^{2} / \mathrm{s}\left({ }^{*}, \mathrm{p}<0.01\right)$ and by 8 months for a stimulus of $10 \log$ photons $/ \mathrm{cm}^{2} / \mathrm{s}(\dagger, \mathrm{p}<0.02)$. In addition, the increase in PLR latency was significantly greater in the 10 month old CLN2-affected dogs compared with 4 and 6 month old dogs for stimuli of 9 and $10 \log$ photons $/ \mathrm{cm}^{2} / \mathrm{s}$. Error bars represent SEM.

In both normal and CLN2-affected dogs, average constriction velocity of the PLR increased with increasing stimulus intensity from 8 to $11 \log$ photons $/ \mathrm{cm}^{2} / \mathrm{s}$ at which point it began to decrease slightly with each additional increase of 
stimulus intensity (Figure 3-8). Average constriction velocity was significantly reduced from normal in 10 month old CLN2-affected dogs with stimuli between 8.75 and $11 \log$ photons $/ \mathrm{cm}^{2} / \mathrm{s}(\mathrm{p}<0.001)$. Constriction velocity in 10 month old affected dogs was significantly reduced from that of 4,6 , and 8 month old affected dogs $(p<0.01)$.

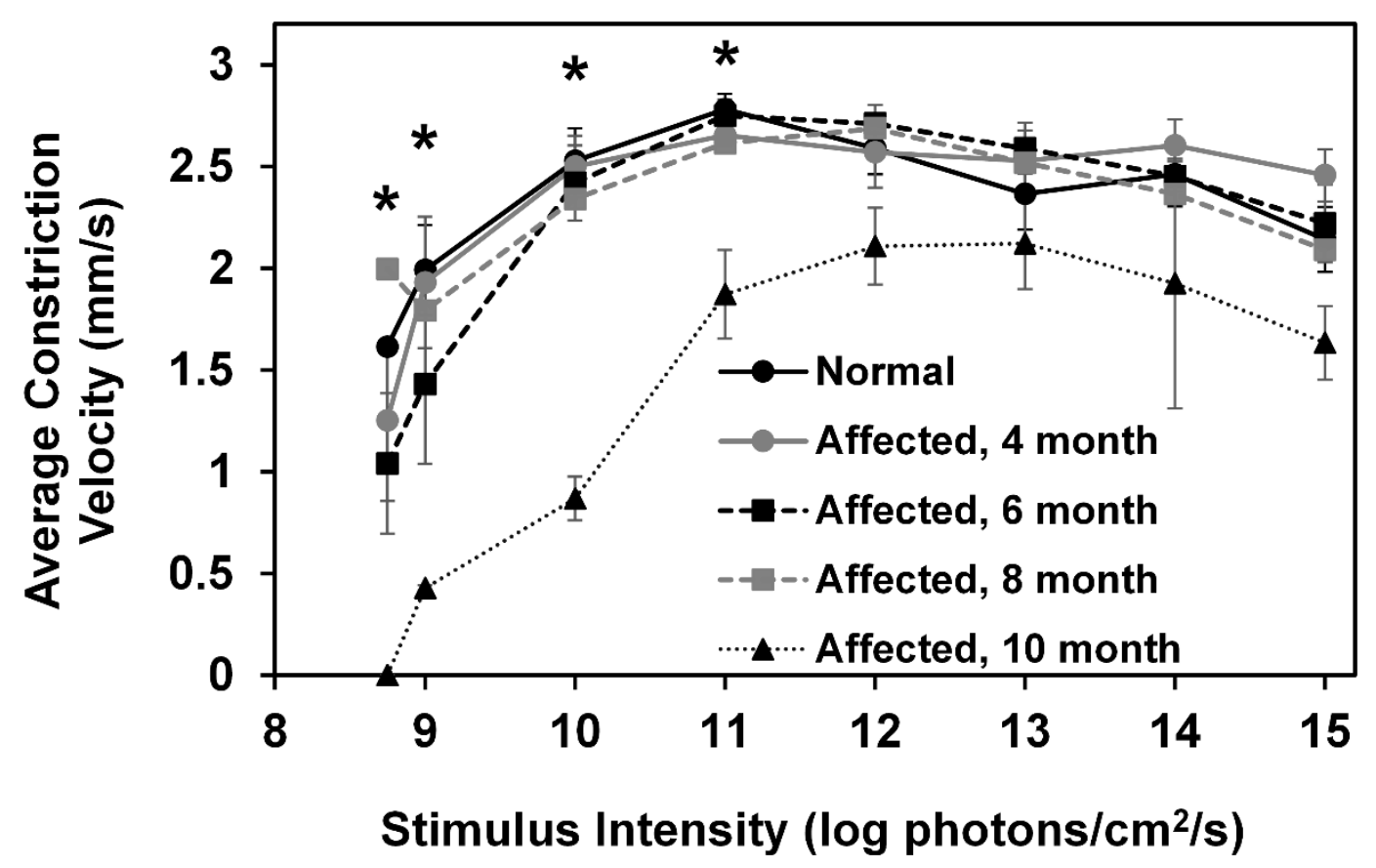

Figure 3-8: Average constriction velocity was significantly reduced in 10 month old CLN2-affected dogs with stimuli between 8.75 and 11 log photons $/ \mathrm{cm}^{2} / \mathrm{s}$ $\left({ }^{*}, p<0.001\right)$. Error bars represent SEM.

In both normal and CLN2-affected dogs, average PLR redilation velocity increased with increasing stimulus intensity from 8 to $11 \log$ photons $/ \mathrm{cm}^{2} / \mathrm{s}$ at which point it began to decrease slightly with each additional increase of stimulus intensity (Figure 3-9). Average redilation velocities of 10 month old affected dogs 
were significantly reduced from normal with stimuli between 8.75 and 11 log photons $/ \mathrm{cm}^{2} / \mathrm{s}(\mathrm{p}<0.005)$. Redilation velocity in 10 month old affected dogs was also significantly reduced from that of 4,6 , and 8 month old affected dogs for a stimulus of $10 \log$ photons $/ \mathrm{cm}^{2} / \mathrm{s}(\mathrm{p}<0.05)$.

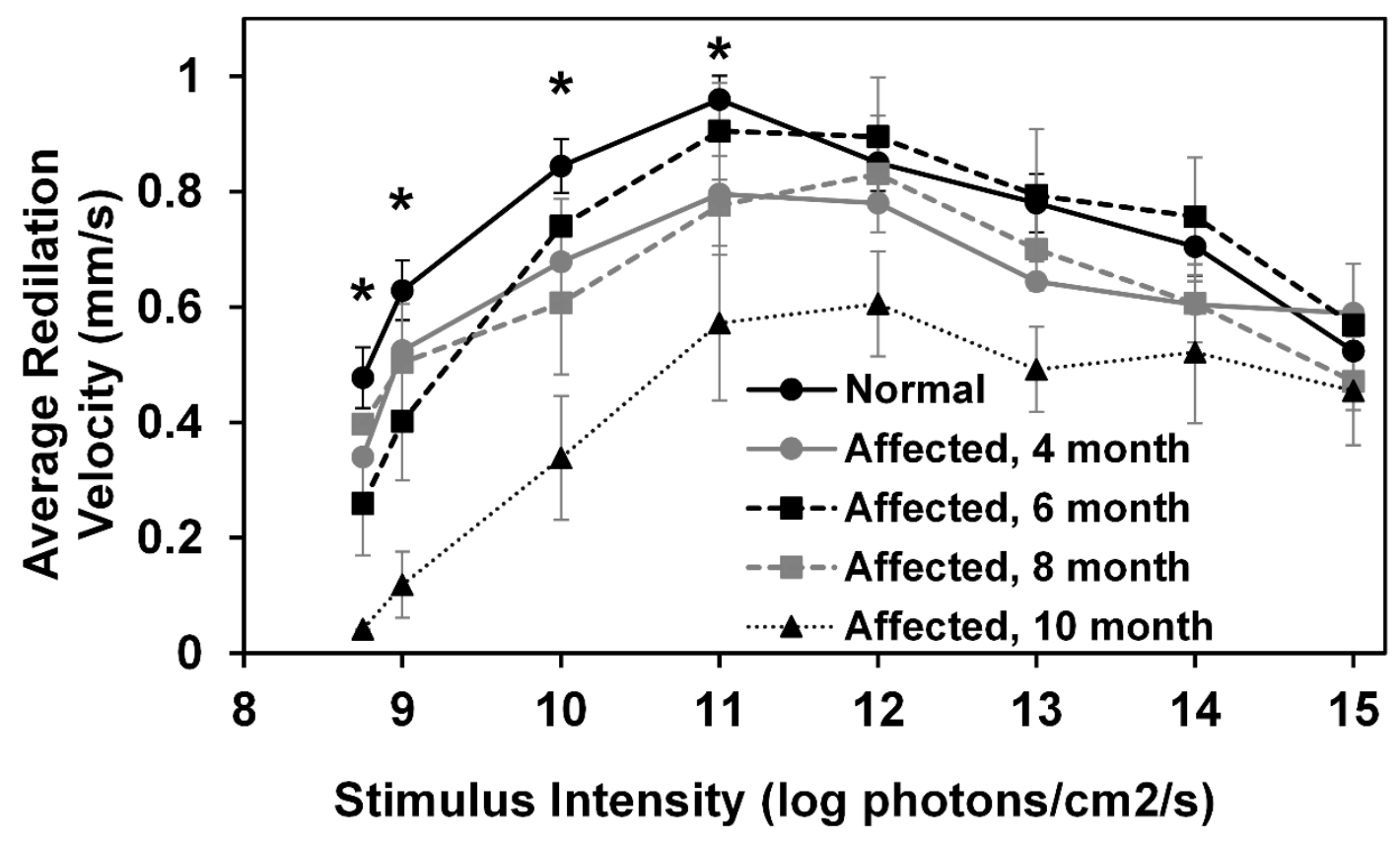

Figure 3-9: Average redilation velocity as a function of stimulus intensity. Redilation velocity is significantly reduced in 10 month old CLN2-affected dogs with stimuli between 8.75 and $11 \log$ photons $/ \mathrm{cm}^{2} / \mathrm{s}\left({ }^{*}, p<0.005\right)$. Error bars represent SEM.

Analysis of sustained pupil size after a bright stimulus (15 log photons $/ \mathrm{cm}^{2} / \mathrm{s}$ ) revealed a significant delay $(p<0.025)$ in secondary redilation of the pupil to baseline size in CLN2-affected dogs at 8 and 10 months of age (Figure 3-10). The rate of secondary redilation was significantly reduced in older affected dogs, 
indicating that post-illumination pupil constriction persisted longer after light offset in these dogs than in normal dogs (Figure 3-10).
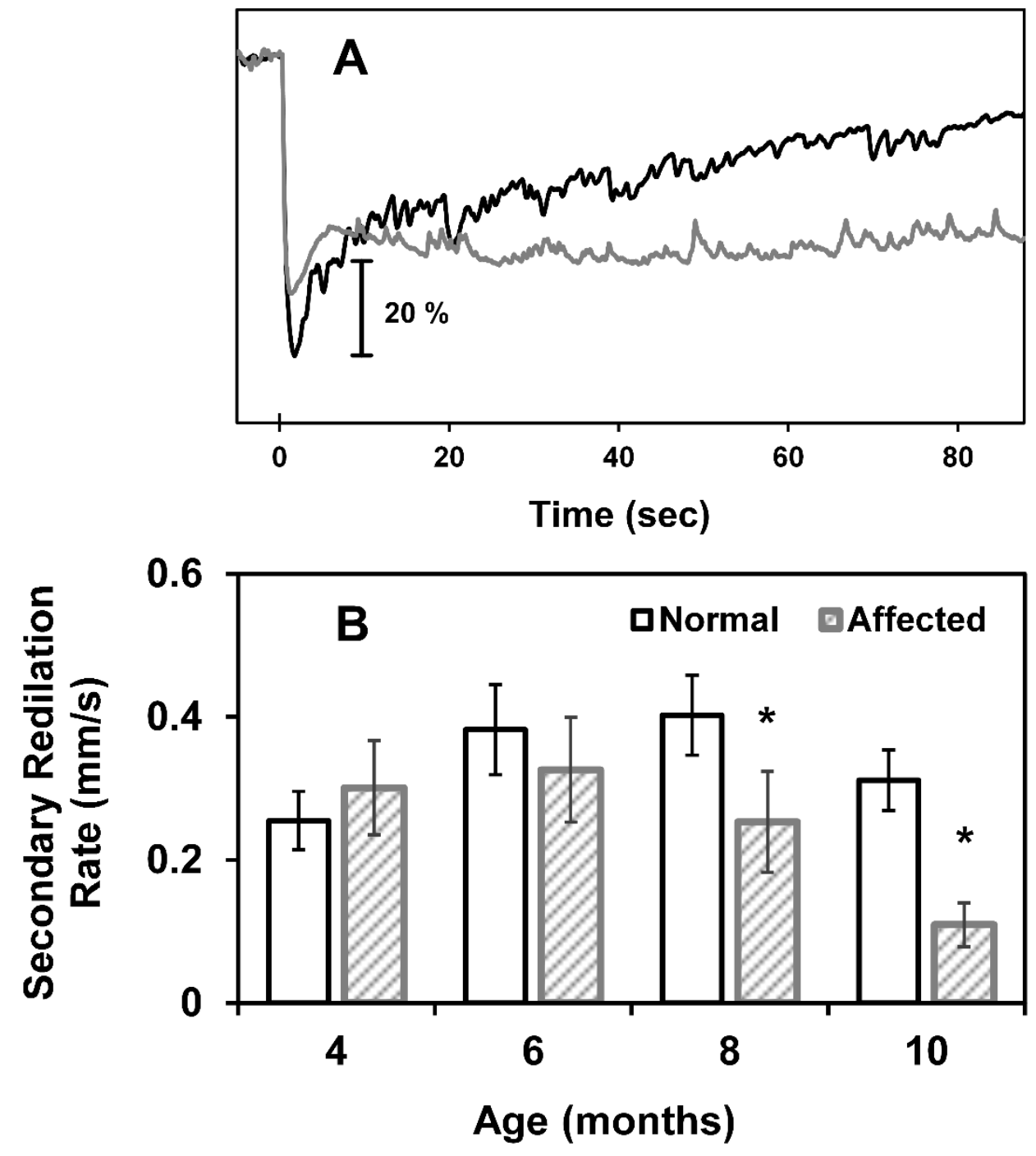

Figure 3-10: The secondary redilation phase is delayed in CLN2-affected dogs at 8 and 10 months of age. (A) Sample PLR traces from 10 month old dogs shown as percent of baseline pupil area. The normal dog pupil gradually redilates to its baseline size while the pupil from the affected dog maintains its constricted size for 85 seconds after light offset. (B) The rate of secondary redilation was decreased in older, affected dogs $\left({ }^{*}, p<0.025\right)$ indicating that constriction persists longer after light offset. 


\subsubsection{Fluorescence Microscopy}

Fluorescence microscopic examination of unstained sections of pretectal neurons and parasympathetic neurons of the oculomotor nucleus revealed a substantial accumulation of autofluorescent inclusions in both brain areas (Figure 3-11). This autofluorescent material was not present in the corresponding neurons from normal dogs.
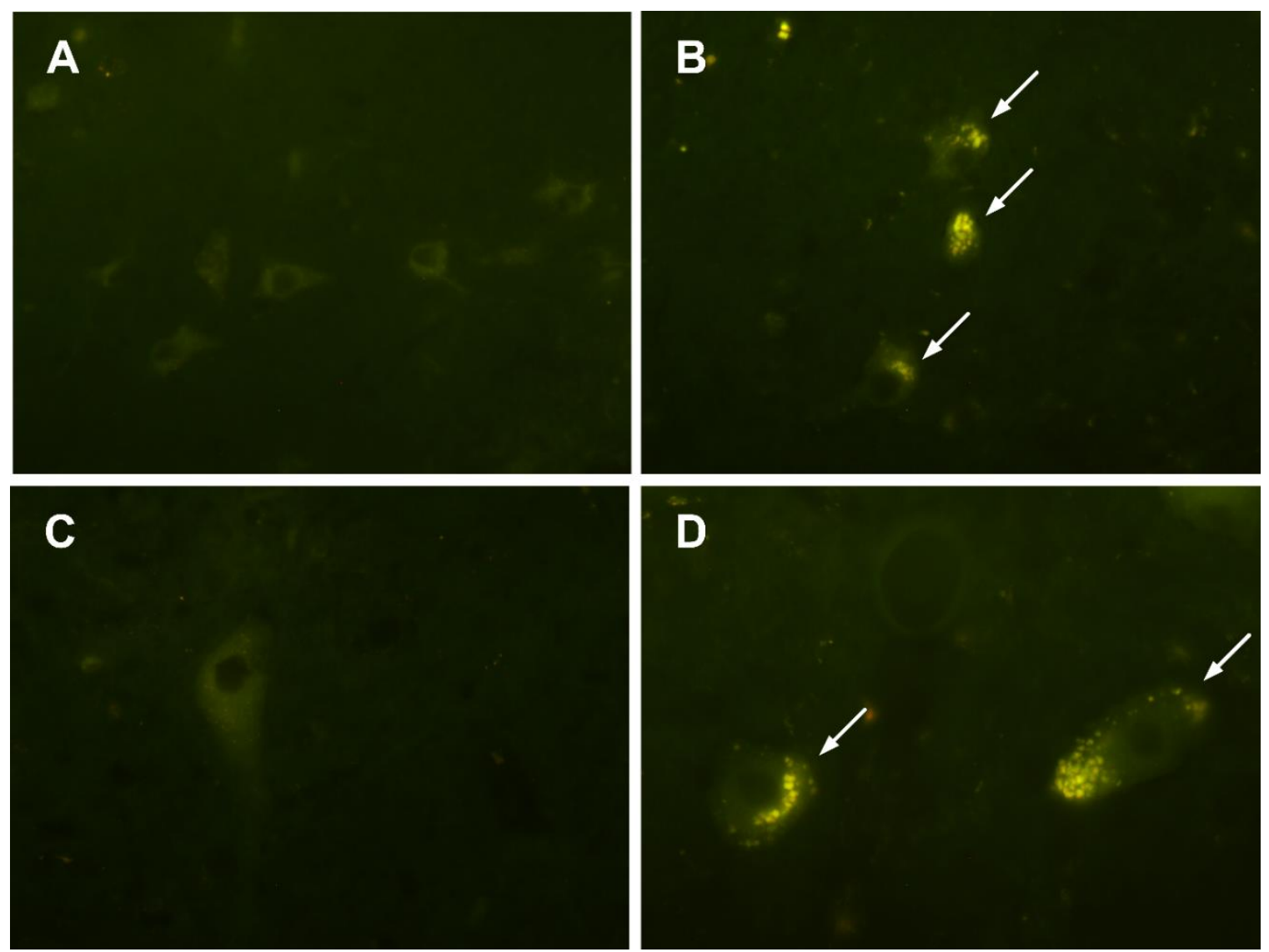

Figure 3-11: Fluorescence micrographs of unstained cryostat sections of pretectal neurons $(A, B)$ and the parasympathetic neurons of the oculomotor nucleus $(C, D)$ from 10.5 month old normal $(A, C)$ and CLN2-affected $(B, D)$ Dachshunds. Yellow-emitting autofluorescent storage material was present in both brain regions of the CLN2-affected dogs. 


\subsection{Discussion}

In this study we have documented clear CLN2 disease-related alterations in both the ERG, which directly measures retinal function, and the PLR, which involves central processing of signals that originated in the retina. Characterization of these deficits in the canine model will be very useful in studies to assess the efficacy of therapeutic interventions for CLN2 that are currently underway.

CLN2 results from the absence of or deficiency in TPP1, a soluble lysosomal enzyme that can be taken up by cells after binding to cell-surface receptors and then transported to lysosomes via the endosomal system (Kyttälä et al. 2006). Therefore, like similar lysosomal storage disorders, CLN2 may be treatable with enzyme replacement therapy (ERT) (Vuillemenot et al. 2011). Because the clinical signs of CLN2, other than vision loss, result almost exclusively from degeneration of the central nervous system, effective treatment of this disorder with ERT will require delivery of functional TPP1 to the brain and spinal cord. Proteins such as TPP1 do not enter the brain or spinal cord parenchyma from the bloodstream, so to be effective therapeutically, the enzyme will need to be delivered directly to these tissues. In a previous study we have shown that TPP1 infused into the cerebrospinal fluid (CSF) is widely distributed throughout the brain (Vuillemenot et al. 2011). More recently, we have demonstrated that longterm infusion of TPP1 into the CSF slows the progression of neurological signs in the Dachshund model of CLN2 (Katz et al. 2013). Therefore, it appears that CSF 
administration of TPP1 has great promise as a means of treating CLN2. However, it seems unlikely that TPP1 infused into the CSF will prevent retinal degeneration. It is possible that TPP1 administered via the CSF will reach retinal ganglion cells via the optic nerve, which is in contact with the CSF, but uptake of the enzyme by other retinal cells via CSF administration seems unlikely. Since retinal degeneration in the Dachshund CLN2 model is characterized primarily by loss of cells within the inner nuclear layer, CSF infusion of TPP1 is unlikely to prevent vision loss in this disease. Therefore, to prevent retinal degeneration and preserve retinal function, it will probably be necessary to supply TPP1 directly to the retina. On the other hand, specific nuclei in the brain that are involved in the PLR would be expected to take up TPP1 from the CSF, so TPP1 administration via CSF infusion may preserve the central pathways that mediate the PLR. In the context of these therapeutic considerations, therefore, it is important to be able to assess both retinal function and the function of central pathways that are dependent on retinal input.

Changes in the PLR of CLN2-affected dogs were apparent particularly with dim stimuli, including significant deficits in constriction amplitude, latency, constriction velocity, and initial redilation velocity which all depend largely on input from the parasympathetic system. Given the strong correlation between constriction amplitude and these other parameters, deficits in all parameters are likely tied to one another and indicate reduced parasympathetic input to the PLR. The stimulus intensities with which deficits were observed are below the 
threshold of melanopsin input indicating that the PLR response with these stimuli would be mainly driven by the rod/cone input (Lucas et al. 2003). Lall et. al. (2010) found that cone input dominates the PLR with stimuli between 8 and 11 $\log$ photons $/ \mathrm{cm}^{2} / \mathrm{s}$, while intrinsic melanopsin input dominates the PLR with stimuli greater than $11 \log$ photons $/ \mathrm{cm}^{2} / \mathrm{s}$ (Lall et al. 2010).

CLN2-affected dogs also exhibited delays in the secondary redilation phase of the PLR and a reduction in baseline pupil size. Each of these findings may point to a deficit in sympathetic input to the PLR. Baseline pupil size is mediated by the sympathetic and parasympathetic innervation of the iris. Therefore, in order for the baseline pupil to be more constricted, the ratio of sympathetic input that promotes dilation to the parasympathetic input that promotes constriction must be reduced. While sympathetic innervation to the iris is responsible for the slow secondary redilation to baseline pupil size, melanopsin input from the retina is responsible for the prolonged constriction that is typical after offset of a sufficiently bright stimulus (Kankipati, Girkin, and Gamlin 2010; Markwell, Feigl, and Zele 2010; Dacey et al. 2005b). This sustained pupil constriction was present in both normal and affected dogs, indicating that the melanopsin pathway was still at least partially intact in the affected dogs. However, it is possible that the reduced rate of secondary redilation in older affected dogs may reflect altered sympathetic input to the iris and/or altered input to the ipRGCs from OFF-bipolar and amacrine cells which both provide inhibitory input to the ipRGCs (Belenky et al. 2003; Brown and 
Lucas 2009). Since inner retinal cell function is severely impaired in CLN2, this apparent inability to moderate the prolonged melanopsin signal could result from a decrease or lack of the sustained synaptic inhibitory input that is normally present for ipRGCs (Belenky et al. 2003).

The photoreceptor cell function, as reflected by ERG a-wave amplitudes, appears to be well preserved in CLN2-affected dogs. However, the b-wave responses, mediated by both rods and cones, were severely depressed in the affected dogs, with cone-mediated responses being better preserved. This is consistent with the previous report that CLN2-affected dogs exhibit significant thinning of the inner retinal layers while the photoreceptor layer remains relatively intact (Katz et al. 2008). The decline in signal transmission from the photoreceptor cells reflected by the reduced b-wave responses would certainly be expected to contribute to PLR deficits in response to dim stimuli. This is consistent with the fact that PLR deficits present between 6 and 8 months of age during the period in which ERG deficits progress most rapidly. However, this does not rule out the possibility that the reduced PLR amplitudes resulted, at least in part, from pathological changes in the downstream neurological pathways involved in mediating the PLR. This is particularly true since fluorescence microscopy illustrated those cellular changes characteristic of CLN2 also occur in regions of the midbrain involved in the PLR (Figure 3-11). In addition, a study in mice lacking ON-bipolar cell transmission of the inner retina demonstrated less severe PLR deficits than those seen in mice lacking rod and 
cone input (Thompson et al. 2011). These facts combined with the low threshold of the PLR suggest that it is possible that even with reduced ERG b-wave amplitudes, the signals from the retina could have been sufficient to be associated with normal PLRs had the central neural pathways involved in mediating the PLR been functionally normal.

The value of the VEP in assessing central processing of light-induced signals originating in the retina is less clear as no significant decreases in VEP amplitudes were observed despite substantial declines in ERG b-wave amplitudes. The reasons for this seeming discrepancy are not apparent, but at least in dogs, visually-mediated behavior can be maintained in the face of significant declines in ERG amplitudes (Acland et al. 2001; Narfstrom et al. 2003a; Narfstrom et al. 2003b). This suggests that even reduced input from the retina may be sufficient to maintain the types of responses in the visual cortex that the VEP reflects.

The characterization of disease-related alterations in light induced responses contributes to our understanding of the pathology underlying CLN2. Preventing development of the deficits in the PLR and ERG responses can be used to objectively assess the efficacy of therapeutic interventions for CLN2 that are currently being evaluated. An ideal therapy would prevent the declines in both the PLR and the ERG responses by preventing both retina and brain degeneration. 


\section{CHAPTER 4}

\section{ENZYME REPLACEMENT THERAPY DELAYS CLN2- RELATED PLR DEFICITS}

\subsection{Introduction}

The TPP1 protein (tripeptidyl peptidase-1) is a soluble lysosomal enzyme that plays an important role in protein turnover. Like other soluble lysosomal enzymes, TPP1 is not only localized within lysosomes, but is also secreted from cells and taken back up via cell surface receptors that recognize the mannose 6phosphate adducts which are common to enzymes that normally function within lysosomes (Figure 4-1). After uptake from the cell surface, TPP1 is incorporated in active form into lysosomes (Kyttälä et al. 2006; Sohar, I., Sleat, D. E., Jadot, M. and Lobel 1999). Therefore, in theory CLN2 should be amenable to TPP1 enzyme replacement therapy. TPP1 is synthesized on the rough endoplasmic reticulum as an inactive pro-enzyme. Upon acidification in the lysosome, the protein is activated by cleavage of a peptide fragment from its amino terminal end. Because large molecules such as TPP1 cannot cross the blood-brain barrier, delivery of the enzyme to the brain has been achieved through administration of pro-TPP1 by infusion into the cerebrospinal fluid (CSF) (Chang et al. 2008; Vuillemenot et al. 2011). In the Dachshund CLN2 disease model, we have previously shown that this route of TPP1 administration results in widespread distribution of the active enzyme in many structures of the brain and 
in reduction in the accumulation of neuronal lysosomal storage material that is characteristic of this disease (Vuillemenot et al. 2011; M. Katz et al. 2013).

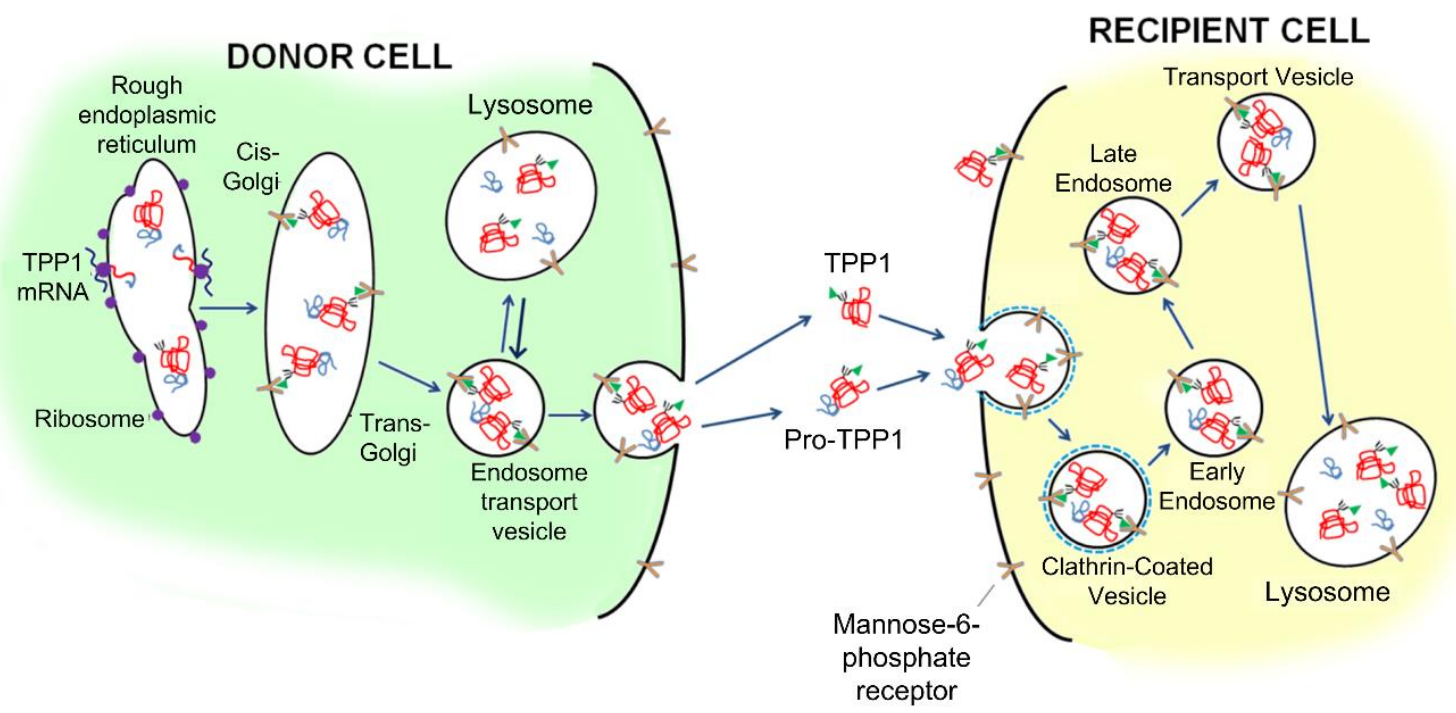

Figure 4-1: Cellular transport of TPP1. TPP1 can be synthesized and transported to the lysosome for use within a single cell, or it can be excreted and taken up by a neighboring cell. With ERT, this results in the uptake and processing of infused TPP1 through normal cellular mechanisms.

CLN2-affected dogs exhibit significant deficits in PLR latency, constriction velocity, constriction amplitude, and redilation velocity, particularly with dim light stimuli. We hypothesize that these PLR deficits result in part from diseaserelated degenerative changes in the CNS nuclei involved in mediating the PLR and that providing TPP1 to the CNS will ameliorate development of these deficits. Studies were therefore undertaken to determine whether chronic administration of recombinant human pro-TPP1 into the CSF could ameliorate PLR deficits associated with CLN2. 


\subsection{Methods}

\subsubsection{Animals}

Studies employed purpose-bred long-haired miniature Dachshunds between 2 and 15 months of age. Dogs were either normal (TPP1+/+; $n=16)$ or CLN2affected (TPP1-/ ; $\mathrm{n}=17)$ as determined by genotyping at the TPP1 locus. All studies were performed in compliance with the ARVO Statement for the Use of Animals in Ophthalmic and Vision Research and were approved by the University of Missouri Animal Care and Use Committee.

\subsubsection{Delivery of Replacement Enzyme}

Recombinant human pro-TPP1 (rhTPP1) was synthesized and purified as described previously (Vuillemenot et al. 2013; Vuillemenot et al. 2011) rhTPP1 was formulated in artificial CSF to concentrations of either 3.33 $\mathrm{mg} / \mathrm{mL}$ or $13.33 \mathrm{mg} / \mathrm{mL}$. Artificial CSF (vehicle) was administered alone to control dogs.

At approximately 2 months of age two catheters were implanted in each dog. One catheter terminated in a lateral ventricle of the brain (ICV catheter) and the other in subarachnoid space at L5 (ITL catheter). The catheters were connected to subcutaneous titanium access ports anchored in the fascia of the muscle and subcutaneous tissues of the cervical (ICV) and lumbar (ITL) regions. Detailed descriptions of the catheter and port implantation procedures are provided elsewhere (Vuillemenot et al. 2013). 
Starting 2 weeks after the catheter implantation surgeries, the test substances were administered via infusion through the ICV catheters once every other week. Three dogs were enrolled in each treatment group listed in Table 4-1. Each infusion of vehicle or $4 \mathrm{mg}$ rhTPP1 was performed over a 2 hour period. Infusions of $16 \mathrm{mg}$ rhTPP1 were initially conducted over a 2 hour period, but the duration was increased to 4 hours to mitigate dosing reactions (Vuillemenot et al. 2013). Infusion of $48 \mathrm{mg}$ rhTPP1 was performed over a 4 hour period for all infusions. The 2 and 4 hour infusion rates were 0.6 or $0.3 \mathrm{~mL} /$ hour, respectively.

\section{Table 4-1: Experimental Treatment Groups}

\begin{tabular}{cc}
\hline Genotype & Treatment \\
\hline TPP1+/+ & Vehicle \\
TPP1+/+ & $4 \mathrm{mg} \mathrm{rhTPP1}$ \\
TPP1+/+ & $16 \mathrm{mg}$ rhTPP1 \\
TPP1-/- & Vehicle \\
TPP1-/- & $4 \mathrm{mg}$ rhTPP1 \\
TPP1-/- & $16 \mathrm{mg}$ rhTPP1 \\
TPP1-/- & $48 \mathrm{mg}$ rhTPP1 \\
\hline
\end{tabular}

After 4 months of age, prior to each infusion, the location within the lateral ventricle and patency of the ICV catheter was assessed by computed tomography of the brain after infusion of $0.1 \mathrm{ml}$ of the nonionic contrast agent 
lohexol (reference marker). Over time the ICV catheter eventually either became occluded or the intraventricular portion migrated into the brain parenchyma due to growth of the cranial cavity. When this occurred, the infusion site was switched from the ICV to the ITL catheter. Eventually, the ITL catheters often became occluded as well. When this occurred, the treatment administration was continued by bolus injection of the agent into the subarachnoid space at the cerebellomedullary cistern over 2 minutes.

\subsubsection{PLR \& ERG Evaluation}

Standard PLR recording sessions were repeated in each dog receiving infusions every 2 months between 6 and 12 months of age. All PLR recordings were performed under general anesthesia. The ERG dog diagnostic protocol, as described in Chapter 2, was repeated monthly in each dog between 3 and 12 months of age. PLR and ERG recordings were also obtained at 15 months of age in two affected dogs treated with TPP1 and three normal dogs.

Results from all genetically normal dogs $(T P P 1+/+)$, including those that were left untreated $(n=7)$, those that received infusions of vehicle $(n=3)$ and those that received infusions of rhTPP1 $(n=6)$, were combined to serve as the normal control group. Results from CLN2-affected dogs that received infusions of vehicle $(n=3)$ were combined with those from untreated, affected dogs $(n=5)$ to serve as the disease control group. Repeated measures 2-way ANOVA was used to compare results from affected dogs treated with infusions of rhTPP1 
enzyme to each of these control groups to determine if enzyme replacement therapy was able to normalize any PLR or ERG deficits related to CLN2 disease progression.

\subsubsection{PLR Parameters}

Baseline pupil diameter was defined as the average pupil diameter, over a 1second period, before light stimulus. PLR constriction amplitude was defined as the difference between baseline pupil diameter and minimum pupil diameter occurring within 2.5 seconds after the stimulus. Latency was determined as the time between stimulus onset and the beginning of pupil constriction. Average constriction velocity was calculated as the constriction amplitude divided by the constriction time, or the time between the beginning of pupil constriction and the minimum pupil diameter. Redilation of the pupil is biphasic with a fast initial redilation and slower secondary redilation. Average redilation velocity was calculated for the initial redilation phase as half the constriction amplitude divided by the time required for the pupil to redilate from its minimum diameter to half the baseline pupil size. For the brightest flash (15 log photons $\left./ \mathrm{cm}^{2} / \mathrm{s}\right)$, the rate of secondary redilation was calculated for the period from 15 to 85 seconds after light offset. 


\subsubsection{Tissue Analysis}

Analyses were performed to determine whether the pretectal nucleus and parasympathetic region of the oculomotor nucleus in rhTPP1 treated dogs exhibit the accumulation of autofluorescent storage material that is characteristic of the NCLs or if treatment was able to reduce the amount of storage material. These regions were also analyzed for presence of the rhTPP1 protein to determine if the infused enzyme was distributed to these tissues.

For fluorescence microscopy, regions of the brain were collected shortly after euthanasia and incubated in $0.1 \%$ glutaraldehyde, $3.5 \%$ paraformaldehyde, 0.13 M sodium cacodylate, and $0.1 \mathrm{mM} \mathrm{CaCl} 2(\mathrm{pH} 7.4)$ with gentle agitation for approximately 24 hours. The samples were then incubated in $0.17 \mathrm{M}$ sodium cacodylate, $\mathrm{pH} 7.4$ with gentle agitation for at least 1 hour, embedded (TissueTek; Sakura Finetek, Torrance, CA), and frozen on dry ice. Five-micrometerthick sections were cut from the Tissue-Tek-embedded samples and mounted on glass slides (Super Frost; Fisher Scientific, Pittsburgh, PA) in 0.17 M sodium cacodylate. The sections were examined with a microscope (Axiophot; Carl Zeiss Meditec, Inc., Dublin, CA) equipped with epi-illumination from a high-pressure, 50-W mercury vapor lamp, a 40X objective lens (Plan-Neofluar) with a 1.3 numerical aperture, a 395- to 440-nm band-pass exciter filter, a chromatic beam splitter (FT 460), and an barrier filter (LP 515; all from Carl Zeiss Meditec, Inc.). Photography was performed with an Olympus DP72 digital camera. 
For rhTPP1 analysis, tissue samples were collected shortly after euthanasia and were frozen immediately on dry ice. The frozen samples were stored at $-80^{\circ} \mathrm{C}$ until analyzed for rhTPP1 concentrations. The catalytically active form of rhTPP1 was measured in tissue homogenates using quantitative colorimetric enzyme linked immuno-absorbent assays (ELISAs). These assays are specific for the recombinant human form of TPP1 and do not detect the endogenous canine TPP1 present in genetically normal animals. The tissue ELISA has a limit of detection of $25 \mathrm{pg} / \mathrm{mL}$ and a range of $1.0-64 \mathrm{ng} / \mathrm{mL}$ in neat tissue homogenate. Tissue ELISA results were normalized to grams total protein in each homogenate, as determined with a BCA protein assay kit (Pierce, Rockford, IL).

\subsection{Results}

\subsubsection{PLR}

CLN2-affected dogs that are left untreated exhibit significantly reduced PLR constriction amplitudes by 8 months of age with stimuli between 9 and $12 \log$ photons $/ \mathrm{cm}^{2} / \mathrm{s}$ (Figure 4-2, Figure 4-3). These deficits are even more pronounced by 10 months of age (Figure 4-2, Figure 4-4).

With all CLN2-affected dogs treated with rhTPP1 grouped together, these dogs have PLR constriction amplitudes that apparently begin to decrease from normal at 8 months of age, though the deficits are not as severe as those of 8 month old untreated, affected dogs (Figure 4-3A). The deficits then appear to progress further by 10 months of age (Figure 4-4A). However, closer 
examination of the data revealed a grouping among the treated dogs that did not depend on treatment dose level. Three of the treated dogs $(2$ dogs treated with 4 mg TPP1 and 1 dog treated with $16 \mathrm{mg}$ TPP1) retained normal PLR constriction amplitude through 10 months of age (Group 1) (Figure 4-2), while the other treated dogs (Group 2) mimicked the deficits in constriction amplitude observed in untreated, affected dogs at both 8 and 10 months of age (Figure 4-3B, Figure 4-4B).

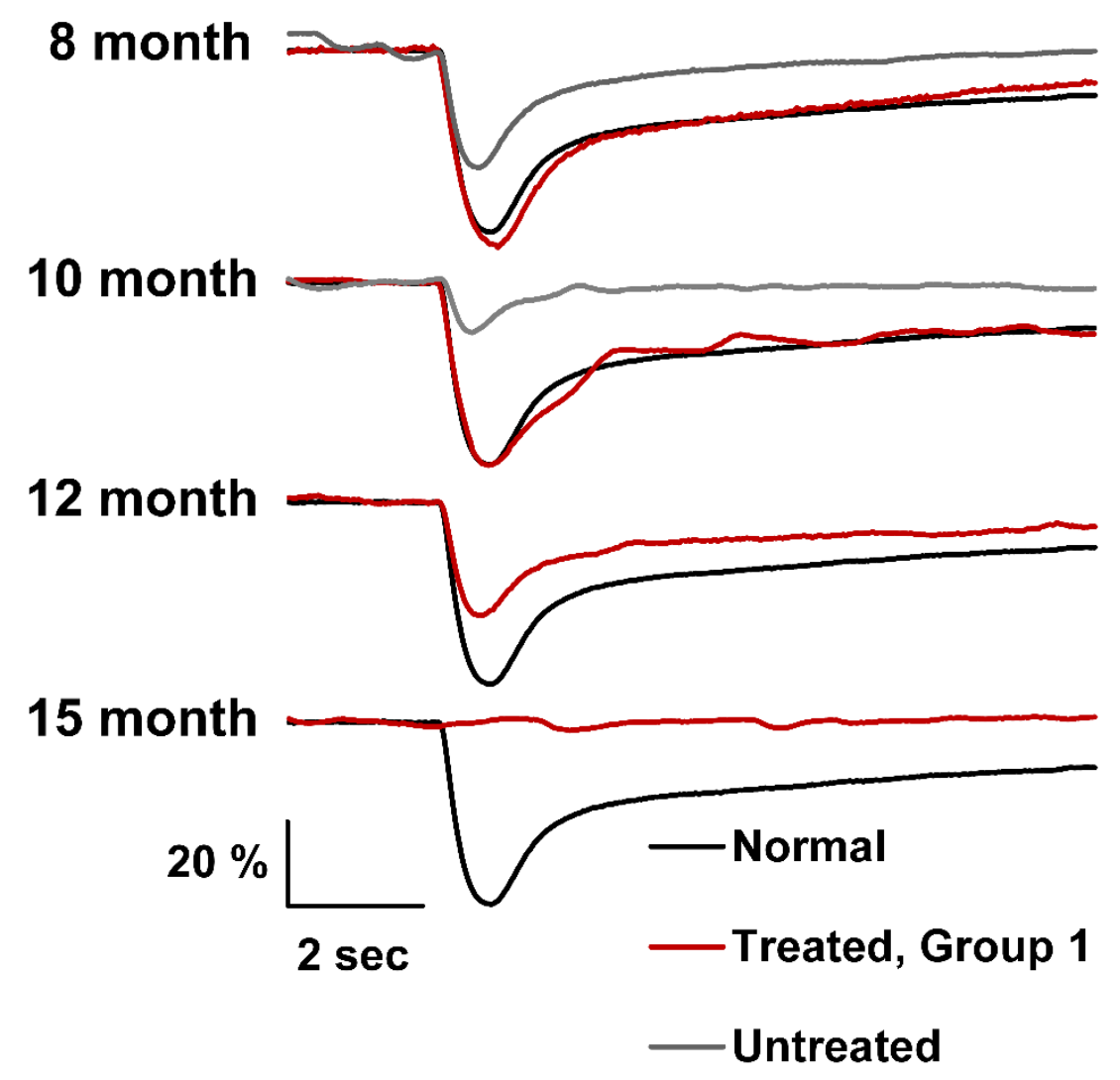

Figure 4-2: Representative PLR traces from a normal dog, untreated CLN2affected dog, and an affected dog that received ERT between 8 and 15 months of age. Pupil responses are shown as percent of baseline pupil area. PLRs are in response to a stimulus of $10 \log$ photons $/ \mathrm{cm}^{2} / \mathrm{s}$. 

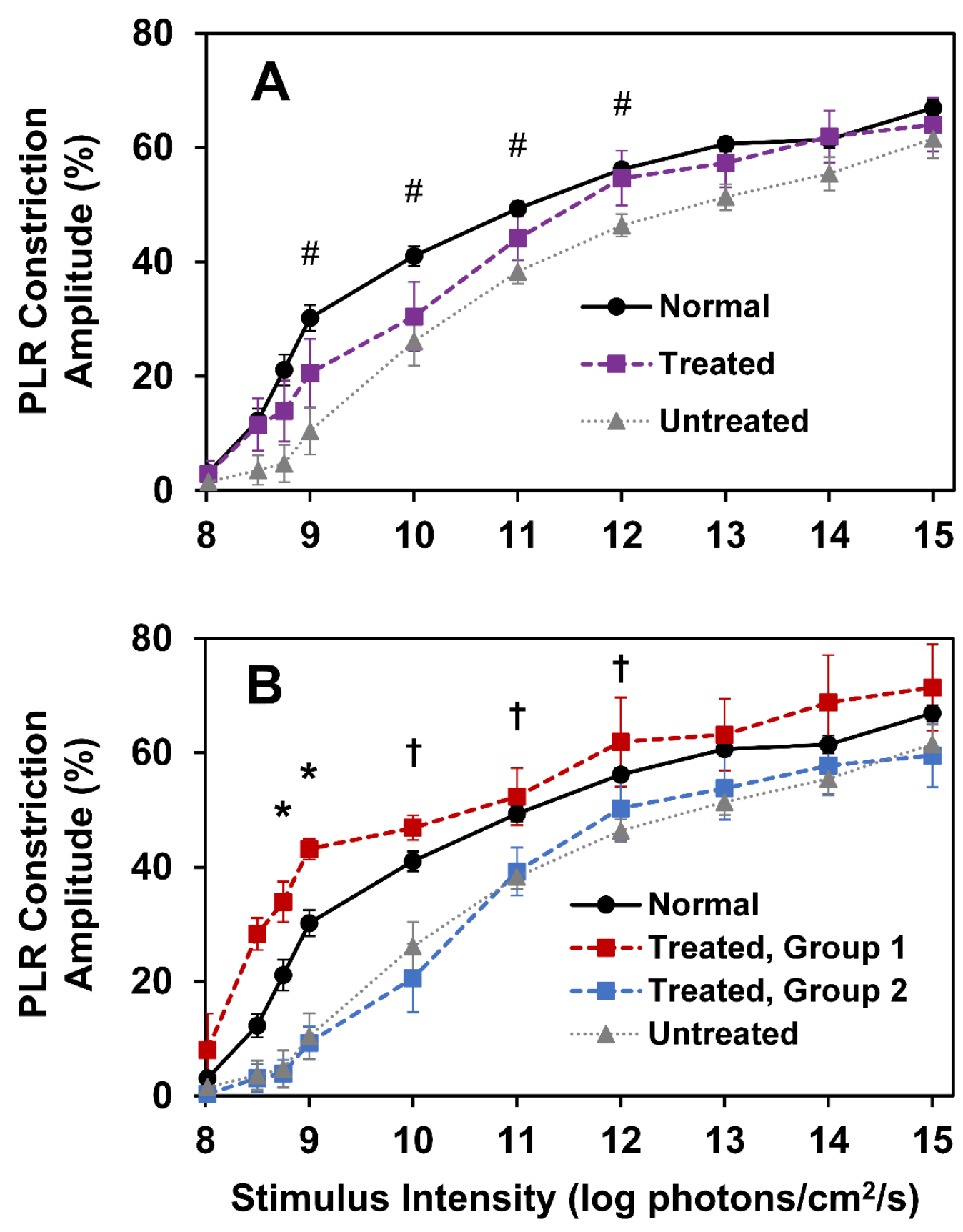

Figure 4-3: PLR constriction amplitudes from 8 month old dogs. (A) Data from all CLN2-affected dogs treated with TPP1 enzyme replacement therapy is combined. While untreated dogs have significant deficits compared to normal (\#, $p<0.02$ ), treated dogs are not significantly different from normal or untreated dogs. (B) Treated data is broken into 2 groups of dogs with Group 1 having normal constriction amplitudes that are significantly different from those of untreated, CLN2-affected dogs $\left({ }^{*}, p<0.001 ; \dagger, p<0.01\right)$. 

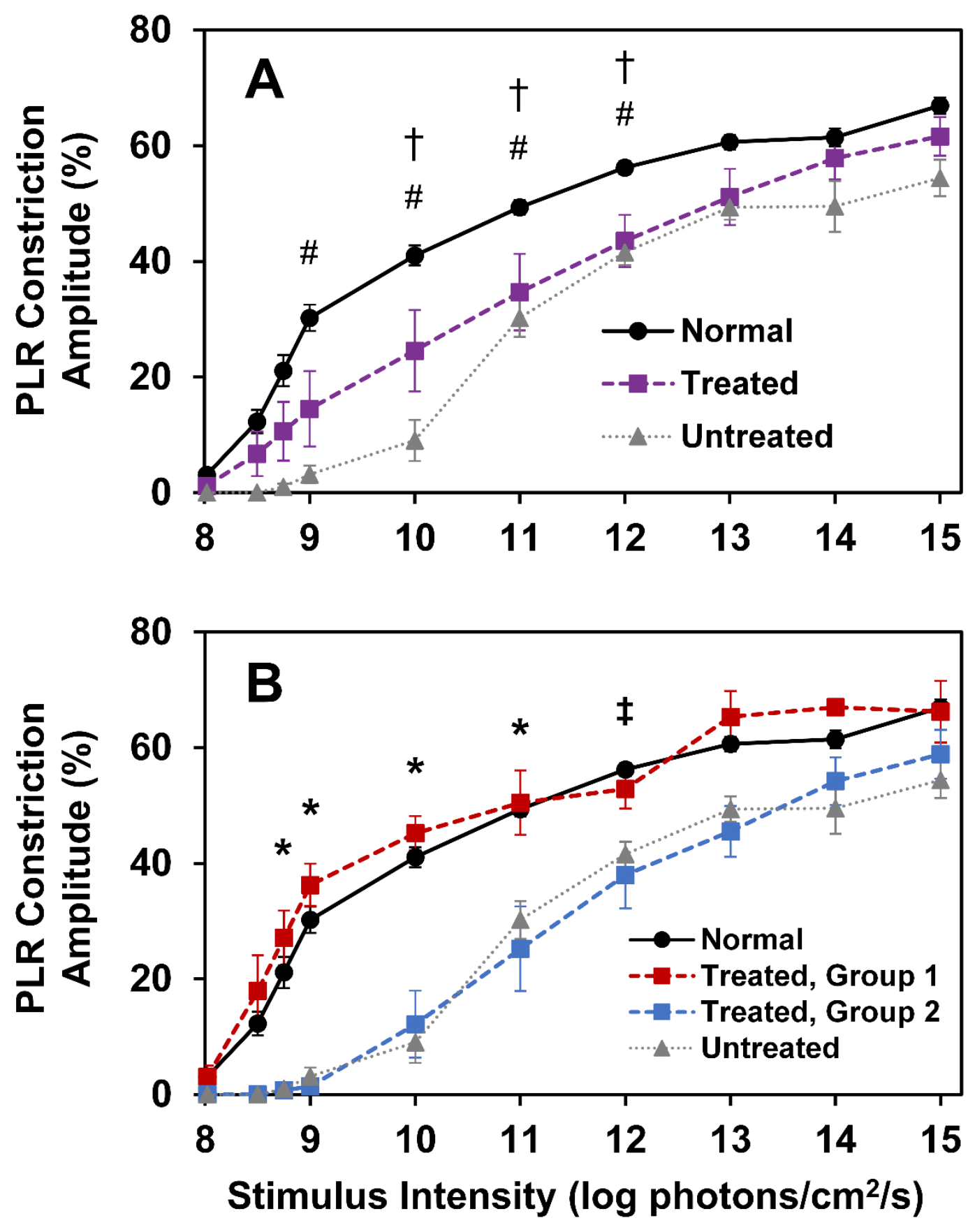

Figure 4-4: PLR constriction amplitudes from 10 month old dogs. (A) Data from all CLN2-affected dogs treated with TPP1 enzyme replacement therapy is combined. Untreated dogs $(\#, p<0.02)$ and treated dogs $(\dagger, p<0.025)$ have significant deficits compared to normal. (B) Treated data is broken into 2 groups of dogs with Group 1 having normal constriction amplitudes that are significantly different from those of untreated, CLN2-affected dogs $\left({ }^{*}, p \leq 0.001 ; \ddagger, p<0.025\right)$. 
By 12 months of age, treated dogs from Group 1 began to show PLR deficits that indicated a waning effect of the treatment (Figure 4-5). However, constriction amplitudes in Group 1 were still significantly increased over those of Group 2 for stimuli of 9 and $10 \log$ photons $/ \mathrm{cm}^{2} / \mathrm{s}(\mathrm{p}<0.05)$.

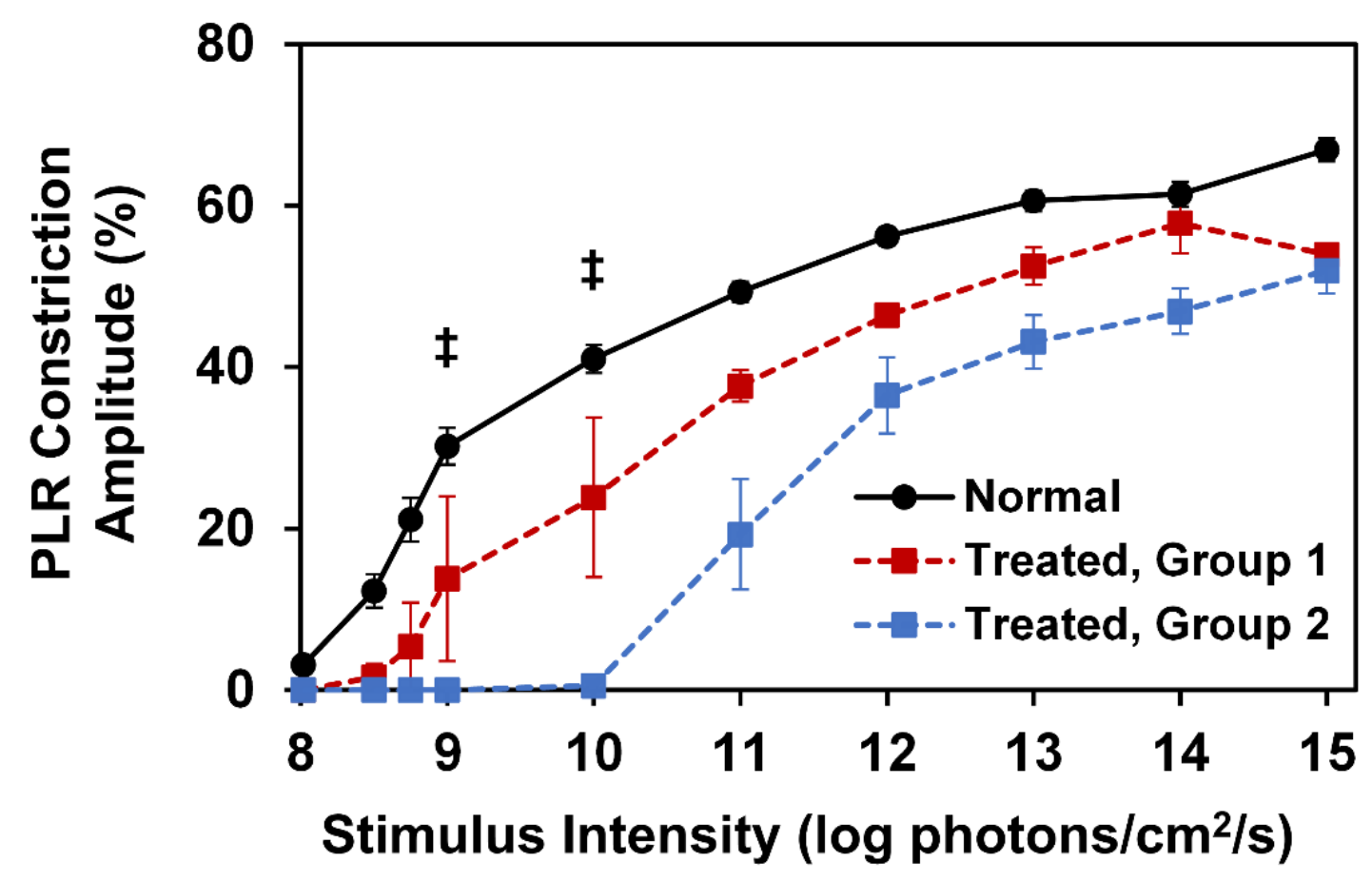

Figure 4-5: PLR constriction amplitudes from 12 month old CLN2-affected dogs. Amplitudes are reduced from normal in both Group 1 and Group 2, though Group 1 amplitudes are still significantly greater $(\ddagger, p<0.05)$ than those of Group 2 for stimuli of 9 and $10 \log$ photons $/ \mathrm{cm}^{2} / \mathrm{s}$.

Deficits in PLR constriction amplitude at 15 months of age (Figure 4-6) were very similar for both dogs treated with rhTPP1 that survived to this age (one dog from each group). However, the change between 12 months and 15 months of age was much more pronounced in the dog from Group 1 (Figure 4-6A). PLR 
threshold at 15 months of age was increased by over 3 log units from the average threshold for this group at 12 months of age. For the 15 month old dog from Group 1, these changes coincided with end-stage disease. However, the 15 month old dog from Group 2 had only begun to show neurologic signs of disease. Both 15 month old dogs had severe deficits in inner retinal function as illustrated by reduction of the ERG b-wave amplitude.

Similar trends to those found with PLR constriction amplitude were observed for PLR latency, constriction velocity, and redilation velocity. The group of CLN2affected dogs that received rhTPP1 and exhibited normal PLR constriction amplitude (Group 1) also had normal values for these parameters at 10 months of age (Figure 4-7). 

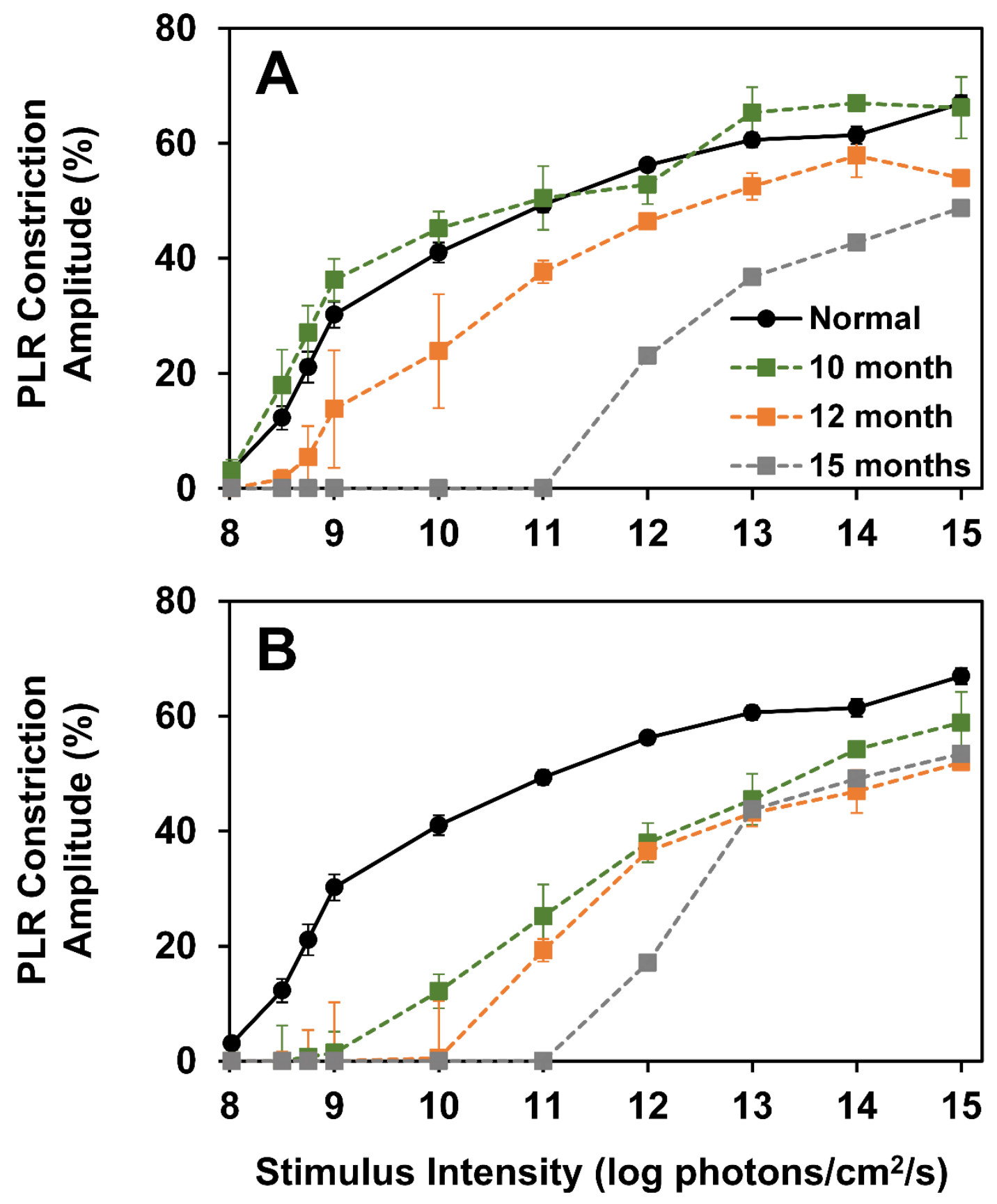

Figure 4-6: PLR constriction amplitude changes with age for rhTPP1 treated dogs from Group 1 (A) and Group 2 (B). Group 1 shows a delay in PLR constriction amplitude deficits, but at 15 months of age, the dogs from each group exhibit very similar results. 

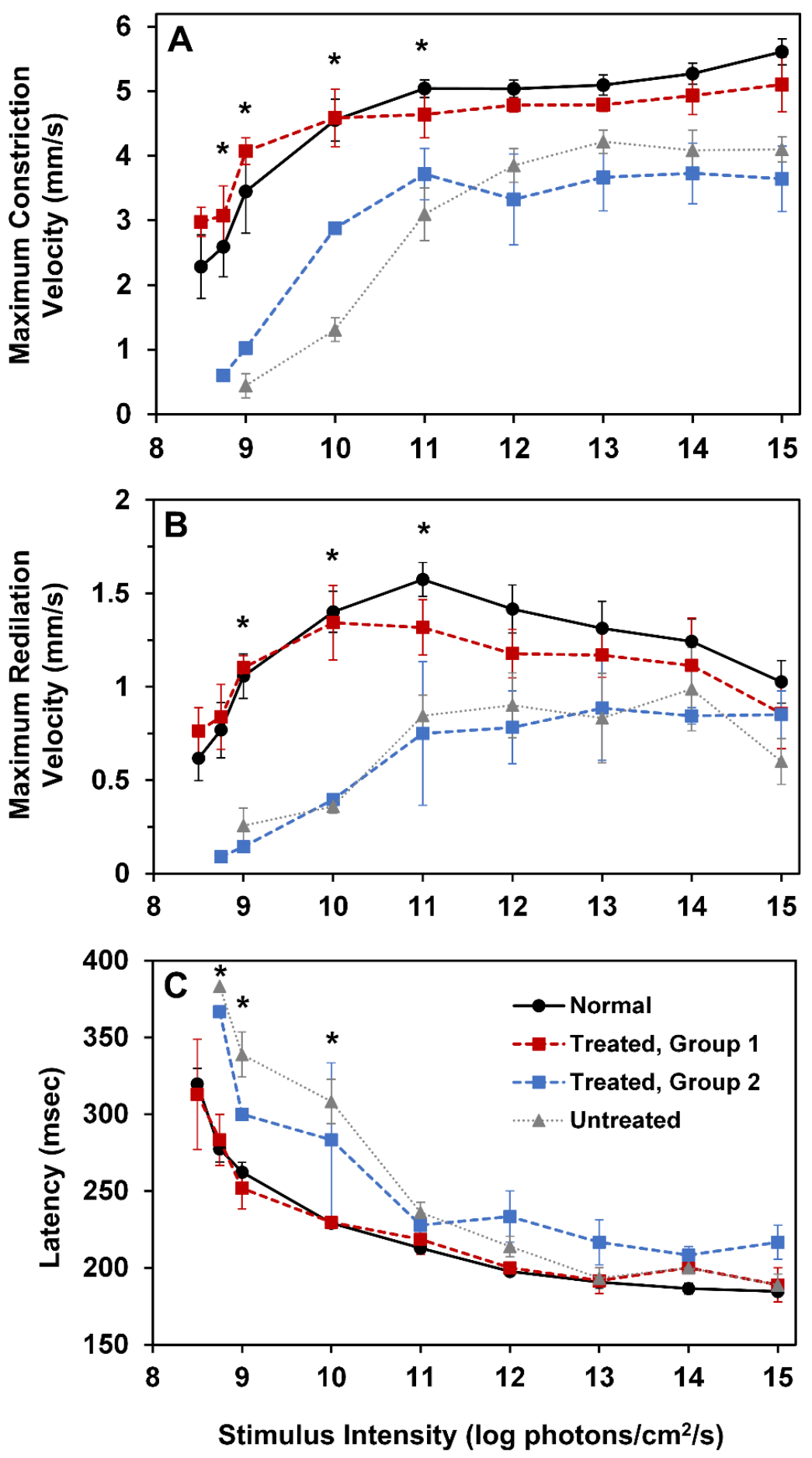

Figure 4-7: PLR parameters in normal and 10 month old treated and untreated CLN2-affected Dachshunds. Maximum constriction velocity (A), maximum redilation velocity $(B)$, and latency $(C)$ are all normalized in Group 1 of the treated dogs and are significantly different than untreated dogs with dim intensity stimuli $\left({ }^{*}, p<0.05\right)$. 
Analysis of sustained pupil size after a bright stimulus (15 log photons $/ \mathrm{cm}^{2} / \mathrm{s}$ ) revealed a significant delay $(p<0.05)$ in secondary redilation of the pupil to baseline size in rhTTP1 treated CLN2-affected dogs (Figure 4-8), similar to deficits found in untreated dogs (Chapter 3 ). There was no difference in this measure between Group 1 and Group 2 treated dogs. The rate of secondary redilation was significantly reduced in treated affected dogs than in genetically normal dogs between 8 and 12 months of age, indicating that post-illumination pupil constriction persisted longer after light offset in these dogs than in normal dogs.

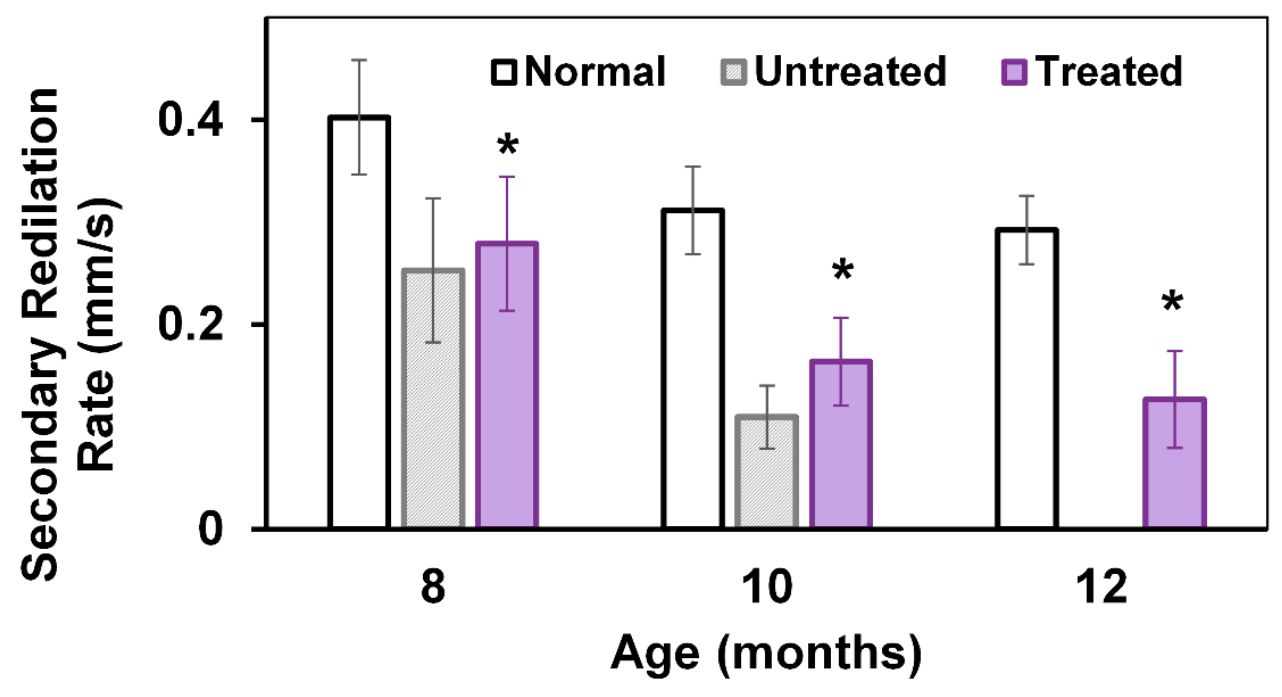

Figure 4-8: The secondary redilation phase of the PLR is delayed in treated CLN2-affected dogs between 8 and 12 months of age. The rate of secondary redilation was significantly decreased $\left({ }^{*}, p<0.05\right)$ indicating that constriction persists longer after light offset in these dogs than in normal dogs. 


\subsubsection{ERG and VEP}

Deficits in both scotopic and photopic ERG b-wave amplitudes of CLN2affected dogs that were treated with rhTPP1 were very similar to those of untreated, affected dogs (Figure 4-9). No improvement was seen in b-wave amplitudes of Group 1 dogs compared with either untreated dogs or treated dogs from Group 2.

The amplitudes of the VEPs elicited under photopic conditions were quite variable in both normal and affected dogs. Dogs treated with rhTPP1 showed no clear difference from those of untreated, affected dogs. 

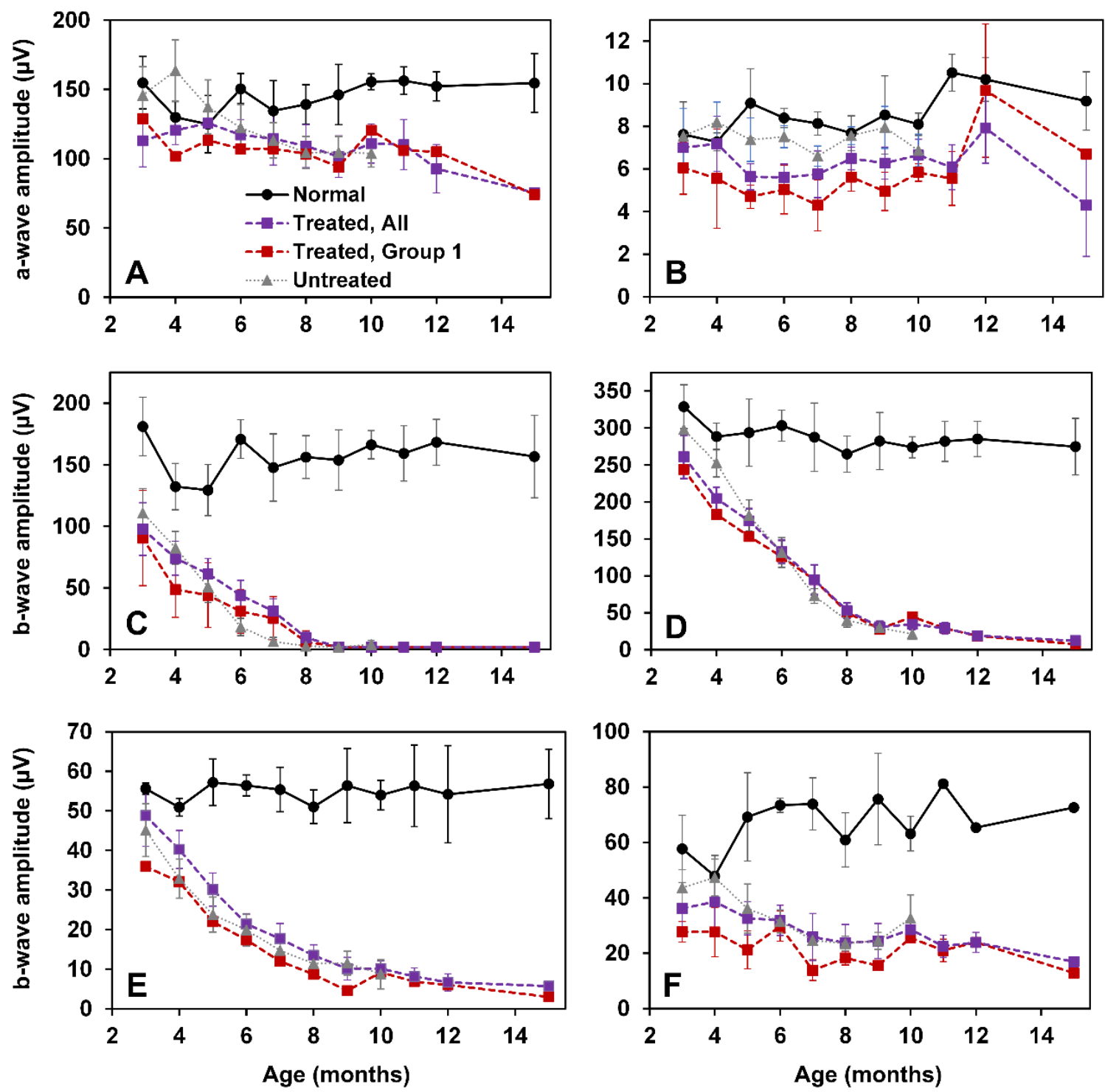

Figure 4-9: $E R G$ a-wave $(A, B)$ and b-wave $(C-F)$ amplitudes as a function of age in normal and CLN2-affected Dachshunds. ERG deficits are similar in CLN2-affected dogs that were untreated and those that received TPP1 ERT. ERG a-wave amplitude from $10 \mathrm{~cd} . \mathrm{s} / \mathrm{m}^{2}$ scotopic (A) and photopic (B) recordings. ERG b-wave amplitude from scotopic rod responses (C), scotopic mixed responses from rods and cones at $10 \mathrm{~cd} . \mathrm{s} / \mathrm{m}^{2}$ (D), photopic cone recordings $(E)$, and photopic $30 \mathrm{~Hz}$ flicker recordings $(F)$. Treated dogs from Group 1 that exhibited normal PLR constriction amplitudes, exhibit no improvement in ERG b-wave amplitude. Error bars represent SEM. 


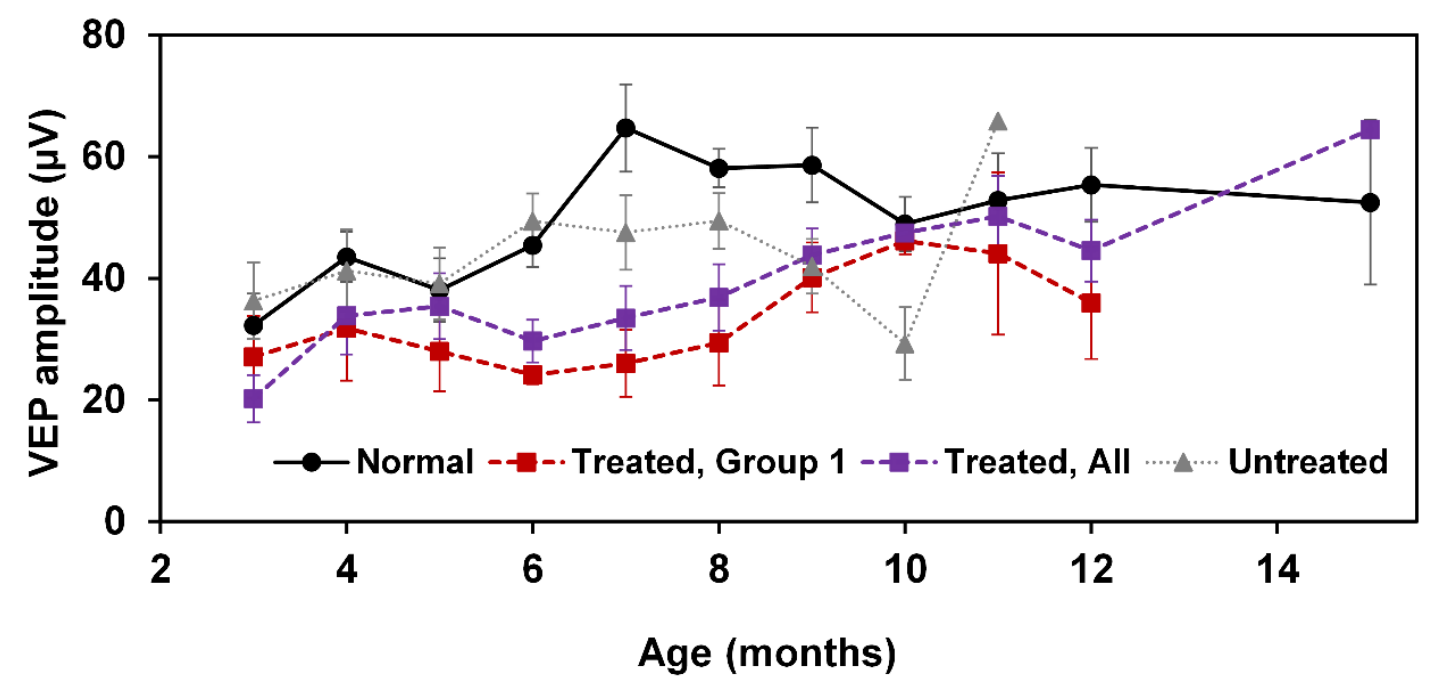

Figure 4-10: VEP amplitudes as a function of age. Results were variable and no differences were apparent amongst the treatment groups.

\subsubsection{Tissue Analysis}

rhTPP1 enzyme was detectable in both the pretectal nuclei and parasympathetic nuclei of the oculomotor nucleus in 4 of 5 treated dogs that were examined (the tissue from 1 dog was not analyzed). Fluorescence microscopic examination of unstained sections of the pretectal nucleus and the nuclei of the parasympathetic division of the oculomotor nucleus of treated CLN2-affected dogs revealed a much lower prevalence of autofluorescent inclusions in neurons in both nuclei when compared with untreated dogs (Figure 4-11). This autofluorescent material was not present in the corresponding brain areas from normal dogs. 

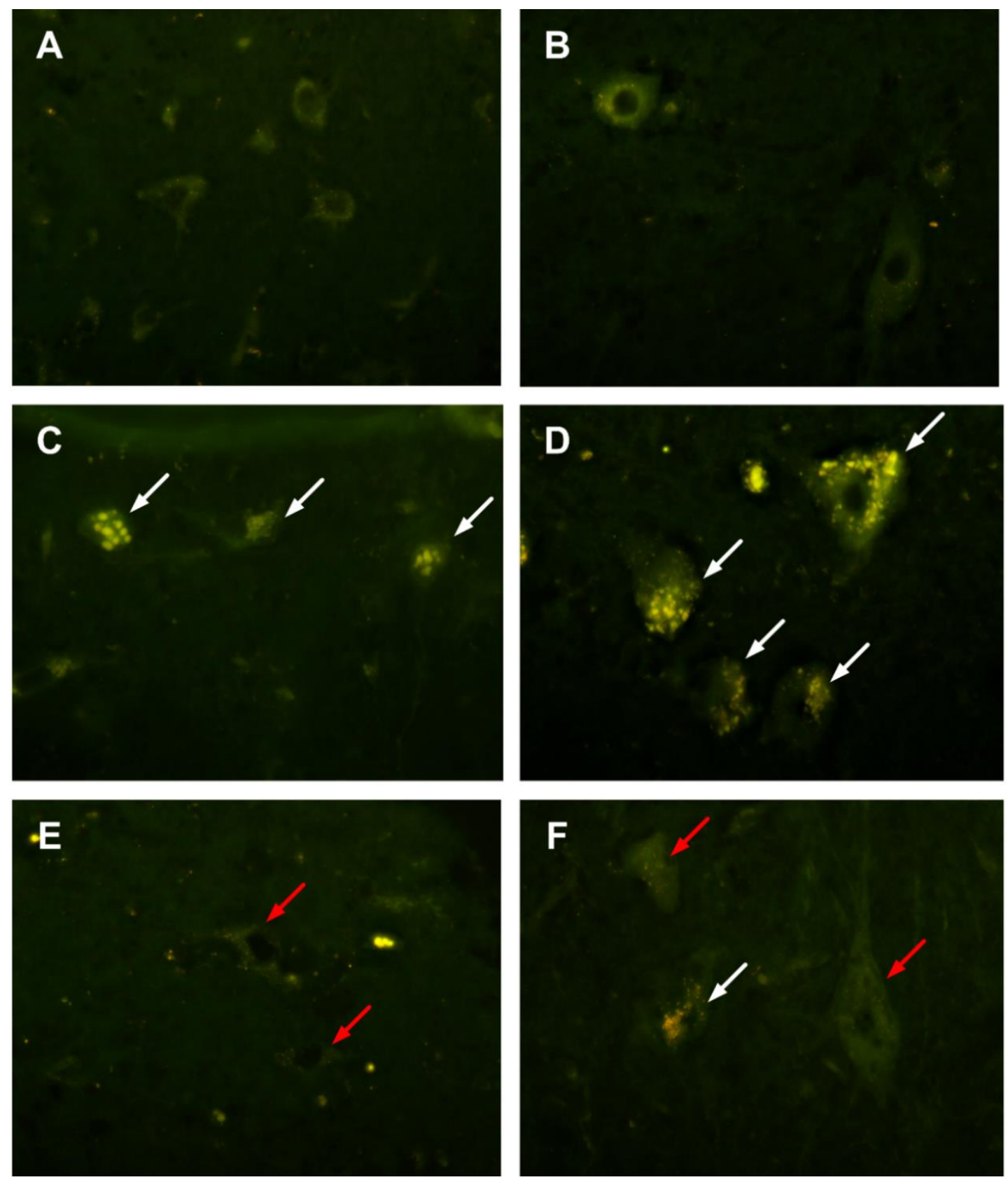

Figure 4-11: Fluorescence micrographs of unstained cryostat sections of the pretectal nucleus (left column; A,C,E) and the parasympathetic division of the oculomotor nucleus (right column; B,D,F) from normal dogs $(A, B)$, untreated CLN2-affected dogs (C,D) and affected dogs treated with rhTPP1 (E, F). Yellowemitting autofluorescent storage material was present in the neurons of both nuclei in the untreated CLN2-affected dogs (white arrows). While some storage material was present in the dogs treated with rhTPP1, it was much less prevalent and unlike the untreated dogs, there are neurons that do not appear to contain this material (red arrows). 


\subsection{Discussion}

Based on the results from this study, it appears that pro-TPP1 delivered directly to the brain via the CSF has the ability to delay PLR deficits associated with CLN2 in a Dachshund model, despite the fact that these treatments did not inhibit the decline in retinal function as assessed with the ERG. The group of CLN2-affected dogs that showed treatment effects had normal PLR constriction amplitude through 10 months of age and didn't begin to display deficits until 12 months of age. This is a great improvement when compared with untreated dogs which began to exhibit deficits by 6 months of age that progressed through endstage disease at 10 months of age.

The PLR depends on the function of both the retina and specific nuclei in the midbrain. All of these tissues exhibit an accumulation of disease-related storage material in CLN2-affected dogs. Based on the CSF circulation, the only retinal neurons that may be expected to obtain rhTPP1 infused into the CSF would be the ganglion cells which may be able to take up the enzyme from the CSF that bathes the optic nerve. It is unlikely that any significant amount of TPP1 was able to reach the cells of the inner nuclear layer of the retina, which undergo severe degeneration in CLN2 (Katz et al. 2008). Consistent with this expectation, rhTPP1 administered via the CSF did not result in any improvement in the ERG b-wave, which reflects activity of retinal neurons with cell bodies located in the retinal inner nuclear layer. Therefore, it seems more likely that the infused enzyme was able to normalize the PLR by preventing degeneration in the 
pretectal and oculomotor nuclei. Consistent with this possibility is the observation that the accumulation of autofluorescent lysosomal storage bodies in neurons in these brain nuclei appeared to be reduced in affected dogs treated with TPP1. In addition, analysis of the pretectal and oculomotor nuclei obtained at necropsy from the treated dogs indicated that the enzyme infused into the CSF did reach these brain nuclei.

Results of this study were variable with only one-third of the subjects exhibiting a treatment effect on the PLR. This may be due to variability in treatment delivery route, as the number of infusions for each route (ICV, ITL, bolus injection) differed for each dog. In addition, the exact distribution pattern of TPP1 via the CSF may have been different for each dog resulting in varying treatment effects. Such variability would be expected based on the known flow dynamics of the CSF. The fact that only some dogs responded to the treatment with preservation of the PLR indicates that this therapeutic effect is possible with CSF administration of TPP1, but that it will be important to identify the variables that determine efficacy.

Both treated and untreated CLN2-affected dogs exhibited delays in the secondary redilation phase of the PLR. Melanopsin input to the PLR is responsible for the prolonged constriction that is typical after offset of a sufficiently bright stimulus (Kankipati, Girkin, and Gamlin 2010; Markwell, Feigl, and Zele 2010; Dacey et al. 2005b). It is possible that the reduced rate of secondary redilation in older affected dogs may reflect altered sympathetic input 
to the iris, and/or altered effect on the ipRGCs from OFF-bipolar and amacrine cells which both provide inhibitory input to the ipRGCs (Belenky et al. 2003; Brown and Lucas 2009). ERT was unable to normalize this phase of the PLR despite the improvement observed in other PLR parameters. Since ERT was most likely unable to attenuate retinal degeneration, this may point to a lack of retinal inhibition as the source of these redilation abnormalities. However, due to the variable distribution of TPP1 via the CSF of treated dogs, it is possible that sympathetic neurons involved in the slow redilation phase of the PLR did not receive therapeutic amounts of TPP1 in these dogs and degeneration of these areas contributed to the delays in secondary redilation.

The timing of changes in the PLR associated with CLN2 do not coincide perfectly with changes in retinal function or with neurologic signs caused by neuronal degeneration. In untreated dogs, mild PLR changes occur early in the disease at 6-8 months of age, which coincides with the most rapidly progressing deficits in the ERG and retinal function. However, no large changes occur in the ERG after 8 months of age while the most extreme PLR deficits occur at 10 months of age and coincide with the appearance of severe neurologic deficits. In the dogs treated with ERT, the timing of retinal function deficits was not altered. These findings suggest that alterations in central neural pathways play a predominant role in the development of PLR deficits in canine CLN2. The onset of neurologic signs was delayed in all ERT treated dogs, though only some also showed a delay in the onset of PLR deficits. There is likely a complicated 
interaction of the deficits in both the retina and other CNS areas involved in modulating the PLR, and further analysis of these tissues is needed to fully explain both the presence of the PLR deficits and the observed treatment effects based on distribution of the infused enzyme. 


\section{CHAPTER 5}

\section{SUMMARY AND FUTURE DIRECTIONS}

We have developed a recording system and protocol capable of reliably quantifying the canine PLR. The system provides great flexibility with respect to stimulus color, duration, and range of intensity allowing for thorough evaluation of many parameters known to affect the PLR.

Using the developed system and methods, we have documented clear CLN2 disease-related alterations in both the ERG, which directly measures retinal function, and the PLR, which involves central processing of signals that originate in the retina. Changes in the PLR of CLN2-affected dogs were apparent particularly with dim stimuli, including significant deficits in constriction amplitude, latency, constriction velocity, and initial and secondary redilation velocity.

Characterization of these deficits in the canine model enabled use of the PLR as a biomarker in assessing efficacy of therapeutic intervention.

ERT with TPP1 delivered directly to the brain via the CSF has demonstrated the ability to delay PLR deficits associated with CLN2 in a Dachshund model. While the results were variable, the group of CLN2-affected dogs that showed treatment effects retained a normal PLR significantly longer than untreated dogs. Based on the known circulation of CSF and the lack of improvement in the ERG, it is most likely that ERT was able to normalize the PLR by preventing degeneration of pretectal and parasympathetic division of the oculomotor nucleus 
and possibly the sympathetic neuronal pathway. However, further analysis of retinal and other CNS tissues are needed to fully explain these treatment effects.

While ERT has proven effective in delaying neurologic signs in the Dachshund model of NCL, it has limitations. This method of enzyme delivery requires repeated administration throughout the life of the patient, which is cumbersome and costly. In addition, the catheters implanted in the brain are invasive and prone to complications over time.

Alternative methods of enzyme delivery are currently under consideration for long term treatment of both the neurologic and visual changes that occur in CLN2. Direct TPP1 gene therapy targeted to the parenchymal cells lining the ventricles of the brain would allow the production of TPP1 and secretion directly into the CSF circulation. In preliminary studies using this approach, we have been able to maintain at least normal sustained CSF TPP1 levels for several months after administration of the gene therapy vector. Based on these preliminary studies, we plan to treat additional dogs and determine whether the gene therapy approach can also preserve the PLR in dogs with CLN2. Another treatment method under development is the use of autologous mesenchymal stem cells which have been genetically modified to produce TPP1. These cells can be injected into the vitreous of the eye or the ventricle of the brain for long term production of TPP1. By treating both central and peripheral tissues affected by CLN2, this method has promise for ameliorating the most prominent symptoms associated with the disease. For the ideal case in which a treatment 
is able to prevent both neurologic and visual deficits, PLR quantification in this dog model will play a large role in demonstrating treatment efficacy. 


\section{APPENDIX A \\ PARTS LIST FOR THE PLR RECORDING SYSTEM}

Super Circuits Inc, Austin, TX: www.supercircuits.com

National Instruments Corp, Austin, TX: www.ni.com

Thorlabs Inc, Newton, NJ: www.thorlabs.com

\begin{tabular}{|l|l|c|l|}
\hline Part Number & Part Name & Quantitiy & Company \\
\hline PC164CEX-2 & Low Light Video Camera & 1 & Super Circuits \\
\hline DC12-1000R & Power Supply & 1 & Super Circuits \\
\hline 776670-35 & LabView Software/Full & 1 & National Instruments \\
\hline 778838-01 & PCl Image card & 1 & National Instruments \\
\hline 183882-02 & IMAQ-BNC, image cable & 1 & National Instruments \\
\hline 780116-01 & DAQ Device, BNC Term & 1 & National Instruments \\
\hline B4C & Cage Cube Platform & 1 & Thorlabs \\
\hline B1C & Cover Plate & 1 & Thorlabs \\
\hline FM03 & Cold Mirror & 1 & Thorlabs \\
\hline B5C & Cage Cube Optic Mount & 1 & Thorlabs \\
\hline C6W & Cage System Cube & 1 & Thorlabs \\
\hline CFH1 & Filter Holder w/Plate & 1 & Thorlabs \\
\hline CFH1-F & Extra Filter Holder & 4 & Thorlabs \\
\hline SM1A9 & Thread Adapter & 1 & Thorlabs \\
\hline SM1T2 & Coupler, External Threads & 2 & Thorlabs \\
\hline SM1D12C & Ring-Activated Diaphragm & 1 & Thorlabs \\
\hline SM1NR05 & Zoom Housing & 1 & Thorlabs \\
\hline SM1A2 & Thread Adapter & 1 & Thorlabs \\
\hline SM2RR & Retaining Ring & 1 & Thorlabs \\
\hline CP02 & Cage Plate & 1 & Thorlabs \\
\hline SM1CP2M & End Cap & 3 & Thorlabs \\
\hline SM1A6 & Thread Adapter & 1 & Thorlabs \\
\hline SM05L03 & Lens Tube & 1 & Thorlabs \\
\hline SM1L10 & Lens Tube & 1 & Thorlabs \\
\hline CM1-BS013 & Beamsplitter & 1 & Thorlabs \\
\hline LA1540-A & Plano-Convex Lens & 1 & Thorlabs \\
\hline AC127-030-B & Near IR Achromat Lens & 1 & Thorlabs \\
\hline NE510B & Absorptive ND Filter, OD: 1 & 1 & Thorlabs \\
\hline NE520B & Absorptive ND Filter, OD: 2 & 1 & Thorlabs \\
\hline NE540B & Absorptive ND Filter, OD: 4 & 1 & Thorlabs \\
\hline MCWHL2 & Cold White Mounted LED & 1 & Thorlabs \\
\hline M470L2 & Blue Mounted LED & 1 & Thorlabs \\
\hline M627L1 & Red Mounted LED & Thorlabs \\
\hline TPS001 & 15 V Power Supply Unit & 1 & Thorlabs \\
\hline LEDD1A & T-Cube LED Driver & 1 & Thorlabs \\
\hline & & & \\
\hline
\end{tabular}




\section{APPENDIX B}

\section{PHOTOSHOP IMAGE ANALYSIS “ACTION” STEPS}

- Create a new action and record the following steps:

- Use the elliptical marquee tool to select the general area of the image that contains the pupil.

- Under the "Select" menu choose "Color Range..."

- Click on the pupil using the eye dropper - choose a color that accurately selects the pupil.

- Under the "Select" menu, choose "Modify" and then "Smooth". Enter the number of pixels by which to smooth the selection -typically between 1 and 5 pixels. This should remove any extraneous pixels that have been selected but are not within the pupil.

- Choose "Select" $\rightarrow$ "Modify" $\rightarrow$ "Expand". Enter the number of pixels by which to expand the selection - typically 50 pixels. This needs to be large enough to remove the hole in the selection caused by the infrared reflection.

- Choose "Select" $\rightarrow$ "Modify" $\rightarrow$ "Contract". Enter the number of pixels by which to contract the selection - this number must match the value used to expand the selection.

- Under the "Analysis" menu, choose "Record Measurements". This will start a running record of area measurements and file name.

- Close the image.

- Stop the action recording.

- Under the "File" menu, choose "Automate" and then "Batch". Then choose the Action to apply and browse for the file folder containing the images to analyze.

- When the batch has finished, select all recorded measurements and export the values as a text file.

- *Note: Photoshop allows a maximum of 700 recorded measurements at one time. Therefore, the images must be separated into groups of 700 prior to beginning batch processing. A short program can be written and applied to each folder of images to do this automatically. 


\section{REFERENCES}

Acland G, Aguirre G, Ray J, Zhang Q, Aleman T, Cideciyan A, Pearce-Kelling S, Anand V, Zeng Y, Maguire A, Jacobson S, Hauswirth W, Bennett J. 2001. Gene therapy restores vision in a canine model of childhood blindness. Nature genetics. 28(1):92-5.

Acland G, Ray K, Mellersh C, Langston A, Rine J, Ostrander E, Aguirre G. 1999. A novel retinal degeneration locus identified by linkage and comparative mapping of canine early retinal degeneration. Genomics. 59(2):134-42.

Awano T, Katz M, O’Brien D, Taylor J, Evans J, Khan S, Lobel P, Sohar I, Johnson G. 2006 a. A mutation in the cathepsin D gene (CTSD) in American Bulldogs with neuronal ceroid-lipofuscinosis. Molec. Genet. Metab. 87:341-8.

Awano T, Katz ML, O'Brien DP, Sohar I, Lobel P, Coates JR, Khan S, Johnson GC, Giger U, Johnson GS. 2006 b. A frame shift mutation in canine TPP1 ( the ortholog of human CLN2 ) in a juvenile Dachshund with neuronal ceroid lipofuscinosis. Molecular Genetics and Metabolism. 89:254-60.

Baehr W, Frederick JM. 2009. Naturally occurring animal models with outer retina phenotypes. Vision research. Elsevier Ltd; 49(22):2636-52.

Belenky M a, Smeraski C a, Provencio I, Sollars PJ, Pickard GE. 2003. Melanopsin retinal ganglion cells receive bipolar and amacrine cell synapses. The Journal of comparative neurology. 460(3):380-93.

Beutler E. 2004. Enzyme Replacement in Gaucher Disease. PLoS Medicine. 1(2):11821.

Bianco SR, Sun J, Fosmire SP, Hance K, Padilla ML, Ritt MG, Getzy DM, Duke RC, Withrow SJ, Lana S, Matthiesen DT, Dow SW, Bellgrau D, Cutter GR, Helfand SC, Modiano JF. 2003. Enhancing antimelanoma immune responses through apoptosis. Cancer gene therapy. 10(9):726-36.

Bond M, Holthaus S-MK, Tammen I, Tear G, Russell C. 2013. Use of model organisms for the study of neuronal ceroid lipofuscinosis. Biochimica et biophysica acta. Elsevier B.V.;

Bowmaker J, Dartnall H. 1980. Visual pigments of rods and cones in a human retina. Journal of Physiology. 298:501-11.

Bradley MM, Miccoli L, Escrig MA, Lang PJ. 2008. The pupil as a measure of emotional arousal and autonomic activation. Psychophysiology. 45(4):602-7. 
Bremner F. 2009. Pupil evaluation as a test for autonomic disorders. Clinical Autonomic Research. 19:88-101.

Brown TM, Lucas RJ. 2009. Melanopsin Phototransduction: Great Excitement over a Poor Catch. Current Biology. Elsevier Ltd; 19(6):R256-R257.

Bui B, Weisinger H, Sinclair A, Vingrys A. 1998. Comparison of guinea pig electroretinograms measured with bipolar corneal and unipolar intravitreal electrodes. Documenta ophthalmologica. 95(1):15-34.

Casal M, Haskins M. 2006. Large animal models and gene therapy. European journal of human genetics : EJHG. 14(3):266-72.

Chang M, Cooper JD, Sleat DE, Cheng SH, Dodge JC, Passini MA, Lobel P, Davidson BL. 2008. Intraventricular Enzyme Replacement Improves Disease Phenotypes in a Mouse Model of Late Infantile Neuronal Ceroid Lipofuscinosis. Molecular Therapy. 16(4):649-56.

Chauvet AE, Kesava PP, Goh CS, Badie B. 1998. Selective intraarterial gene delivery into a canine meningioma. Journal of neurosurgery. 88:870-3.

Dacey DM, Liao H, Peterson BB, Robinson FR, Smith VC, Pokorny J, Yau K, Gamlin PD. 2005 a. Melanopsin-expressing ganglion cells in primate retina signal colour and irradiance and project to the LGN. Nature. 433:749-54.

Dacey DM, Liao H, Peterson BB, Robinson FR, Smith VC, Pokorny J, Yau K, Gamlin PD. 2005 b. Melanopsin-expressing ganglion cells in primate retina signal colour and irradiance and project to the LGN. Nature. 433:749-54.

Dahlstrom A, Fuxe K, Hillarp N-A, Malmfors T. 1964. Adrenergic Mechanisms in the Pupillary Light-Reflex Path. Acta Physiologica Scandinavica. 62:119-24.

Daya S, Berns KI. 2008. Gene Therapy Using Adeno-Associated Virus Vectors. Society. 21(4):583-93.

Derwent JJK, Padnick-silver L, Mcripley M, Giuliano E, Linsenmeier RA, Narfstrom K. 2006. The Electroretinogram Components in Abyssinian Cats with Hereditary Retinal Degeneration. Investigative Ophthalmology. 47(8):3673-82.

Dyck J, Maze M, Haack C, Vuorilehto L, Shafer S. 1993. The Pharmacokinetics and Hemodynamic Effects of Intravenous and Intramuscular Dexmedetomidine Hydrochloride in Adult Human Volunteers. Anesthesiology. 78:813-20.

Ecker JL, Lall GS, Haq S, Altimus CM, Liao H, Gu AD, Barnard AR, Cahill H, Badea TC, Zhao H, Hankins MW, Berson DM, Lucas RJ, Yau K, Hattar S. 2008. Melanopsin cells are the principal conduits for rod - cone input to non-image-forming vision. Nature. 453(May):102-6. 
Fan X, Miles J, Takahashi N, Yao G. 2009 a. Abnormal transient pupillary light reflex in individuals with autism spectrum disorders. J Autism Dev Disord. 39:1499-508.

Fan X, Miles JH, Takahashi N, Yao G. 2009 b. Sex-Specific Lateralization of Contraction Anisocoria in Transient Pupillary Light Reflex. Invest Ophthalmol Vis Sci. 50(3):1137-44.

Farias FH, Zeng R Johnson GS, Wininger FA, Taylor JF, Schnabel RD, McKay SD, Lohi $\mathrm{H}$, Lindblad-Toh K, Wade CM, O'Brien DP KM. 2011. A truncating mutation in ATP13A2 is responsible for adult-onset neuronal ceroid lipofuscinosis in Tibetan Terriers.

Neurobiol. Dis. 42:468-74.

Felsburg PJ, Somberg RL, Hartnett BJ, Henthorn PS, Carding SR. 1998. Canine XLinked Severe Combined Immunodeficiency. Immunologic Research. 17:63-73.

Fotiou D, Stergiou V, Tsiptsios D, Lithari C, Nakou M, Karlovasitou A. 2009. Cholinergic deficiency in Alzheimer's and Parkinson's disease : Evaluation with pupillometry. Int J Psychophysiol. Elsevier B.V.; 73(2):143-9.

Fotiou F, Fountoulakis KN, Goulas A, Alexopoulos L, Palikaras A. 2000. Automated standardized pupillometry with optical method for purposes of clinical practice and research. Clinical physiology. 20(5):336-47.

Gamlin PDR, Mcdougal DH, Pokorny J, Smith VC, Yau K, Dacey DM. 2007. Human and macaque pupil responses driven by melanopsin-containing retinal ganglion cells. Vision Research. 47:946-54.

Green D, Kapousta-Bruneau N. 1999. A dissection of the electroretinogram from the isolated rat retina with microelectrode and drugs. Vis Neurosci. 16:727-41.

Grozdanic SD, Matic M, Sakaguchi DS, Kardon RH. 2007. Evaluation of Retinal Status Using Chromatic Pupil Light Reflex Activity in Healthy and Diseased Canine Eyes. Invest Ophthalmol Vis Sci. 48:5178-83.

Gurevich L, Slaughter M. 1993. Comparison of the waveforms of the ON bipolar neuron and the b-wave of the electroretinogram. Vision Res. 33:2431-5.

Haltia M. 2006. The neuronal ceroid-lipofuscinoses : From past to present. Neuropathology. 1762:850 - 856 .

Haltia M, Goebel HH. 2012. The neuronal ceroid-lipofuscinoses: A historical introduction. Biochimica et biophysica acta. Elsevier B.V.; 8-13.

Hargrave P, McDowell J. 1992. Rhodopsin and phototransduction. Int Rev Cytol. 137B:49-97. 
Hattar S, Lucas RJ, Mrosovsky N, Thompson S, Douglas RH, Hankins M, Lem J, Biel M, Hofmann F, Foster R, Yao K. 2003. Melanopsin and rod - cone photoreceptive systems account for all major accessory visual functions in mice. Nature. 424:76-81.

Hauswirth WW, Aleman TS, Kaushal S, Cideciyan A V, Schwartz SB, Wang L, Conlon TJ, Boye SL, Flotte TR, Byrne BJ, Jacobson SG. 2008. Treatment of leber congenital amaurosis due to RPE65 mutations by ocular subretinal injection of adeno-associated virus gene vector: short-term results of a phase I trial. Human gene therapy. 19(10):97990.

Herzog RW, Yang EY, Couto LB, Hagstrom JN, Elwell D, Fields P a, Burton M, Bellinger D a, Read MS, Brinkhous KM, Podsakoff GM, Nichols TC, Kurtzman GJ, High K a. 1999. Long-term correction of canine hemophilia $B$ by gene transfer of blood coagulation factor IX mediated by adeno-associated viral vector. Nature medicine. 5(1):56-63.

Hobert JA, Dawson G. 2006. Neuronal ceroid lipofuscinoses therapeutic strategies: Past, present and future. Biochimica et biophysica acta. 1762:945 - 953.

Kankipati L, Girkin C, Gamlin P. 2010. Post-illumination pupil response in subjects without ocular disease. Invest Ophthalmol Vis Sci. 51(5):2764-9.

Katz M, Coates J, Cooper J, O’Brien DP, Jeong M, Narfstrom K. 2008. Retinal Pathology in a Canine Model of Late Infantile Neuronal Ceroid Lipofuscinosis. Invest Ophthalmol Vis Sci. 49(6):2686-95.

Katz M, Coates J, Sibigtroth C, Taylor J, Carpentier M, Vuillemenot B, Kennedy D, O'Neill C. 2013. Enzyme Replacement Therapy Delays Disease Progression in a Canine Model of Late Infantile Neuronal Ceroid Lipofuscinosis. Submitted.

Katz M, Khan S, Awano T, Shahid A, Siakotos A, Johnson G. 2005. A mutation in the CLN8 gene in English Setter dogs with neuronal ceroid lipofuscinosis. Biochem.

Biophys. Res. Commun. 327:541-7.

Katz ML, Farias FH, Sanders DN, Zeng R, Khan S, Johnson GS, O’Brien DP. 2011. A missense mutation in canine CLN6 in an Australian shepherd with neuronal ceroid lipofuscinosis. Journal of biomedicine \& biotechnology. 2011:1-6.

Kyttälä A, Lahtinen U, Braulke T, Hofmann SL. 2006. Functional biology of the neuronal ceroid lipofuscinoses ( NCL ) proteins. Internal Medicine. 1762:920 - 933.

Lall GS, Revell VL, Momiji H, Enezi J Al, Altimus CM, Gu AD, Aguilar C, Cameron MA, Allender S, Hankins MW, Lucas RJ. 2010. Distinct Contributions of Rod, Cone, and Melanopsin Photoreceptors to Encoding Irradiance. Neuron. 417-28.

Larson M, Sessler D, McGuire J, Hynson J. 1991. Isoflurane, but not mild hypothermia, depresses the human pupillary light reflex. Anesthesiology. 75:62-7. 
Lin S, Shiu W, Liu P, Cheng F, Lin Y, Wang W. 2009. The effects of different anesthetic agents on short electroretinography protocol in dogs. The Journal of Veterinary Medical Science. 71:763-8.

Lowenstein O, Lowenfeld IE. 1950. Mutual Role of Sympathetic and Parasympathetic in Shaping of the Pupillary Reflex to Light. Archives of Neurology and Psychiatry. 64(3):341-77.

Lucas RJ, Hattar S, Takao M, Berson DM. 2003. Diminished Pupillary Light Reflex at High Irradiances in Melanopsin-Knockout Mice. Science. 299:245-7.

Markwell E, Feigl B, Zele A. 2010. Intrinsically photosensitive melanopsin retinal ganglion cell contributions to the pupillary light reflex and circadian rhythm. Clinical and Experimental Optometry. 93(May):137-49.

Marmor MF, Holder GE, Seeliger MW, Yamamoto S. 2004. Standard for clinical electroretinography (2004 update). Documenta Ophthalmologica. 108(2):107-14.

Melillo P, Pecchia L, Testa F, Rossi S, Bennett J, Simonelli F. 2012. Pupillometric analysis for assessment of gene therapy in Leber Congenital Amaurosis patients. Biomedical engineering online. 11:40.

Mole S, Williams R, Goebel H. 2011. The Neuronal Ceroid Lipofuscinoses (Batten Disease). 2nd ed. London: Oxford University Press;

Narfström K, Ekesten B, Rosolen SG, Spiess BM, Percicot CL, Ofri R. 2002. Guidelines for clinical electroretinography in the dog. Documenta Ophthalmologica. 105(2):83-92.

Narfstrom K, Katz M, Ford M, Redmond T, Rakoczy E, Bragadottir R. 2003 a. In Vivo Gene Therapy in Young and Adult RPE65-/- Dogs Produces Long-Term Visual Improvement. Journal of Heredity. 94(1):31-7.

Narfstrom K, Katz ML, Bragadottir R, Seeliger M, Boulanger A, Redmond TM, Caro L, Lai C, Rakoczy PE. 2003 b. Functional and Structural Recovery of the Retina after Gene Therapy in the RPE65 Null Mutation Dog. Invest Ophthalmol Vis Sci. 44(4):1663-72.

Neitz J, Geist T, Jacobs GH. 1989. Color vision in the dog. Visual Neuroscience. 3:11925.

O'Brien D, Katz M. 2008. Neuronal ceroid lipofuscinosis in three Australian shepherd littermates. J. Vet. Internal Med. 22:472-5.

Ostrander E a, Giniger E. 1997. Semper fidelis: what man's best friend can teach us about human biology and disease. American journal of human genetics. 61(3):475-80. 
Palmer D, Tammen I, Drogemuller C, Johnson G, Katz M, Lingaas F. 2011. Large animal models of neuronal ceroid lipofuscinoses. In: Mole S, Williams R, Goebel H, editors. The Neuronal Ceroid Lipofuscinoses (Batten Disease). 2nd ed. London: Oxford University Press; p. 284-320.

Park JC, Moura AL, Raza AS, Rhee DW, Kardon RH, Hood DC. 2011. Toward a Clinical Protocol for Assessing Rod, Cone, and Melanopsin Contributions to the Human Pupil Response. Invest Ophthalmol Vis Sci. 52:6624-35.

Pearce J, Whiting R, Castaner L, Narfström K, Katz M. 2012. Multifocal Retinopathy in A Colony of Miniature Longhaired Dachshunds with Late Infantile Neuronal Ceroid Lipofuscinosis. 43rd Annual Meeting of the American College of Veterinary Ophthalmologists.

Penn R, Hagins W. 1969. Signal transmission along retinal rods and the origin of the electroretinographic a-wave. Nature. 223:201-5.

Pierret C, Morrison JA, Kirk MD. 2008. Treatment of lysosomal storage disorders : Focus on the neuronal ceroid-lipofuscinoses. Acta Neurobiologiae Experimentalis (Warsaw). 429-42.

Ray J, Bouvet A, DeSanto C, Fyfe JC, Xu D, Wolfe JH, Aguirre GD, Patterson DF, Haskins ME, Henthorn PS. 1998. Cloning of the canine beta-glucuronidase cDNA, mutation identification in canine MPS VII, and retroviral vector-mediated correction of MPS VII cells. Genomics. 48(2):248-53.

Roorda a, Williams DR. 1999. The arrangement of the three cone classes in the living human eye. Nature. 397(6719):520-2.

Sanders DN, Farias FH, Johnson GS, Chiang V, Cook JR, O'Brien DP, Hofmann SL, Lu $\mathrm{J}-\mathrm{Y}$, Katz ML. 2010. A mutation in canine PPT1 causes early onset neuronal ceroid lipofuscinosis in a Dachshund. Molecular genetics and metabolism. 100(4):349-56.

Sargan DR. 2004. IDID: inherited diseases in dogs: web-based information for canine inherited disease genetics. Mammalian genome: official journal of the International Mammalian Genome Society. 15(6):503-6.

Scagliotti RH. 1999. Comparative Neuro-ophthalmology. In: Gellat KN, editor. Veterinary Ophthalmology. 3rd ed. Gainesville, FL: Lippincott Williams \& Wilkins; p. 1307-400.

De Seze J, Arndt C, Stojkovic T, Ayachi M, Gauvrit JY, Bughin M, Michel T, Pruvo J, Hache J, Vermersch P. 2001. Pupillary disturbances in multiple sclerosis : correlation with MRI findings. J Neurol Sci. 188:37-41.

Sharpe L, Pickworth W. 1981. Pharmacologic evidence for a tonic muscarinic inhibitory input to the Edinger-Westphal nucleus in the dog. Experimental Neurology. 71:176-90. 
Sohar, I., Sleat, D. E., Jadot, M. and Lobel P. 1999. Biochemical characterization of a lysosomal protease deficient in classical late infantile neuronal ceroid lipofuscnosis (LINCL) and development of an enzyme-based assay for diagnosis and exclusion of LINCL in human specimens and animal models. J. Neurochem. 73:700-11.

Sondhi D, Hackett NR, Peterson D a, Stratton J, Baad M, Travis KM, Wilson JM, Crystal RG. 2007. Enhanced survival of the LINCL mouse following CLN2 gene transfer using the rh.10 rhesus macaque-derived adeno-associated virus vector. Molecular therapy: the journal of the American Society of Gene Therapy. 15(3):481-91.

Souweidane M, Fraser J, Arkin L, Sondhi D, Hackett N, Kaminsky S, Heier L, Kosofsky B, Worgall S, Crystal R, Kaplitt M. 2010. Gene therapy for late infantile neuronal ceroid lipofuscinosis: neurosurgical considerations. Pediatrics. 6(August):115-22.

Steinhauer SR, Siegle GJ, Condray R, Pless M. 2004. Sympathetic and parasympathetic innervation of pupillary dilation during sustained processing. International journal of psychophysiology : official journal of the International Organization of Psychophysiology. 52(1):77-86.

Stockton R, Slaughter M. 1989. B-wave of the electroretinogram. A reflection of ON bipolar cell activity. J Gen Physiol. 93:101-22.

Tayefeh F, Larson MD, Sessler DI, Eger El, Bowland T. 1997. Time-dependent changes in heart rate and pupil size during desflurane or sevoflurane anesthesia. Anesthesia and Analgesia. 85(6):1362-6.

Thompson S, Stasheff SF, Hernandez J, Nylen E, East JS, Kardon RH, Pinto LH, Mullins RF, Stone EM. 2011. Different inner retinal pathways mediate rod-cone input in irradiance detection for the pupillary light reflex and regulation of behavioral state in mice. Investigative ophthalmology \& visual science. 52(1):618-23.

Thompson S, Whiting REH, Kardon RH, Stone EM. 2010. Effects of hereditary retinal degeneration due to a CEP290 mutation on the feline pupillary light reflex. Veterinary Ophthalmology. 151-7.

Tomkinson B. 1999. Tripeptidyl peptidases : enzymes that count. Trends in biochemical sciences. 24(September):1210-3.

Veske A, Nilsson SE, Narfström K, Gal A. 1999. Retinal Dystrophy of Swedish Briard/Briard-Beagle Dogs is Due to a 4-bp Deletion in RPE65. Genomics. 57(1):57-61.

Vuillemenot BR, Katz ML, Coates JR, Kennedy D, Tiger P, Kanazono S, Lobel P, Sohar I, Xu S, Cahayag R, Keve S, Koren E, Bunting S, Tsuruda LS, O'Neill C a. 2011. Intrathecal tripeptidyl-peptidase 1 reduces lysosomal storage in a canine model of late infantile neuronal ceroid lipofuscinosis. Molecular genetics and metabolism. 104(3):32537. 
Vuillemenot BR, Kennedy D, Katz M, Al. E. 2013. Safety, pharmacokinetics, and CNS distribution of recombinant human tripeptidyl peptidase-1 administered to the CSF in a canine model of classical late infantile neuronal ceroid lipofuscinosis. Submitted.

Whiting RE, Yao G, Narfström K, Pearce JW, Coates JR, Dodam JR, Castaner LJ, Katz ML. 2013. Quantitative Assessment of the Canine Pupillary Light Reflex. Submitted.

Wilhelm H. 2008. The pupil. Current Opinion in Neurology. 21:36-42.

Worgall S, Sondhi D, Hackett NR, Kosofsky B, Kekatpure M V, Neyzi N, Dyke JP, Ballon D, Heier L, Greenwald BM, Christos P, Mazumdar M, Souweidane MM, Kaplitt MG, Crystal RG. 2008. Treatment of Late Infantile Neuronal Ceroid Lipofuscinosis by CNS Administration of a Serotype 2 Adeno-Associated Virus Expressing CLN2 cDNA. Human Gene Therapy. 19(May):1-12.

Xu S, Sleat DE, Jadot M, Lobel P. 2010. Glial fibrillary acidic protein is elevated in the lysosomal storage disease classical late-infantile neuronal ceroid lipofuscinosis but is not a component of the storage material. Biochem J. 428(3):355-62.

Yao G, Zhang K, Bellassai M, Chang B, Lei B. 2006. Ultraviolet Light - Induced and Green Light - Induced Transient Pupillary Light Reflex in Mice. Current Eye Research. 31:925-33.

Yau K-W. 1994. Phototransduction mechanisms in retinal rods and cones. Invest Ophthal Vis Sci. 35:9-32.

Yoshitomi T, Ito Y. 1986. Double reciprocal innervations in dog iris sphincter and dilator muscles. Investigative ophthalmology \& visual science. 27(1):83-91.

Yoshitomi T, Ito Y, Inomata H. 1985. Adrenergic excitatory and cholinergic inhibitory innervations in the human iris dilator. Experimental Eye Research. 40:453-9. 


\section{VITA}

Rebecca E. H. Whiting was born as Rebecca Elizabeth Hannah on November 6, 1983 in Kansas City, Missouri. She earned a B.S. in Biomedical Engineering in May, 2006 from Illinois Institute of Technology in Chicago, Illinois. She entered the master's program for Biological Engineering at the University of Missouri in the Summer of 2007 and transferred to the doctorate program in the Fall of 2008. She studied hereditary retinal degeneration in an Abyssinian cat model under Dr. Kristina Narfström until the end of 2009 , when Dr. Narfstrom retired. She then began the study of the pupillary light reflex in a canine model of neuronal ceroid lipofuscinosis under Dr. Martin L. Katz. 